hep-th/

\title{
Gauging Spacetime Symmetries On The Worldsheet And The Geometric Langlands Program
}

\author{
Meng-Chwan Tan* \\ School of Natural Sciences, Institute for Advanced Study \\ Princeton, New Jersey 08540, USA \\ e-mail: tan@ias.edu
}

\begin{abstract}
We study the two-dimensional twisted $(0,2)$ sigma-model on various smooth complex flag manifolds $G / B$, and explore its relevance to the geometric Langlands program. We find that an equivalence - at the level of the holomorphic chiral algebra - between a bosonic string on $G / B$ and a $B$-gauged version of itself on $G$, will imply an isomorphism of classical $\mathcal{W}$-algebras and a level relation which underlie a geometric Langlands correspondence for $G=S L(N, \mathbf{C})$. This furnishes an alternative physical interpretation of the geometric Langlands correspondence for $G=S L(N, \mathbf{C})$, to that demonstrated earlier by Kapustin and Witten via an electric-magnetic duality of four-dimensional gauge theory. Likewise, the Hecke operators and Hecke eigensheaves will have an alternative physical interpretation in terms of the correlation functions of local operators in the holomorphic chiral algebra of a quasi-topological sigma-model without boundaries. A forthcoming paper will investigate the interpretation of a "quantum" geometric Langlands correspondence for $G=S L(N, \mathbf{C})$ in a similar setting, albeit with fluxes of the sigma-model moduli which induce a "quantum" deformation of the relevant classical algebras turned on.
\end{abstract}

\footnotetext{
* On leave of absence from the National University of Singapore.
} 


\section{Introduction}

The geometric Langlands correspondence has recently been given an elegant physical interpretation by Kapustin and Witten in their seminal paper [1] - by considering a certain twisted $\mathcal{N}=4$ supersymmetric Yang-Mills theory in four-dimensions compactified on a complex Riemann surface $C$, the geometric Langlands correspondence associated to a holomorphic $G$-bundle on $C$ can be shown to arise naturally from an electric-magnetic duality in four-dimensions. To be more specific, it can be shown [1] that one can, for example, relate various mathematical objects and concepts of the correspondence such as Hecke eigensheaves and the action of the Hecke operator, to the boundaries and the 't Hooft line operator of the underlying four-dimensional quantum gauge theory. Through a four-dimensional electric-magnetic duality, or a mirror symmetry of the resulting two-dimensional topological sigma-model at low-energies, one can then map the relevant objects on either side of the correspondence to their corresponding partners on the other side, thus furnishing a purely physical interpretation of the geometric Langlands conjecture.

The work of Kapustin and Witten centres around a gauge-theoretic interpretation of the geometric Langlands correspondence. However, it does not shed any light on the utility of two-dimensional axiomatic conformal field theory in the geometric Langlands program, which, incidentally, is ubiquitous in the mathematical literature on the subject [2, 3, 4, 5, 6]. This seems rather puzzling. Afterall, the various axiomatic definitions of a conformal field theory that fill the mathematical literature, are based on established physical concepts, and it is therefore natural to expect that in any physical interpretation of the geometric Langlands correspondence, a two-dimensional conformal field theory of some sort will be involved. It will certainly be illuminating for the geometric Langlands program as a whole, if one can deduce the conformal field-theoretic approach developed in the mathematical literature, from the gauge-theoretic approach of Kapustin and Witten, or vice-versa.

Note that the gauge-theoretic approach to the program necessarily involves a certain two-dimensional quantum field theory in its formulation, a generalised topological sigmamodel to be exact. This strongly suggests that perhaps a good starting point towards elucidating the connection between the conformal field-theoretic and gauge-theoretic approaches, would be to explore other physical models in two-dimensions which will enable us to make direct contact with the central results of the correspondence derived from the axiomatic conformal field-theoretic approach. The work in this paper represents our modest attempt towards this aim.

The key ingredients in the conformal field-theoretic approach to the geometric Lang- 
lands correspondence are, affine Lie algebras at the critical level without stress tensors [7], and $\mathcal{W}$-algebras (defined by a Drinfeld-Sokolov or DS reduction procedure) associated to the affine versions of the Langlands dual of the Lie algebras [7, 8]. The duality between classical $\mathcal{W}$-algebras which underlies the conformal field-theoretic approach to the correspondence, is just an isomorphism between the Poisson algebra generated by the centre $\mathfrak{z}(\widehat{\mathfrak{g}})$ of the completed universal enveloping algebra of the affine Lie algebra $\widehat{\mathfrak{g}}$ at the critical level, where $\mathfrak{g}$ is the simple Lie algebra of the group $G$, and the classical $\mathcal{W}$-algebra associated to the affine Lie algebra ${ }^{L} \widehat{\mathfrak{g}}$ in the limit of large level $k^{\prime}-\mathcal{W}_{\infty}\left({ }^{L} \widehat{\mathfrak{g}}\right)$, where ${ }^{L} \mathfrak{g}$ is the simple Lie algebra of the Langlands dual group ${ }^{L} G$; in other words, a geometric Langlands correspondence for $G$ simply originates from an isomorphism $\mathfrak{z}(\widehat{\mathfrak{g}}) \simeq \mathcal{W}_{\infty}\left({ }^{L} \widehat{\mathfrak{g}}\right)$ of Poisson algebras [6, 9]. This statement is accompanied by a relation $\left(k+h^{\vee}\right) r^{\vee}=\left(k^{\prime}+{ }^{L} h^{\vee}\right)^{-1}$ between the generic levels $k$ and $k^{\prime}$ of $\widehat{\mathfrak{g}}$ and ${ }^{L} \widehat{\mathfrak{g}}$ respectively (where $r^{\vee}$ is the lacing number of $\mathfrak{g}$, and $h^{\vee}$ and ${ }^{L} h^{\vee}$ are the dual Coxeter numbers of $\mathfrak{g}$ and ${ }^{L} \mathfrak{g}$ respectively), which defines a "quantum" generalisation of the above isomorphism of classical $\mathcal{W}$-algebras [6, 9], whereby the $k=-h^{\vee}$ and $k^{\prime}=\infty$ limits just correspond to the classical isomorphism mentioned herein that we shall be discussing in this paper.

A strong hint that one should be considering for our purpose a two-dimensional twisted $(0,2)$ sigma-model on a flag manifold, stems from our recent understanding of the role sheaves of "Chiral Differential Operators" (or CDO's) play in the description of its holomorphic chiral algebra [10], and from the fact that global sections of CDO's on a flag manifold furnish a module of an affine Lie algebra at the critical level [10, 11]. On the other hand, since Toda field theories lead to free-field realisations of the $\mathcal{W}$-algebras defined by the DS reduction scheme mentioned above (see Sect. 6 of [12], and the references therein), and since the Toda theory can be obtained as a gauge-invariant content of a certain gauged WZW theory [13, 14], it ought to be true that by relating a relevant aspect of the sigma-model on a flag manifold to a gauged WZW model, one should be able to uncover a physical manifestation of the isomorphism of (classical) $\mathcal{W}$-algebras. Indeed, we shall show that an equivalence at the level of the holomorphic chiral algebra - between a bosonic string on a smooth flag manifold $G / B$ and a $B$-gauged WZW model on $G$, where $G=S L(N, \mathbf{C})$, will necessarily imply an isomorphism of classical $\mathcal{W}$-algebras and the relation $\left(k+h^{\vee}\right) r^{\vee}=\left(k^{\prime}+{ }^{L} h^{\vee}\right)^{-1}$ which underlie a geometric Langlands correspondence for $G=S L(N, \mathbf{C})$. Since a string on a group manifold $G$ can be expressed as a WZW model on $G$ [15], it would mean that an equivalence, at the level of the holomorphic chiral algebra, between a bosonic string on a smooth coset manifold $G / B$ and a $B$-gauged version of itself on $G$ - a statement which 
stems from the ubiquitous notion that one can always physically interpret a geometrical symmetry of the target space as a gauge symmetry in the worldsheet theory - will imply a geometric Langlands correspondence for $G=S L(N, \mathbf{C})$. This furnishes an alternative physical interpretation of the geometric Langlands correspondence for $G=S L(N, \mathbf{C})$ to that of an electric-magnetic duality of four-dimensional gauge theory. Likewise, the Hecke operators and Hecke eigensheaves will also lend themselves to different physical interpretations - instead of line operators and branes in a two-dimensional topological sigma-model, they are, in our case, associated to the correlation functions of local operators that span the holomorphic chiral algebra of a quasi-topological sigma-model without boundaries. Our results therefore open up a new way of looking at the correspondence, thus providing the prospect of novel mathematical and physical insights for the program in general.

\section{A Brief Summary and Plan of the Paper}

We shall now give a brief summary and plan of the paper.

In $\S 2$, we shall show that an equivalence - at the level of the holomorphic chiral algebra - between a bosonic string on $G / B$ and a $B$-gauged version of itself on $G$, will necessarily imply a geometric Langlands correspondence for $G=S L(N, \mathbf{C})$, where $N=2,3$. We begin by considering the twisted $(0,2)$ sigma-model on a complex flag manifold defined by the coset space $S L(N) / B$ where $N=2,3$, and $B$ is a Borel subgroup containing upper triangular matrices of $S L(N) 1$ We then explain how a subspace of the global sections of the sheaf of CDO's, describing the holomorphic chiral subalgebra of the sigma-model on $S L(N) / B$, will furnish a module of an affine $S L(N)$ algebra at the critical level. This in turn will allow us to show, using the results in appendix $\mathrm{A}$, that the classical centre of the completed universal enveloping algebra of the affine $S L(N)$ algebra at the critical level $-\mathfrak{z}\left(\widehat{\mathfrak{s l}}_{N}\right)$, is spanned by the Laurent modes of certain local fields of spins 2 and 3 in the classical holomorphic chiral algebra of the purely bosonic sector of the sigma-model on $S L(N) / B$. Next, we turn to a dual description of this classical, holomorphic chiral algebra in the purely bosonic sector (or bosonic string part) of the sigma-model on $S L(N) / B$ - the classical, holomorphic BRST-cohomology (or chiral algebra) of a B-gauged WZW model on $S L(N)$. One can then show that an equivalence between these classical, holomorphic chiral algebras will necessarily imply an isomorphism $\mathfrak{z}(\widehat{\mathfrak{g}}) \simeq \mathcal{W}_{\infty}\left({ }^{L} \widehat{\mathfrak{g}}\right)$ of Poisson algebras and the level relation $\left(k+h^{\vee}\right) r^{\vee}=\left(k^{\prime}+{ }^{L} h^{\vee}\right)^{-1}$ that underlie a geometric Langlands correspondence for $G=S L(N)$, where $N=2,3$.

In $\S 3$, we will generalise our arguments in $\S 2$ to arbitrary $N$. To this end, we will first

\footnotetext{
${ }^{1}$ Here and henceforth, in writing $S L(N)$, we really mean $S L(N, \mathbf{C})$.
} 
discuss the twisted sigma-model on any complex flag manifold $S L(N) / B$, and the global sections of the sheaf of CDO's on $S L(N) / B$ associated to the chiral algebra of the purely bosonic sector of the sigma-model that will furnish a module of an affine $S L(N)$ algebra at the critical level. We will then proceed to discuss the construction of higher-spin analogs of the Segal-Sugawara tensor from the affine $S L(N)$ algebra, and show that these fields of higher spins which are in the classical holomorphic chiral algebra of the purely bosonic sector of the sigma-model on $S L(N) / B$, will have Laurent modes that span the classical centre $\mathfrak{z}\left(\widehat{\mathfrak{s l}}_{N}\right)$ of the completed universal enveloping algebra of the affine $S L(N)$ algebra at critical level. Next, we will outline the mathematical Drinfeld-Sokolov reduction procedure in [7] of defining $\mathcal{W}_{k^{\prime}}(\widehat{\mathfrak{g}})$, a $\mathcal{W}$-algebra associated to $\widehat{\mathfrak{g}}$ at level $k^{\prime}$, via a Hecke algebra. Thereafter, we will show that the holomorphic sector of the BRST-cohomology of the B-gauged WZW model on $S L(N)$ physically realises, in all generality, this particular Hecke algebra, i.e., the holomorphic BRST-cohomology of the $B$-gauged WZW model on $S L(N)$ always consist of local operators which generate a $\mathcal{W}_{k^{\prime}}\left(\widehat{\mathfrak{s l}}_{N}\right)$ OPE algebra. Hence, its classical, holomorphic BRST-cohomology will always consist of local fields with Laurent modes that generate a classical $\mathcal{W}_{\infty}\left(\widehat{\mathfrak{s l}}_{N}\right)$-algebra. By specialising our analysis (in the classical limit) to $N=2,3$, we will make contact with the results in $\S 2$. One can now extend the arguments in $\S 2$ for $N=2,3$ to any $N$; since $\mathfrak{g}=\mathfrak{s l}_{N}={ }^{L} \mathfrak{g}$, an equivalence - at the level of the classical holomorphic chiral algebra - between the purely bosonic sector (or bosonic string part) of the sigma-model on $S L(N) / B$ and the $B$-gauged WZW model on $S L(N)$, will necessarily imply an isomorphism $\mathfrak{z}(\widehat{\mathfrak{g}}) \simeq \mathcal{W}_{\infty}\left({ }^{L} \widehat{\mathfrak{g}}\right)$ of Poisson algebras and the level relation $\left(k+h^{\vee}\right) r^{\vee}=\left(k^{\prime}+{ }^{L} h^{\vee}\right)^{-1}$ which underlie a geometric Langlands correspondence for $G=S L(N)$. That is, an equivalence - at the level of the holomorphic chiral algebra - between a bosonic string on $G / B$ and a $B$-gauged version of itself on $G$, will necessarily imply a geometric Langlands correspondence for any $G=S L(N, \mathbf{C})$.

In $\S 4$, we shall derive, via the isomorphism of classical $\mathcal{W}$-algebras discussed in $\S 3$, a correspondence between flat holomorphic ${ }^{L} G$-bundles on the worldsheet $\Sigma$ and Hecke eigensheaves on the moduli space $\operatorname{Bun}_{G}$ of holomorphic $G$-bundles on $\Sigma$, where $G=S L(N)$. Lastly, we shall physically interpret the Hecke eigensheaves and Hecke operators of the geometric Langlands program in terms of the correlation functions of purely bosonic local operators in the holomorphic chiral algebra of the twisted $(0,2)$ sigma-model on the complex flag manifold $S L(N) / B$.

In appendix $\mathrm{A}$, we will review the two-dimensional twisted $(0,2)$ sigma-model considered in [10], and explain its relation to the theory of CDO's. In particular, we will describe how 
the relevant physical features of the sigma-model and its holomorphic chiral algebra, can be interpreted in terms of the sheaf of CDO's and its Cech-cohomology.

Relation to the Gauge-Theoretic Approach

Though we have not made any explicit connections to the gauge-theoretic approach of Kapustin and Witten yet, we hope to be able to address this important issue in a later publication, perhaps with the insights gained in this paper.

\section{A "Quantum" Geometric Langlands Correspondence}

A forthcoming paper will investigate the interpretation of a "quantum" geometric Langlands correspondence for $G=S L(N)$ in a similar physical context, albeit with fluxes of the sigma-model moduli turned on, such that the level of the affine $S L(N)$ algebra with a module furnished by the global sections of the sheaf CDO's on $X=S L(N) / B$, can be deformed away from the critical value, whereby a "quantum" deformation of our present setup can be defined.

\section{An Equivalence of Classical Holomorphic Chiral Algebras and the Geometric Langlands Correspondence for $G=S L(2)$ and $S L(3)$}

In this section, we shall study explicit examples of the twisted sigma-model on the complex flag manifolds $S L(2) / B$ and $S L(3) / B$, and the corresponding sheaves of CDO's that describe its holomorphic chiral algebra. We shall also study the holomorphic BRSTcohomology of a $B$-gauged WZW model on $S L(2)$ and $S L(3)$. We will then show that an equivalence - at the level of the holomorphic chiral algebra - between a bosonic string on $S L(N) / B$ and a $B$-gauged version of itself on $S L(N)$, where $N=2,3$, will imply an isomorphism of classical $\mathcal{W}$-algebras and the relation $\left(k+h^{\vee}\right) r^{\vee}=\left(k^{\prime}+h^{\vee}\right)^{-1}$ which underlie a geometric Langlands correspondence for $G=S L(2)$ and $S L(3)$ respectively.

\subsection{The Twisted Sigma-Model on $S L(2) / B$ and its Classical Holomorphic Chiral Algebra}

Let us take $X=S L(2) / B$. In other words, since $S L(2) / B \cong \mathbb{C P}^{1}$, we will be exploring and analysing the chiral algebra $\mathcal{A}$ of operators in the twisted $(0,2)$ model on $\mathbb{C P}^{1}$. To this end, we will work locally on the worldsheet $\Sigma$, choosing a local complex parameter $z$.

Now, $\mathbb{C P}^{1}$ can be regarded as the complex $\gamma$-plane plus a point at infinity. Thus, we can cover it by two open sets, $U_{1}$ and $U_{2}$, where $U_{1}$ is the complex $\gamma$-plane, and $U_{2}$ is the complex $\tilde{\gamma}$-plane, where $\tilde{\gamma}=1 / \gamma$. 
Since $U_{1}$ is isomorphic to $\mathbb{C}$, the sheaf of CDO's in $U_{1}$ can be described by a single free $\beta \gamma$ system with action

$$
I=\frac{1}{2 \pi} \int\left|d^{2} z\right| \beta \partial_{\bar{z}} \gamma
$$

Here $\beta$ and $\gamma$, are fields of dimension $(1,0)$ and $(0,0)$ respectively. They obey the usual free-field OPE's; there are no singularities in the operator products $\beta(z) \cdot \beta\left(z^{\prime}\right)$ and $\gamma(z) \cdot \gamma\left(z^{\prime}\right)$, while

$$
\beta(z) \gamma\left(z^{\prime}\right) \sim-\frac{1}{z-z^{\prime}}
$$

Similarly, the sheaf of CDO's in $U_{2}$ is described by a single free $\tilde{\beta} \tilde{\gamma}$ system with action

$$
I=\frac{1}{2 \pi} \int\left|d^{2} z\right| \tilde{\beta} \partial_{\bar{z}} \tilde{\gamma}
$$

where the fields $\tilde{\beta}$, and $\tilde{\gamma}$ obey the same OPE's as $\beta$ and $\gamma$. In other words, the non-trivial OPE's are given by

$$
\tilde{\beta}(z) \tilde{\gamma}\left(z^{\prime}\right) \sim-\frac{1}{z-z^{\prime}} .
$$

In order to describe a globally-defined sheaf of CDO's, one will need to glue the free conformal field theories with actions (2.1) and (2.3) in the overlap region $U_{1} \cap U_{2}$. To do so, one must use the admissible automorphisms of the free conformal field theories defined in (A.29) - A.30) to glue the free-fields together. In the case of $X=\mathbb{C P}^{1}$, the automorphisms will be given by

$$
\begin{aligned}
& \tilde{\gamma}=\frac{1}{\gamma} \\
& \tilde{\beta}=-\gamma^{2} \beta+2 \partial_{z} \gamma .
\end{aligned}
$$

As there is no obstruction to this gluing in the twisted sigma-model on any flag manifold $S L(N) / B$ [11], a sheaf of CDO's can be globally-defined on the $\mathbb{C P}^{1}$ target-space.

Global Sections of the Sheaf of CDO's on $X=S L(2) / B$

Recall that for a general manifold $X$ of complex dimension $n$, the chiral algebra $\mathcal{A}$ will be given by $\mathcal{A}=\bigoplus_{g_{R}=0}^{g_{R}=n} H^{g_{R}}\left(X, \widehat{\mathcal{O}}_{X}^{c h}\right)$ as a vector space. Since $\mathbb{C P}^{1}$ has complex dimension 1, we will have, for $X=\mathbb{C P}^{1}$, the relation $\mathcal{A}=\bigoplus_{g_{R}=0}^{g_{R}=1} H^{g_{R}}\left(\mathbb{C P}^{1}, \widehat{\mathcal{O}}_{\mathbb{P}^{1}}^{c h}\right)$. Thus, in order to understand the chiral algebra of the twisted sigma-model, one needs only to study the global sections of the sheaf $\widehat{\mathcal{O}}_{\mathbb{P}^{1}}^{c h}$, and its first Cech cohomology $H^{1}\left(\mathbb{C P}^{1}, \widehat{\mathcal{O}}_{\mathbb{P}^{1}}^{c h}\right)$. However, for our purpose, it would suffice to study just the purely bosonic sector of $\mathcal{A}$ - from our $\bar{Q}_{+}$-Cech cohomology dictionary, this translates to studying the global sections $H^{0}\left(\mathbb{C P}^{1}, \widehat{\mathcal{O}}_{\mathbb{P}^{1}}^{c h}\right)$ only. 
At dimension 0 , the space of global sections $H^{0}\left(\mathbb{C P}^{1}, \widehat{\mathcal{O}}_{\mathbb{P}^{1} ; 0}^{c h}\right)$ must be spanned by functions of arbitrary degree in $\gamma$. Since all regular, holomorphic functions on a compact Riemann surface such as $\mathbb{C P}^{1}$ must be constants, we find that the space of global sections at dimension 0 , given by $H^{0}\left(\mathbb{C P}^{1}, \widehat{\mathcal{O}}_{\mathbb{P}^{1} ; 0}^{c h}\right)$, is one-dimensional and generated by 1 .

Let us now ascertain the space $H^{0}\left(\mathbb{C P}^{1}, \widehat{\mathcal{O}}_{\mathbb{P}^{1} ; 1}^{c h}\right)$ of global sections of dimension 1 . In order to get a global section of $\widehat{\mathcal{O}}_{\mathbb{P}^{1}}^{c h}$ of dimension 1 , we can act on a global section of $\widehat{\mathcal{O}}_{\mathbb{P}^{1}}^{c h}$ of dimension 0 with the partial derivative $\partial_{z}$. Since $\partial_{z} 1=0$, this prescription will not apply here.

One could also consider operators of the form $f(\gamma) \partial_{z} \gamma$, where $f(\gamma)$ is a holomorphic function of $\gamma$. However, there are no such global sections either - such an operator, by virtue of the way it transforms purely geometrically under (2.5), would correspond to a section of $\Omega^{1}\left(\mathbb{C P}^{1}\right)$, the sheaf of holomorphic differential forms $f(\gamma) d \gamma$ on $\mathbb{C P}^{1}$, and from the classical result $H^{0}\left(\mathbb{C P}^{1}, \Omega^{1}\left(\mathbb{C P}^{1}\right)\right)=0$, which continues to hold in the quantum theory, we see that $f(\gamma) \partial_{z} \gamma$ cannot be a dimension 1 global section of $\widehat{\mathcal{O}}_{\mathbb{P}^{1}}^{c h}$.

Other possibilities include operators which are linear in $\beta$. In fact, from the automorphism relation of (2.6), we find an immediate example as the LHS, $\tilde{\beta}$, is by definition regular in $U_{2}$, while the RHS, being polynomial in $\gamma, \partial_{z} \gamma$ and $\beta$, is manifestly regular in $U_{1}$. Their being equal means that they represent a dimension 1 global section of $\widehat{\mathcal{O}}_{\mathbb{P}^{1}}^{c h}$ that we will call $J_{+}$:

$$
J_{+}=-\gamma^{2} \beta+2 \partial_{z} \gamma=\tilde{\beta} .
$$

The construction is completely symmetric between $U_{1}$ and $U_{2}$, with $\gamma \leftrightarrow \tilde{\gamma}, \beta \leftrightarrow \tilde{\beta}$, so a reciprocal formula gives another dimension 1 global section $J_{-}$:

$$
J_{-}=\beta=-\tilde{\gamma}^{2} \tilde{\beta}+2 \partial_{z} \tilde{\gamma} .
$$

Hence, $J_{+}$and $J_{-}$give us two dimension 1 global sections of the sheaf $\widehat{\mathcal{O}}_{\mathbb{P}^{1}}^{c h}$. Since these are global sections of a sheaf of chiral vertex operators, we can construct more of them from their OPE's. There are no singularities in the $J_{+} \cdot J_{+}$or $J_{-} \cdot J_{-}$operator products, but

$$
J_{+} J_{-} \sim \frac{2 J_{3}}{z-z^{\prime}}-\frac{2}{\left(z-z^{\prime}\right)^{2}},
$$

where $J_{3}$ is another global section of dimension 1 given by

$$
J_{3}=-\gamma \beta \text {. }
$$


(Note that normal-ordering is again understood for all operators above and below).

Notice that since $\left\{J_{+}, J_{-}, J_{3}\right\}$ are $\psi^{\bar{i}}$-independent, they are purely bosonic operators that belong in $H^{0}\left(\mathbb{C P}^{1}, \widehat{\mathcal{O}}_{\mathbb{P}^{1} ; 1}^{c h}\right)$. One can verify that they satisfy the following closed OPE algebra:

$$
\begin{aligned}
J_{3}(z) J_{+}\left(z^{\prime}\right) & \sim \frac{+J_{+}\left(z^{\prime}\right)}{z-z^{\prime}}, \\
J_{3}(z) J_{-}\left(z^{\prime}\right) & \sim \frac{-J_{-}\left(z^{\prime}\right)}{z-z^{\prime}}, \\
J_{3}(z) J_{3}\left(z^{\prime}\right) & \sim-\frac{1}{\left(z-z^{\prime}\right)^{2}}, \\
J_{+}(z) J_{-}\left(z^{\prime}\right) & \sim \frac{2 J_{3}}{z-z^{\prime}}-\frac{2}{\left(z-z^{\prime}\right)^{2}} .
\end{aligned}
$$

From the above OPE algebra, we learn that the $J$ 's furnish a module of an affine algebra of $S L(2)$ at level -2, which here, as noted in [11], appears in the Wakimoto free-field representation. Indeed, these chiral vertex operators are holomorphic in $z$, which means that one can expand them in a Laurent series that allows an affinisation of the $S L(2)$ algebra generated by their resulting zero modes. Thus, the space of global sections of $\widehat{\mathcal{O}}_{\mathbb{P}^{1}}^{c h}$ furnishes a module of an affine algebra of $S L(2)$ at level -2.2 case The space of these operators obeys all the physical axioms of a chiral algebra except for reparameterisation invariance on the $z$-plane or worldsheet $\Sigma$. We will substantiate this last statement momentarily by showing that the holomorphic stress tensor fails to exist in the $\bar{Q}_{+}$-cohomology at the quantum level. As we shall see shortly, this observation will be crucial to our results in this section.

\section{The Segal-Sugawara Tensor and the Classical Holomorphic Chiral Algebra}

Recall from section 2.6 and our $\bar{Q}_{+}$-Cech cohomology dictionary, that there will be a $\psi^{\bar{i}}$-independent stress tensor operator $T(z)$ in the quantum $\bar{Q}_{+}$-cohomology of the underlying twisted sigma-model on $\mathbb{C P}^{1}$, if and only if the corresponding $\widehat{T}(z)$ operator of the free $\beta \gamma$ system belongs in $H^{0}\left(\mathbb{C P}^{1}, \widehat{\mathcal{O}}_{\mathbb{P}^{1}}^{c h}\right)$ - the space of global sections of $\widehat{\mathcal{O}}_{\mathbb{P}^{1}}^{c h}$. Let's look at this more closely.

Now, note that for $X=\mathbb{C P}^{1}$, we have

$$
\widehat{T}(z)=-: \beta \partial_{z} \gamma:(z)
$$

\footnotetext{
${ }^{2}$ Note that one can consistently introduce appropriate fluxes to deform the level away from -2 - recall from our discussion in Appendix A that the $E_{i j}=\partial_{i} B_{j}$ term in A.30 is related to the fluxes that correspond to the moduli of the chiral algebra, and since this term will determine the level $k$ of the affine $S L(2)$ algebra via the term $-k \partial_{z} \gamma$ of $\tilde{\beta}$, (which is set to $k=-2$ in the current undeformed case), turning on the relevant fluxes will deform $k$ away from -2 . Henceforth, whenever we consider $k \neq-2$, we really mean turning on fluxes in this manner.
} 
where the above operators are defined and regular in $U_{1}$. Similarly, we also have

$$
\widetilde{T}(z)=-: \tilde{\beta} \partial_{z} \tilde{\gamma}:(z) .
$$

where the above operators are defined and regular in $U_{2}$. By substituting the automorphism relations (2.5) -(2.6) into (2.16), a small computation shows that in $U_{1} \cap U_{2}$, we have

$$
\widetilde{\widehat{T}}(z)-\widehat{T}(z)=\partial_{z}\left(\frac{\partial_{z} \gamma}{\gamma}\right) .
$$

where an operator that is a global section of $\widehat{\mathcal{O}}_{\mathbb{P}^{1}}^{c h}$ must agree in $U_{1} \cap U_{2}$.

The only way to consistently modify $\widehat{T}$ and $\widetilde{T}$ so as to agree on $U_{1} \cap U_{2}$, is to shift them by a multiple of the term $\left(\partial_{z}^{2} \gamma\right) / \gamma$ and $\left(\partial_{z} \gamma\right)^{2} / \gamma^{2}$. However, any linear sum of these two terms has a pole at both $\gamma=0$ and $\tilde{\gamma}=0$. Thus, it cannot be used to redefine $\widehat{T}$ or $\widetilde{T}$ (which has to be regular in $U_{1}$ or $U_{2}$ respectively). Therefore, we conclude that $\widehat{T}(z)$ does not belong in $H^{0}\left(\mathbb{C P}^{1}, \widehat{\mathcal{O}}_{\mathbb{P}^{1}}^{c h}\right)$. This means that $T(z)$ does not exist in the $\bar{Q}_{+}$-cohomology of the underlying twisted sigma-model on $\mathbb{C P}^{1}$ at the quantum level.

This last statement is in perfect agreement with the physical picture presented in section 2.3 , which states that since $c_{1}\left(\mathbb{C P}^{1}\right) \neq 0$, there are now one-loop corrections to the action of $\bar{Q}_{+}$, such that the $T(z)$ is no longer annihilated by $\bar{Q}_{+}$. This just corresponds to the mathematical fact that the sheaf $\widehat{\mathcal{O}}_{X}^{c h}$ of $\mathrm{CDO}$ on $X$ has a structure of a conformal vertex algebra if and only if the conformal anomaly measured by $c_{1}(X)$ vanishes. Note also that (2.17) is a counterpart in Cech cohomology to the sigma-model relation

$$
\left[\bar{Q}_{+}, T_{z z}\right]=\partial_{z}\left(R_{i \bar{j}} \partial_{z} \phi^{i} \psi^{\bar{j}}\right)
$$

Since $\phi^{i}$ corresponds to $\gamma^{i}$, we see from (2.18) that the sigma-model operator $R_{i \bar{j}} \psi^{\bar{j}}$ must correspond to $1 / \gamma$. Hence, we have an interpretation of the one-loop beta function (which is proportional to $R_{i \bar{j}}$ ) in terms of holomorphic data. This has been emphasised in [10] as a novel way to view the one-loop beta function from a purely mathematical viewpoint.

The absence of $T(z)$ in the quantum holomorphic chiral algebra of the twisted sigmamodel on $\mathbb{C P}^{1}$, can also be observed from a different but crucial viewpoint. To this end, note that for any affine algebra $\widehat{\mathfrak{g}}$ at level $k \neq-h^{\vee}$, where $h^{\vee}$ is the dual Coxeter number of the Lie algebra $\mathfrak{g}$, one can construct the corresponding stress tensor out of the currents of $\widehat{\mathfrak{g}}$ via a Segal-Sugawara construction [16]. In the present case of an affine $S L(2)$ algebra, the stress tensor can be constructed as

$$
T(z)=\frac{:\left(J_{+} J_{-}+J_{3}^{2}\right)(z):}{k+2}
$$


where because $\mathfrak{g}=\mathfrak{s l}_{2}, h^{\vee}=2$. As required, for every $k \neq-2$, the modes of the Laurent expansion of $T(z)$ will span a Virasoro algebra. In particular, $T(z)$ will generate holomorphic reparametrisations of the coordinates on the worldsheet $\Sigma$. Notice that this definition of $T(z)$ in (2.19) is ill-defined when $k=-2$. Nevertheless, one can always associate $T(z)$ with an operator $S(z)$ that is well-defined at any finite level, such that

$$
S(z)=(k+2) T(z)
$$

is known as the Segal-Sugawara tensor. It is given by

$$
S(z)=:\left(J_{+} J_{-}+J_{3}^{2}\right)(z):
$$

From (2.20), we see that $S(z)$ generates, in its OPE's with other field operators, $(k+2)$ times the transformations usually generated by the stress tensor $T(z)$. Therefore, at the level $k=-2, S(z)$ generates no transformations at all - its OPE's with all other field operators are trivial. This is equivalent to saying that the holomorphic stress tensor does not exist at all, since $S(z)$, which is the only well-defined operator at this level that could possibly generate the transformation of fields under an arbitrary holomorphic reparametrisation of the worldsheet coordinates on $\Sigma$, acts by zero.

Note that $T(z)$ will fail to exist in the chiral algebra and therefore $S(z)$ will act by zero, only at the quantum level, i.e., $T(z)$ and $S(z)$ still exist as local fields of spin two in the $\bar{Q}_{+}{ }^{-}$ cohomology of the sigma-model at the classical level. To substantiate this statement, first recall from section 2.3 that $\left[\bar{Q}_{+}, T(z)\right]=0$ classically in the absence of quantum corrections to the action of $\bar{Q}_{+}$. Next, note that the integer 2 in the factor $(k+2)$ of the expression $S(z)$ in (2.20), is due to a shift by $h^{\vee}=2$ in the level $k$ because of quantum renormalisation effects [17], i.e., the classical expression of $S(z)$ for a general level $k$ can actually be written as

$$
S(z)=k T(z)
$$

and therefore, one will also have $\left[\bar{Q}_{+}, S(z)\right]=0$ in the classical theory. Moreover, since in our case, we actually have $S(z)=-2 T(z)$ in the classical theory, it will also be true that under quantum corrections of the action of $\bar{Q}_{+}$, we will have

$$
\left[\bar{Q}_{+}, S_{z z}\right]=-2 \partial_{z}\left(R_{i \bar{j}} \partial_{z} \phi^{i} \psi^{\bar{j}}\right)
$$


This corresponds, in the Cech cohomology picture, to the expression

$$
\widetilde{\widehat{S}}(z)-\widehat{S}(z)=-2 \partial_{z}\left(\frac{\partial_{z} \gamma}{\gamma}\right)
$$

which means that $\widehat{S}(z)$, the Cech cohomology counterpart to the $S(z)$ operator, fails to be in $H^{0}\left(\mathbb{C P}^{1}, \widehat{\mathcal{O}}_{\mathbb{P}^{1}}^{c h}\right)$. This is again consistent with the fact that $S(z)$ does not belong in the quantum chiral algebra of the sigma-model, but rather, $S(z)$ belongs in its classical chiral algebra. In other words, one can always represent $S(z)$ by a classical $c$-number. This point will be important when we discuss how one can define Hecke eigensheaves that will correspond to flat ${ }^{L} G$-bundles on a Riemann surface $\Sigma$ in our physical interpretation of the geometric Langlands correspondence for $G=S L(2)$.

The fact that $S(z)$ fails to correspond to any element in $H^{0}\left(\mathbb{C P}^{1}, \widehat{\mathcal{O}}_{\mathbb{P}^{1}}^{c h}\right)$ means that it will act trivially in any OPE with other field operators. This in turn implies that its Laurent modes will commute with the Laurent modes of any other existing operator; in particular, the Laurent modes of $S(z)$ will commute with the Laurent modes of the currents $J_{+}(z)$, $J_{-}(z)$ and $J_{3}(z)$ - in other words, the Laurent modes of $S(z)$ will generate the centre $\mathfrak{z}\left(\widehat{\mathfrak{s l}}_{2}\right)$ of the completed universal enveloping algebra of the affine $S L(2)$ algebra $\widehat{\mathfrak{s l}}_{2}$ at the critical level $k=-2$ (spanned by the Laurent modes of $J_{+}(z), J_{-}(z)$ and $J_{3}(z)$ in the quantum chiral algebra of the twisted sigma-model on $S L(2) / B) \cdot 3$ Last but not least, notice that $S(z)$ is also $\psi^{\bar{j}}$-independent and must therefore be purely bosonic in nature. In other words, $S(z)$ exist only in the classical holomorphic chiral algebra of the purely bosonic (or $\psi^{\bar{j}}$-independent) sector of the twisted sigma-model.

Note that since $S(z)$ is a classical field, $\mathfrak{z}\left(\widehat{\mathfrak{s l}}_{2}\right)$, which is generated by its Laurent modes, must also be classical in nature. This statement can be further substantiated as follows. Firstly, note that since $S(z)$ is holomorphic in $z$ and is of conformal weight two, one can expand it in terms of a Laurent expansion as

$$
S(z)=\sum_{n \in \mathbb{Z}} \hat{S}_{n} z^{-n-2} .
$$

Let us begin with the general case of $k \neq-h^{\vee}$ for any affine algebra $\widehat{\mathfrak{g}}$, whereby a quantum definition of $S(z)$ exists, so that the $\hat{S}_{n}$ modes of its Laurent expansion can be related to the

\footnotetext{
${ }^{3}$ Recall that $S(z)$ is constructed out of the currents of the affine $S L(2)$ algebra by using the invariant tensors of the corresponding Lie algebra usually employed to define higher-order Casimir invariants. Consequently, its Laurent modes will span not the centre of the affine algebra, but rather the centre of the completed universal enveloping algebra of the affine algebra.
} 
$J_{n}^{a}$ modes of the currents of $\widehat{\mathfrak{g}}$ through the quantum commutator relations

$$
\begin{aligned}
& {\left[\hat{S}_{n}, J_{m}^{a}\right]=-\left(k+h^{\vee}\right) m J_{n+m}^{a},} \\
& {\left[\hat{S}_{n}, \hat{S}_{m}\right]=\left(k+h^{\vee}\right)\left((n-m) \hat{S}_{n+m}+\frac{k}{12} \operatorname{dim} \mathfrak{g}\left(n^{3}-n\right) \delta_{n,-m}\right),}
\end{aligned}
$$

where $a=1,2, \ldots$, dimg. If we now let $k=-h^{\vee}$ and $\mathfrak{g}=\mathfrak{s l}_{2}$, we will have $\left[\hat{S}_{n}, J_{m}^{a}\right]=$ $\left[\hat{S}_{n}, \hat{S}_{m}\right]=0$. Hence, one can define simultaneous eigenstates of the $\hat{S}_{n}$ and $J_{n}^{a}$ mode operators. In particular, one would be able to properly define a general state $\Psi=\hat{S}_{-l} \hat{S}_{-q} \ldots \hat{S}_{-p}|0, \alpha\rangle$, where $|0, \alpha\rangle$ is a vacuum state associated to a representation of $\mathfrak{s l}_{2}$ labelled by $\alpha$, such that $J_{0}^{a}|0, \alpha\rangle=\alpha^{a}|0, \alpha\rangle$. However, note that any $\Psi$ will correspond to a null-state, i.e., $\Psi$ decouples from the real, physical Hilbert space of quantum states spanned by the representations of $\mathfrak{s l}_{2}$ [18]. This means that the $\hat{S}_{m}$ 's which generate $\mathfrak{z}\left(\widehat{\mathfrak{s l}}_{2}\right)$ cannot exist as quantum mode operators. Hence, $\mathfrak{z}\left(\widehat{\mathfrak{s l}}_{2}\right)$ must be a classical algebra.

\section{A Classical Virasoro Algebra}

Since we now understand that $S(z)$ must be a holomorphic classical field at $k=-2$, let us rewrite, for interpretive clarity, the Laurent expansion of $S(z)$ as

$$
S(z)=\sum_{n \in \mathbb{Z}} S_{n} z^{-n-2},
$$

so as to differentiate the classical modes of expansion $S_{n}$ from their quantum counterpart $\hat{S}_{n}$ in (2.25). Unlike the $\hat{S}_{n}$ 's which obey the quantum commutator relations in (2.27) for an arbitrary level $k \neq-2$, the $S_{n}$ 's, being the modes of a Laurent expansion of a classical field, will instead obey Poisson bracket relations that define a certain classical algebra at $k=-2$.

Based on our arguments thus far, we see that the quantum version of $S(z)$ as expressed in (2.25), must reduce to its classical counterpart as expressed in (2.28), when $k \rightarrow-2$. In other words, one can see that by taking $(k+2) \rightarrow 0$, we are going to the classical limit of this operator. This is analogous to taking the limit $\hbar \rightarrow 0$ in any quantum mechanical theory so as to obtain its classical counterpart. In fact, by identifying $\left(k+h^{\vee}\right)$ or in this case $(k+2)$ with $i \hbar$, and by noting that one must make the replacement from Possion brackets to commutators via $\left\{S_{n}, S_{m}\right\}_{P . B .} \rightarrow \frac{1}{i \hbar}\left[\hat{S}_{n}, \hat{S}_{m}\right]$ in quantising the $S_{n}$ 's into operators, we can ascertain the classical algebra generated by the $S_{n}$ 's from (2.27) as

$$
\left\{S_{n}, S_{m}\right\}_{P . B .}=(n-m) S_{n+m}-\frac{6}{12}\left(n^{3}-n\right) \delta_{n,-m} .
$$


Since we have the classical relation $S(z) \sim T(z)$, it means that we can interpret the $S_{n}$ modes as the Virasoro modes of the Laurent expansion of the classical stress tensor field $T(z)$. In other words, the $S_{n}$ 's span a classical Virasoro algebra with central charge -6 as given by (2.29). This is sometimes denoted as the Virasoro Poisson algebra $\operatorname{Sym}^{\prime}\left(v_{-6}{ }_{-6}\right)$ in the mathematical literature [6]. Hence, we have the identification $\mathfrak{z}\left(\widehat{\mathfrak{s l}}_{2}\right) \simeq \operatorname{Sym}^{\prime}\left(\operatorname{vir}_{-6}\right)$

\subsection{A Gauged WZW Model and the Geometric Langlands Correspondence for $G=S L(2)$}

Let us now seek a dual description of the above classical, holomorphic chiral algebra of the twisted sigma-model on $S L(2) / B$ spanned by $S(z)$. To this end, let us first generalise the action of the twisted sigma-model by making the replacement $g_{i \bar{j}} \rightarrow g_{i \bar{j}}+b_{i \bar{j}}$ in $V$ of $S_{\text {twist }}$ in (A.9), where $b_{i \bar{j}}$ is a $(1,1)$-form on the target space $X$ associated to a B-field. This just adds to $S_{\text {twist }}$ a cohomologically-trivial $\bar{Q}_{+}$-exact term $\left\{\bar{Q}_{+},-b_{i j} \psi_{\bar{z}}^{i} \partial_{z} \phi^{\bar{j}}\right\}$, and does nothing to change our above discussions about the classical chiral algebra of the sigma-model. This generalised action can be explicitly written as

$$
S_{\text {gen }}=\int_{\Sigma}\left|d^{2} z\right|\left(g_{i \bar{j}}+b_{i \bar{j}}\right)\left(\partial_{z} \phi^{\bar{j}} \partial_{\bar{z}} \phi^{i}\right)+g_{i \bar{j}} \psi_{\bar{z}}^{i} D_{z} \psi^{\bar{j}}+b_{i \bar{j}} \psi_{\bar{z}}^{i} \partial_{z} \psi^{\bar{j}}+b_{i \bar{l}, \bar{j}} \psi_{\bar{z}}^{i} \partial_{z} \phi^{\bar{l}} \psi^{\bar{j}}
$$

Now recall that $S(z)$ exists in the classical holomorphic chiral algebra of the $\psi^{\bar{j}_{-}}$ independent, purely bosonic sector of the twisted sigma-model on $S L(2) / B$. This means that in order for one to ascertain the dual description of $S(z)$, it suffices to confine oneself to the study of the holomorphic chiral algebra of the $\psi^{\bar{j}}$-independent sector of the twisted sigma-model on $S L(2) / B$. The purely bosonic, $\psi^{\bar{j}}$-independent specialisation of $S_{\text {equiv }}$, which describes this particular sector of interest, can be written as

$$
S_{\text {bosonic }}=\int_{\Sigma}\left|d^{2} z\right|\left(g_{i \bar{j}}+b_{i \bar{j}}\right) \partial_{\bar{z}} \phi^{i} \partial_{z} \phi^{\bar{j}}
$$

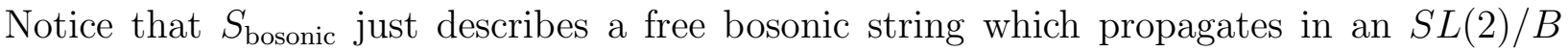
target-space. Hence, one can actually describe the holomorphic chiral algebra associated to the $\psi^{\bar{j}}$-independent sector of the twisted sigma-model on $S L(2) / B$ in terms of the holomorphic BRST-cohomology (or chiral algebra) of a $B$-gauged WZW model on $S L(2) 4$ In other words,

\footnotetext{
${ }^{4}$ Note that a non-linear sigma-model on any homogenous coset space such as $G / H$, will be described by an asymmetrically $H$-gauged WZW model on $G$ associated with the action $g \rightarrow g h^{-1}$, where $g \in G$ and $h \in H$. However, note that the BRST-cohomology of an asymmetrically $H$-gauged WZW model on $G$ coincides exactly with the holomorphic (i.e., purely left-moving) sector of the BRST-cohomology of a symmetrically $H$-gauged WZW model on $G$ that is genuinely gauge-invariant, and that which we are thus considering in
} 
$S(z)$ should correspond to an observable in the classical holomorphic BRST-cohomology of the $B$-gauged WZW model on $S L(2)$.

Note that what would be relevant to all our later discussions is the classical, holomorphic chiral algebra of the $\psi^{\bar{j}}$-independent, non-supersymmetric sector of the twisted sigma-model on $X=S L(N) / B$, for any $N \geq 3$. Note also that the above arguments would apply for all $X=S L(N) / B$. As such, let us now proceed to describe the $B$-gauged WZW model on any $S L(N)$ in greater detail 5

\section{The B-Gauged WZW Model on $S L(N)$}

First, note that the action of a general WZW model can be written as

$$
S_{\mathrm{WZ}}(g)=\frac{k^{\prime}}{4 \pi} \int_{\Sigma} d^{2} z \operatorname{Tr}\left(\partial_{z} g^{-1} \partial_{\bar{z}} g\right)+\frac{i k^{\prime}}{24 \pi} \int_{B ; \partial B=\Sigma} d^{3} x \operatorname{Tr}\left(g^{-1} d g\right)^{3}
$$

where $k^{\prime}$ is the level, and $g$ is a worldsheet scalar field valued in any simple, maximally non-compact, connected Lie group $G$ (such as $S L(N, \mathbf{C})$ which we are considering in this paper), that is also periodic along one of the worldsheet directions with period $2 \pi$. The trace $\mathrm{Tr}$ is the usual matrix trace in the defining representation of $G$.

A gauged version of (2.32) can be written as

$$
\begin{gathered}
S_{\text {gauged }}\left(g, A_{z}, A_{\bar{z}}\right)=S_{\mathrm{WZ}}(g)+\frac{k^{\prime}}{2 \pi} \int_{\Sigma} d^{2} z \operatorname{Tr}\left[A_{z}\left(\partial_{\bar{z}} g g^{-1}+\bar{M}\right)-A_{\bar{z}}\left(g^{-1} \partial_{z} g+M\right)\right. \\
\left.+A_{z} g A_{\bar{z}} g^{-1}-A_{z} A_{\bar{z}}\right],
\end{gathered}
$$

this paper. In other words, at the level of the holomorphic chiral algebra, a physically equivalent description of the $\psi^{\bar{j}}$-independent, non-supersymmetric sector of the twisted sigma-model on $S L(2) / B$, will be given by a $B$-gauged WZW model on $S L(2)$ that is gauge-invariant on the worldsheet. This argument applies for any $G=S L(N)$ and $H=B$ as well.

${ }^{5}$ It may be disconcerting to some readers that the Borel subgroup of $S L(N, \mathbf{C})$ which we are gauging the $S L(N, \mathbf{C})$ WZW model by, is non-compact in general. Apart from citing several well-known examples in the physical literature [13, 14, 19, 20, 21, that have done likewise to consider non-compact WZW models gauged to non-compact (sometimes Borel) subgroups, one can also argue that our model is actually equivalent within our context - to a physically consistent model which gauges a compact subgroup instead. Firstly, note that for a complex flag manifold $S L(N, \mathbf{C})$, we have the relation $S L(N, \mathbf{C}) / B=S U(N) / C(T)$, where $C(T)$ is the centralizer of the torus of $S U(N)$ spanned by purely diagonal matrices in $S U(N)$ [22] - in other words, $C(T)$ is an anomaly-free, compact diagonal subgroup in the context of a $C(T)$-gauged WZW model on $S U(N)$. Secondly, note that the OPE algebras of the affine algebras $\widehat{\mathfrak{s u}}_{N}$ and $\widehat{\mathfrak{s l}}_{N}$ are the same. Together with the previous footnote, these two points imply that the $B$-gauged WZW model on $S L(N, \mathbf{C})$ and the $C(T)$-gauged WZW model on $S U(N)$ (which can be physically consistently defined, and whose gauge group is also compact), are equivalent at the level of their holomorphic BRST-cohomologies. However, since one of our main aims is to relate the gauged WZW model to the algebraic DS-reduction scheme in $\S 3$, we want to consider the B-gauged WZW model on $S L(N, \mathbf{C})$. Last but not least, note that we will ultimately be interested in the classical spectrum of the gauged WZW model only, whereby the compactness or noncompactness of the gauge group will be irrelevant. 
where the worldsheet one-form gauge field $A=A_{z} d z+A_{\bar{z}} d \bar{z}$ is valued in $\mathfrak{h}$, the Lie algebra of a subgroup $H$ of $G$. Notice that $S_{\text {gauged }}\left(g, A_{z}, A_{\bar{z}}\right)$ differs slightly from the standard form of a gauged WZW model commonly found in the physical literature - additional $\bar{M}$ and $M$ constant matrices have been incorporated in the $\partial_{\bar{z}} g g^{-1}$ and $g^{-1} \partial_{z} g$ terms of the standard action, so that one can later use them to derive the correct form of the holomorphic stress tensor without reference to a coset formalism. Setting $\bar{M}$ and $M$ to the zero matrices simply takes us back to the standard action for the gauged WZW model. As required, $S_{\text {gauged }}\left(g, A_{z}, A_{\bar{z}}\right)$ is invariant under the standard (chiral) local gauge transformations

$$
g \rightarrow h g h^{-1} ; \quad A_{z} \rightarrow \partial_{z} h \cdot h^{-1}+h A_{z} h^{-1} ; \quad A_{\bar{z}} \rightarrow \partial_{\bar{z}} h \cdot h^{-1}+h A_{\bar{z}} h^{-1},
$$

where $h=e^{\lambda(z, \bar{z})} \in H$ for any $\lambda(z, \bar{z}) \in \mathfrak{h} 6$ The invariance of (2.33) under the gauge transformations in (2.34) can be verified as follows. Firstly, note that the $\bar{M}(M)$-independent terms make up the usual Lagrangian for the standard gauged WZW action, which is certainly invariant under the gauge transformations of (2.34). Next, note that under an infinitesimal gauge transformation $h \simeq 1+\lambda$, the terms $\operatorname{Tr}\left(A_{z} \bar{M}\right)$ and $\operatorname{Tr}\left(A_{\bar{z}} M\right)$ change as

$$
\begin{aligned}
& \delta \operatorname{Tr}\left(A_{z} \bar{M}\right)=\operatorname{Tr}\left(\partial_{z} \lambda \bar{M}\right)-\operatorname{Tr}\left(\bar{M}\left[\lambda, A_{z}\right]\right), \\
& \delta \operatorname{Tr}\left(A_{\bar{z}} M\right)=\operatorname{Tr}\left(\partial_{\bar{z}} \lambda M\right)-\operatorname{Tr}\left(M\left[\lambda, A_{\bar{z}}\right]\right) .
\end{aligned}
$$

Since we will be considering the case where $H$ is the Borel subgroup of $G$ and therefore, $\lambda$ and $A$ will be valued in the Lie algebra of a maximally solvable (Borel) subgroup of $G$, the second term on the R.H.S. of (2.35) and (2.36) will be zero [21]. What remains are total divergence terms that will vanish upon integration on $\Sigma$ because it is a worldsheet with no boundaries. Therefore, unless $H$ is a Borel subgroup of $G$ (or any other solvable subgroup of $G$ ), one cannot incorporate $\bar{M}$ and $M$ in the action and still maintain the requisite gauge invariance. This explains why generalisations of gauged WZW models with these constant matrices $\bar{M}$ and $M$ have not appeared much in the physical literature. Nevertheless, this generalisation can be considered in our case. As we shall see shortly, this generalisation will allow us to obtain the correct form of the holomorphic stress tensor of the $B$-gauged WZW model on $S L(N)$ without any explicit reference to a coset formalism.

\footnotetext{
${ }^{6} \mathrm{~A}$ similar model has been considered in [21. However, the action in that context is instead invariant under a non-chiral local gauge transformation. Moreover, it does not contain the $A_{z} A_{\bar{z}}$ term present in a standard gauged WZW model.
} 
The classical equations of motion that follow from the field variations in (2.34) are

$$
\begin{aligned}
\delta A_{z} & :\left.D_{\bar{z}} g g^{-1}\right|_{H}=-M_{+}, \\
\delta A_{\bar{z}} & :\left.g^{-1} D_{z} g\right|_{H}=-M- \\
\delta g & : D_{\bar{z}}\left(g^{-1} D_{z} g\right)=F_{z \bar{z}} \\
\delta g & : D_{z}\left(D_{\bar{z}} g g^{-1}\right)=F_{\bar{z} z},
\end{aligned}
$$

where $F_{z \bar{z}}=\partial_{z} A_{\bar{z}}-\partial_{\bar{z}} A_{z}+\left[A_{z}, A_{\bar{z}}\right]$ and $F_{\bar{z} z}=\partial_{\bar{z}} A_{z}-\partial_{z} A_{\bar{z}}+\left[A_{\bar{z}}, A_{z}\right]$ are the non-vanishing components of the field strength, and the covariant derivatives are given by $D_{z}=\partial_{z}+\left[A_{z},\right]$ and $D_{\bar{z}}=\partial_{\bar{z}}+\left[A_{\bar{z}},\right]$. By imposing the condition of (2.38) in (2.39), and by imposing the condition of (2.37) in (2.40), since $M_{ \pm}$are constant matrices, we find that we have the zero curvature condition $F_{z \bar{z}}=F_{\bar{z} z}=0$ as expected of a non-dynamically gauged WZW model. This means that $A_{z}$ and $A_{\bar{z}}$ are trivial on-shell. One is then free to use the gauge invariance to set $A_{z}$ and/or $A_{\bar{z}}$ to a constant such as zero. In setting $A_{z}=A_{\bar{z}}=0$ in (2.39) and (2.40), noting that $F_{z \bar{z}}=F_{\bar{z} z}=0$, we have the relations

$$
\partial_{\bar{z}}\left(g^{-1} \partial_{z} g\right)=0 \quad \text { and } \quad \partial_{z}\left(\partial_{\bar{z}} g g^{-1}\right)=0 .
$$

In other words, we have a $\mathfrak{g}$-valued, holomorphic conserved current $J(z)=g^{-1} \partial_{z} g$, and a $\mathfrak{g}$-valued antiholomorphic conserved current $\bar{J}(\bar{z})=\partial_{\bar{z}} g g^{-1}$, both of which are dimension one and generate affine symmetries on $\Sigma$. The action in (2.33) can thus be written as

$$
\begin{gathered}
S_{\text {gauged }}\left(g, A_{z}, A_{\bar{z}}\right)=S_{\mathrm{WZ}}(g)+\frac{k^{\prime}}{2 \pi} \int_{\Sigma} d^{2} z \operatorname{Tr}\left[A_{z}(\bar{J}(\bar{z})+\bar{M})-A_{\bar{z}}(J(z)+M)\right. \\
\left.+A_{z} g A_{\bar{z}} g^{-1}-A_{z} A_{\bar{z}}\right]
\end{gathered}
$$

For our case where $H$ is a Borel subgroup $B$ of $G$, one can further simplify (2.42) as follows. Firstly, since $G$ is a connected simple group, it will have a simple Lie algebra $\mathfrak{g}$. As such, $\mathfrak{g}$ will have a Cartan decomposition $\mathfrak{g}=\mathfrak{n}_{-} \oplus \mathfrak{c} \oplus \mathfrak{n}_{+}$, where $\mathfrak{c}$ is the Cartan subalgebra, and $\mathfrak{n}_{ \pm}$are the nilpotent subalgebras of the the upper and lower triangular matrices of $G$. The Borel subalgebras will then be given by $\mathfrak{b}_{ \pm}=\mathfrak{c} \oplus \mathfrak{n}_{ \pm}$, and they correspond to the Borel subgroups $B_{ \pm}$. For the complex flag manifolds that we will be considering in this paper, $B_{+}$will be the Borel subgroup of interest. $B$ will henceforth mean $B_{+}$in all of our proceeding discussions. With respect to this decomposition of the Lie algebra of $G$, we can write $J(z)=\sum_{a=1}^{\operatorname{dimn}_{-}} J_{-}^{a}(z) t_{a}^{-}+\sum_{a=1}^{\operatorname{dimc}} J_{c}^{a}(z) t_{a}^{c}+\sum_{a=1}^{\operatorname{dimn}_{+}} J_{+}^{a}(z) t_{a}^{+}$, and $\bar{J}(\bar{z})=$ $\sum_{a=1}^{\operatorname{dimn}_{-}} \bar{J}_{-}^{a}(\bar{z}) t_{a}^{-}+\sum_{a=1}^{\operatorname{dimc}} \bar{J}_{c}^{a}(\bar{z}) t_{a}^{c}+\sum_{a=1}^{\operatorname{dimn}_{+}} \bar{J}_{+}^{a}(\bar{z}) t_{a}^{+}$, where $t_{a}^{-} \in \mathfrak{n}_{-}, t_{a}^{c} \in \mathfrak{c}$, and $t_{a}^{+} \in \mathfrak{n}_{+}$. One 
can also write $M=\sum_{a=1}^{\operatorname{dimn}-} M_{-}^{a} t_{a}^{-}+\sum_{a=1}^{\operatorname{dimc}} M_{c}^{a} t_{a}^{c}+\sum_{a=1}^{\operatorname{dimn} n_{+}} M_{+}^{a} t_{a}^{+}$, and $\bar{M}=\sum_{a=1}^{\operatorname{dimn}-} \bar{M}_{-}^{a} t_{a}^{-}+$ $\sum_{a=1}^{\operatorname{dimc}} \bar{M}_{c}^{a} t_{a}^{c}+\sum_{a=1}^{\operatorname{dimn}{ }_{+}} \bar{M}_{+}^{a} t_{a}^{+}$, where $M_{ \pm ; c}^{a}\left(\bar{M}_{ \pm ; c}^{a}\right)$ are arbitrary number constants. Next, note that $H=B$, and $B \simeq N_{+}$, where $N_{+}=[B, B]$ is the subgroup of $G$ generated by its Lie algebra $\mathfrak{n}_{+}$of strictly upper triangular matrices which are traceless, i.e., for $t, t^{\prime} \in \mathfrak{n}_{+}$, we have $\operatorname{Tr}_{L}\left(t t^{\prime}\right)-\operatorname{Tr}_{R}\left(t^{\prime} t\right)=0$, where the trace $\operatorname{Tr}_{L}$ and $\operatorname{Tr}_{R}$ are taken over some $L$ and $R$ representation of $G$ respectively. In other words, $N_{+}$is the non-anomalous subgroup to be gauged, and we can write $A_{z}=\sum_{a=1}^{\operatorname{dimn}_{+}} \tilde{A}_{z}^{a} t_{a}^{+}$, and $A_{\bar{z}}=\sum_{a=1}^{\operatorname{dimn} n_{+}} \tilde{A}_{\bar{z}}^{a} t_{a}^{+}$. Next, note that since $\operatorname{Tr}\left(t_{a}^{\alpha} t_{b}^{\beta}\right)=\delta_{a, b} \delta^{\alpha, \beta}$, the trace of the second term on the R.H.S. of (2.42) will be non-vanishing only for components of $J(z)(\bar{J}(\bar{z}))$ and $M(\bar{M})$ that are associated to their expansion in $\mathfrak{n}_{+}$. Let us denote $J^{+}(z)=\sum_{a=1}^{\operatorname{dimn}_{+}} J_{+}^{a}(z) t_{a}^{+}$and $M^{+}=\sum_{a=1}^{\operatorname{dimn} n_{+}} M_{+}^{a} t_{a}^{+}$. Let us also denote $\bar{J}^{+}(\bar{z})=\sum_{a=1}^{\operatorname{dimn}_{+}} \bar{J}_{+}^{a}(\bar{z}) t_{a}^{+}$and $\bar{M}^{+}=\sum_{a=1}^{\operatorname{dimn} n_{+}} \bar{M}_{+}^{a} t_{a}^{+}$. Then, one can write the action of a $B$-gauged WZW model on $G=S L(N)$ as

$$
\begin{gathered}
S_{\mathrm{B} \text {-gauged }}\left(g, A_{z}, A_{\bar{z}}, J^{+}, \bar{J}^{+}\right)=S_{\mathrm{WZ}}(g)-\frac{k^{\prime}}{2 \pi} \int_{\Sigma} d^{2} z \operatorname{Tr}\left[A_{\bar{z}}\left(J^{+}(z)+M^{+}\right)-A_{z}\left(\bar{J}^{+}(\bar{z})+\bar{M}^{+}\right)\right. \\
\left.-A_{z} g A_{\bar{z}} g^{-1}+A_{z} A_{\bar{z}}\right] .
\end{gathered}
$$

The B-Gauged WZW Model on SL(2)

Now that we have derived the action $S_{\text {B-gauged }}\left(g, A_{z}, A_{\bar{z}}, J^{+}, \bar{J}^{+}\right)$of a $B$-gauged WZW model on any $S L(N)$, we will proceed to specialise to the case where $G=S L(2)$. In this case, $\operatorname{dim} \mathfrak{n}_{-}=\operatorname{dim} \mathfrak{c}=\operatorname{dim} \mathfrak{n}_{+}=1$, and so $J^{+}(z)=J_{+}^{1}(z) t_{1}^{+}, \bar{J}^{+}(z)=\bar{J}_{+}^{1}(z) t_{1}^{+}, M^{+}=M_{+}^{1} t_{1}^{+}$, $\bar{M}^{+}=\bar{M}_{+}^{1} t_{1}^{+}, A_{z}=\tilde{A}_{z}^{1}(z) t_{1}^{+}$and $A_{\bar{z}}=\tilde{A}_{\bar{z}}^{1} t_{1}^{+}$. The gauged WZW action is then given by

$$
\begin{gathered}
S_{S L(2)}\left(g, A_{z}, A_{\bar{z}}, J^{+}, \bar{J}^{+}\right)=S_{\mathrm{WZ}}(g)-\frac{k^{\prime}}{2 \pi} \int_{\Sigma} d^{2} z \tilde{A}_{\bar{z}}^{1}\left(J_{+}^{1}(z)+M_{+}^{1}\right)-\tilde{A}_{z}^{1}\left(\bar{J}_{+}^{1}(z)+\bar{M}_{+}^{1}\right) \\
-\tilde{A}_{z}^{1} g \tilde{A}_{\bar{z}}^{1} g^{-1}+\tilde{A}_{z}^{1} \tilde{A}_{\bar{z}}^{1} .
\end{gathered}
$$

Due to the $B$-gauge invariance of the theory, we must divide the measure in any path integral computation by the volume of the $B$-gauge symmetry. That is, the partition function has to take the form

$$
Z_{S L(2)}=\int_{\Sigma} \frac{\left[g^{-1} d g, d \tilde{A}_{z}^{1}, d \tilde{A}_{\bar{z}}^{1}\right]}{(\text { gauge volume })} \exp \left(i S_{S L(2)}\left(g, A_{z}, A_{\bar{z}}, J^{+}, \bar{J}^{+}\right)\right)
$$

One must now fix this gauge invariance to eliminate the non-unique degrees of freedom. One can do this by employing the BRST formalism which requires the introduction of FaddevPopov ghost fields. 
In order to obtain the holomorphic BRST transformations of the fields, one simply replaces the position-dependent infinitesimal gauge parameter $\epsilon$ of $h=B=\exp \left(-\epsilon t_{1}^{+}\right)$in the corresponding left-sector of the gauge transformations in (2.34) with the ghost field $c$, which then gives us

$$
\delta_{\mathrm{BRST}}(g)=-c t_{1}^{+} g, \quad \delta_{\mathrm{BRST}}\left(\tilde{A}_{\bar{z}}^{1}\right)=-\partial_{\bar{z}} c, \quad \delta_{\mathrm{BRST}}(\text { others })=0
$$

The ghost field $c$ and its anti-ghost partner $b$ will transform as

$$
\delta_{\mathrm{BRST}}(c)=0, \quad \delta_{\mathrm{BRST}}(b)=\tilde{B}, \quad \delta_{\mathrm{BRST}}(\tilde{B})=0
$$

In the above, $\tilde{B}$ is the Nakanishi-Lautrup auxiliary field that is the holomorphic BRST transform of $b$. It also serves as a Lagrange multiplier to impose the gauge-fixing condition.

In order to obtain the antiholomorphic BRST transformations of the fields, one employs the same recipe to the corresponding right-sector of the gauge transformations in (2.34) with the infinitesimal position-dependent gauge parameter now replaced by the ghost field $\bar{c}$, which then gives us

$$
\bar{\delta}_{\mathrm{BRST}}(g)=\bar{c} t_{1}^{+} g, \quad \bar{\delta}_{\mathrm{BRST}}\left(\tilde{A}_{z}^{1}\right)=-\partial_{z} \bar{c}, \quad \bar{\delta}_{\mathrm{BRST}}(\text { others })=0 .
$$

The ghost field $\bar{c}$ and its anti-ghost partner $\bar{b}$ will transform as

$$
\bar{\delta}_{\mathrm{BRST}}(\bar{c})=0, \quad \bar{\delta}_{\mathrm{BRST}}(\bar{b})=\tilde{\bar{B}}, \quad \bar{\delta}_{\mathrm{BRST}}(\tilde{\bar{B}})=0
$$

In the above, $\tilde{\bar{B}}$ is the Nakanishi-Lautrup auxiliary field that is the antiholomorphic BRST transform of $\bar{b}$. It also serves as a Lagrange multiplier to impose the gauge-fixing condition.

Since the BRST transformations in (2.46) and (2.48) are just infinitesimal versions of the gauge transformations in (2.34),$S_{S L(2)}\left(g, A_{z}, A_{\bar{z}}, J^{+}, \bar{J}^{+}\right)$will be invariant under them. An important point to note at this juncture is that in addition to $\left(\delta_{\mathrm{BRST}}+\bar{\delta}_{\mathrm{BRST}}\right) \cdot\left(\delta_{\mathrm{BRST}}+\right.$ $\left.\bar{\delta}_{\mathrm{BRST}}\right)=0$, the holomorphic and antiholomorphic BRST-variations are also separately nilpotent, i.e., $\delta_{\mathrm{BRST}}^{2}=0$ and $\bar{\delta}_{\mathrm{BRST}}^{2}=0$. Moreover, $\delta_{\mathrm{BRST}} \cdot \bar{\delta}_{\mathrm{BRST}}=-\bar{\delta}_{\mathrm{BRST}} \cdot \delta_{\mathrm{BRST}}$. In fact, one can easily inspect this from the field variations themselves. This means that the BRSTcohomology of the $B$-gauged WZW model on $S L(2)$ can be decomposed into independent holomorphic and antiholomorphic sectors that are just complex conjugate of each other, and that it can be computed via a spectral sequence, whereby the first two complexes will be 
furnished by its holomorphic and antiholomorphic BRST-cohomologies respectively. Since we will only be interested in the holomorphic chiral algebra of the $B$-gauged WZW model on $S L(2)$ (which as mentioned, is just identical to its antiholomorphic chiral algebra by a complex conjugation), we shall henceforth focus on the holomorphic BRST-cohomology of the $B$-gauged WZW model on $S L(2)$ (as well as for all other cases of $S L(N)$ in this paper, since this observation of a polarisation of the BRST-cohomology will be true of any $B$-gauged WZW model on $S L(N)$ as we will see.)

By the usual recipe of the BRST formalism, one can fix the gauge by adding to the BRST-invariant action $S_{S L(2)}\left(g, A_{z}, A_{\bar{z}}, J^{+}, \bar{J}^{+}\right)$, a BRST-exact term. Since the BRST transformation by $\left(\delta_{\mathrm{BRST}}+\bar{\delta}_{\mathrm{BRST}}\right)$ is nilpotent, the new total action will still be BRST-invariant as required. The choice of the BRST-exact operator will then define the gauge-fixing condition. A consistent choice of the BRST-exact operator will give us the requisite action for the ghost and anti-ghost fields - note that with

$$
S_{S L(2)}\left(g, A_{z}, A_{\bar{z}}, J^{+}, \bar{J}^{+}\right)+\left(\delta_{\mathrm{BRST}}+\bar{\delta}_{\mathrm{BRST}}\right)\left(\frac{k^{\prime}}{2 \pi} \int_{\Sigma} d^{2} z \tilde{A}_{\bar{z}}^{1} b+\tilde{A}_{z}^{1} \bar{b}\right)
$$

one will indeed have the desired total action, which can be written as

$$
\begin{array}{r}
S_{\mathrm{WZW}}(g)+\frac{k^{\prime}}{2 \pi} \int_{\Sigma} d^{2} z c \partial_{\bar{z}} b+\bar{c} \partial_{z} \bar{b}-\frac{k^{\prime}}{2 \pi} \int_{\Sigma} d^{2} z \tilde{A}_{\bar{z}}^{1}\left(J_{+}^{1}(z)+M_{+}^{1}-\tilde{B}\right)-\tilde{A}_{z}^{1}\left(\bar{J}_{+}^{1}(z)+\bar{M}_{+}^{1}+\tilde{\bar{B}}\right) \\
-\tilde{A}_{z}^{1} g \tilde{A}_{\bar{z}}^{1} g^{-1}+\tilde{A}_{z}^{1} \tilde{A}_{\bar{z}}^{1} .
\end{array}
$$

From the equation of motion by varying $\tilde{B}$, we have the condition $\tilde{A}_{\bar{z}}^{1}=0$. From the equation of motion by varying $\tilde{\bar{B}}$, we have the condition $\tilde{A}_{z}^{1}=0$. Together with the equation of motion by varying $\tilde{A}_{\bar{z}}^{1}$ and $\tilde{A}_{z}^{1}$, we have, by integrating out $\tilde{A}_{\bar{z}}^{1}$ and $\tilde{A}_{z}^{1}$ in (2.50), the relations $J_{+}^{1}+M_{+}^{1}=\tilde{B}$ and $\bar{J}_{+}^{1}+\bar{M}_{+}^{1}=-\tilde{\bar{B}}$. Thus, the partition function of the $B$-gauged WZW model can also be expressed as

$$
Z_{S L(2)}=\int\left[g^{-1} d g, d b, d c\right] \exp \left(i S_{\mathrm{WZW}}(g)+\frac{i k^{\prime}}{2 \pi} \int_{\Sigma} d^{2} z c \partial_{\bar{z}} b+\bar{c} \partial_{z} \bar{b}\right)
$$

whereby the holomorphic BRST variations of the fields that leave the effective action in $Z_{S L(2)}$ above invariant are now given by

$$
\delta_{\mathrm{BRST}}(g)=-c t_{1}^{+} g, \quad \delta_{\mathrm{BRST}}(c)=0, \quad \delta_{\mathrm{BRST}}(b)=\left(J_{+}^{1}+M_{+}^{1}\right), \quad \delta_{\mathrm{BRST}}(\text { others })=0 .
$$


The holomorphic BRST charge which generates the above transformations is therefore given by

$$
Q_{\mathrm{BRST}}=\oint \frac{d z}{2 \pi i}\left(J_{+}^{1}(z)+M_{+}^{1}\right) c(z)
$$

\section{The OPE's of the B-Gauged WZW Model on SL(2)}

Note that consistent with the presence of the dimension one operators $J_{ \pm}(z)$ and $J_{3}(z)$ in the holomorphic chiral algebra of the purely bosonic, $\psi^{\bar{j}}$-independent sector of the sigmamodel on $S L(2) / B$, which, generate an affine $S L(2)$ OPE algebra, one also has, in the holomorphic BRST-cohomology of the $B$-gauged WZW model on $S L(2)$, the dimension one currents $J_{ \pm}^{1}(z)$ and $J_{c}^{1}(z)$ which generate the following affine $S L(2)$ OPE algebra:

$$
\begin{aligned}
J_{c}^{1}(z) J_{ \pm}^{1}\left(z^{\prime}\right) & \sim \frac{ \pm J_{ \pm}^{1}\left(z^{\prime}\right)}{z-z^{\prime}} \\
J_{c}^{1}(z) J_{c}^{1}\left(z^{\prime}\right) & \sim \frac{k^{\prime} / 2}{\left(z-z^{\prime}\right)^{2}}, \\
J_{+}^{1}(z) J_{-}^{1}\left(z^{\prime}\right) & \sim \frac{2 J_{c}^{1}}{z-z^{\prime}}+\frac{k^{\prime}}{\left(z-z^{\prime}\right)^{2}} .
\end{aligned}
$$

From standard field-theoretic considerations of the ghost/anti-ghost kinetic term in the effective action of the gauged WZW model in (2.51), one will also have the following OPE (after absorbing $k^{\prime}$ by a trivially re-scaling of the fields)

$$
b(z) c\left(z^{\prime}\right) \sim \frac{1}{z-z^{\prime}} .
$$

However, the OPE's of the $b(z)$ and $c(z)$ fields with any current in (2.54)-(2.56) are trivial. Finally, one can also verify the nilpotency of $Q_{\mathrm{BRST}}$ by using the OPE's in (2.54)-(2.56), (2.57), and its explicit expression in (2.53) - the OPE of the BRST current $\left(J_{+}^{1}(z)+M_{+}^{1}\right) c(z)$ with itself is regular. Moreover, one can quickly check using (2.57) that $Q_{\mathrm{BRST}}$ in (2.53) will indeed generate the correct field variations in (2.52).

\section{The Holomorphic Stress Tensor}

Though we did not make this obvious in our discussion above, $\tilde{B}$ must actually vanish - by integrating out $\tilde{A}_{\bar{z}}^{1}$ in $(2.50)$ and using the condition $\tilde{A}_{z}^{1}=0$, we find that we actually have the relation $\left(J_{+}^{1}(z)+M_{+}^{1}\right)=0$. This relation (involving the current associated to the Borel subalgebra $\mathfrak{b}$ of the group $B$ that we are modding out by) will lead us directly to the correct form of the holomorphic stress tensor for the gauged WZW model without reference to a coset formalism. Let us look at this more closely. 
Since we have an affine $S L(2)$ algebra from the OPE's (2.54)-(2.56), we can employ the Sugawara formalism to construct the stress tensor associated with the $S_{\mathrm{WZW}}(g)$ part of the total action, from the currents in (2.54)-(2.56). Taking into account the part of the total action associated to the ghost and anti-ghost fields $c(z)$ and $b(z)$, the stress tensor should be given by

$$
T_{\text {gauged }}(z)=T_{S L(2)}(z)+\partial_{z} b(z) c(z)
$$

where

$$
T_{S L(2)}(z)=\frac{: d^{a b} J_{a}^{1} J_{b}^{1}(z):}{\left(k^{\prime}+2\right)},
$$

and $d^{a b}$ is the inverse of the Cartan-Killing metric of $\mathfrak{s l}_{2}$. Note that with respect to $T_{\text {gauged }}(z)$, the currents $J_{ \pm}^{1}(z)$ and $J_{c}^{1}(z)$ have conformal dimension one. This is inconsistent with the condition $J_{+}^{1}(z)=-M_{+}^{1}$, as $M_{+}^{1}$ is a constant of conformal dimension zero. This means that one must modify $T_{\text {gauge }}(z)$ so that $J_{+}^{1}(z)$ will have conformal dimension zero. An allowable modification involves adding to $T_{\text {gauge }}(z)$ a term that has conformal dimension two. A little thought will reveal that the total stress tensor must then take the form

$$
T_{\text {total }}(z)=T_{S L(2)}(z)+\partial_{z} b(z) c(z)+\partial_{z} J_{c}^{1}(z)
$$

A small computation shows that the BRST current $\left(J_{+}^{1}(z)+M_{+}^{1}\right) c(z)$ has conformal dimension one under $T_{\text {total }}(z)$, which then means that its $Q_{\mathrm{BRST}}$ charge is a conformal dimension zero scalar as required. This in turn means that $c(z)$ and $b(z)$ must be of conformal dimension one and zero respectively, i.e., the field $b(z)$ and $c(z)$ is a scalar and (holomorphic) one-form on $\Sigma$. Therefore, one should really rewrite $c(z)$ as $c_{z}(z)$ in (2.51), (2.53), (2.58) and (2.60). In doing so, we find that these equations are now fully consistent with regards to conformal dimensions.

Note also that because the BRST current is of conformal dimension one with respect to the holomorphic stress tensor $T_{\text {total }}(z)$, it must be annihilated by $Q_{\mathrm{BRST}}$; this means that $T_{\text {total }}(z)$ is $Q_{\mathrm{BRST}}$-closed. One can also verify that $T_{\text {total }}(z)$ cannot be $Q_{\mathrm{BRST}}$-exact, i.e., $T_{\text {total }}(z)$ lies in the holomorphic BRST-cohomology of the $B$-gauged WZW model on $S L(2)$. Last but not least, a soon-to-be relevant point to note is that since quantum corrections can only annihilate classes in the BRST-cohomology and not create them, the classical counterpart of the holomorphic stress tensor $T_{\text {total }}(z)$ will be a spin-two field $T_{\text {classical }}(z)$ which lies in the classical, holomorphic BRST-cohomology (or holomorphic chiral algebra) of the $B$-gauged WZW model on $S L(2)$, where $T_{\text {classical }}(z)$ will generate the classical Virasoro transformations on the fields. 
A Duality of Classical $\mathcal{W}$-algebras Underlying a Geometric Langlands Correspondence for $G=S L(2)$

Note that the observable in the holomorphic BRST-cohomology of the $B$-gauged WZW model on $S L(2)$ that will correspond to $S(z)$ of the holomorphic chiral algebra of the purely bosonic, $\psi^{\bar{j}}$-independent sector of the twisted sigma-model on $S L(2) / B$, must have the same spin as $S(z)$. In addition, since $S(z)$ generates a classical Virasoro symmetry on the worldsheet, it will mean that the corresponding observable ought to be a spin-two field which exists in the classical, holomorphic BRST-cohomology of the B-gauged WZW model on $S L(2)$, and which also generates a classical Virasoro symmetry on the worldsheet.

In order to ascertain the classical observable which corresponds to $S(z)$, first recall that the quantum definition of $S(z)$ at $k \neq-2$ is given by $S(z)=(k+2) T(z)$. Notice that since the stress tensor $T(z)$ also exists in the holomorphic chiral algebra of the purely bosonic, $\psi^{\bar{j}_{-}}$ independent sector of the sigma-model on $S L(2) / B$, it will imply that $T(z)$ must correspond to the stress tensor $T_{\text {total }}(z)$ of the $B$-gauged WZW model on $S L(2)$. Thus, at $k \neq-2$, $S(z)$ will correspond to $\bar{T}_{\text {total }}(z)=(k+2) T_{\text {total }}(z)$, and at $k=-2, S(z)$ will correspond to the classical counterpart $\bar{T}_{\text {classical }}(z)$ of $\bar{T}_{\text {total }}(z)$. Note that $\bar{T}_{\text {classical }}(z)$ lies in the classical, holomorphic BRST-cohomology of the $B$-gauged WZW model on $S L(2)$ as required - at $k=-2, \bar{T}_{\text {total }}(z)$, which usually exists as a quantum operator, will act by zero in its OPE's with any other operator, i.e., it will reduce to its classical counterpart $\bar{T}_{\text {classical }}(z)$ in the classical, holomorphic BRST-cohomology of the gauged WZW model. Moreover, since the shift in 2 in the factor $(k+2)$ is due to a quantum effect as explained earlier, $S(z)$ will actually correspond to $\bar{T}_{\text {classical }}(z)=-2 T_{\text {classical }}(z)$ at $k=-2$. Hence, $S(z)$ will indeed correspond to a spin-two field $\bar{T}_{\text {classical }}(z)$ in the classical, holomorphic BRST-cohomology of the $B$-gauged WZW on $S L(2)$ which generates a classical Virasoro transformation of the fields.

What is the classical algebra generated by the Laurent modes of $\bar{T}_{\text {classical }}(z)$ ? To ascertain this, first note that from the explicit form of $T_{\text {total }}(z)$ in (2.60), we find that it has to have a central charge of $c=13-6 / k^{\prime}+2-6\left(k^{\prime}+2\right)$. Hence, the Virasoro modes of $T_{\text {total }}(z)=\sum_{n} \hat{L}_{n} z^{-n-2}$ will obey the following commutator relation

$$
\left[\hat{L}_{n}, \hat{L}_{m}\right]=(n-m) \hat{L}_{n+m}+\frac{1}{12}\left[13-\frac{6}{k^{\prime}+2}-6\left(k^{\prime}+2\right)\right]\left(n^{3}-n\right) \delta_{n,-m}
$$

at the quantum level. Therefore, the commutator relations involving the $\hat{\bar{L}}_{n}$ modes of 
$\bar{T}_{\text {total }}(z)=\sum_{n} \hat{\bar{L}}_{n} z^{-n-2}$ will be given by

$\left[\hat{\bar{L}}_{n}, \hat{\bar{L}}_{m}\right]=(k+2)\left[(n-m) \hat{\bar{L}}_{n+m}+\left(n^{3}-n\right) \delta_{n,-m}\left(\frac{13(k+2)}{12}-\frac{6(k+2)}{12\left(k^{\prime}+2\right)}-\frac{6(k+2)\left(k^{\prime}+2\right)}{12}\right)\right]$.

At $k=-2, \bar{T}_{\text {total }}(z)$ will cease to have a quantum definition, and it will reduce to its classical counterpart $\bar{T}_{\text {classical }}(z)$. Consequently, the $k \rightarrow-2$ (and $k^{\prime} \rightarrow \infty$ ) limit of the commutator relation in (2.62), can be interpreted as its classical limit. Therefore, one can view the term $(k+2)$ in $(2.62)$ as the parameter $i \hbar$, where $\hbar \rightarrow 0$ is equivalent to the classical limit of the commutator relations. Since in a quantisation procedure, we go from $\left\{\bar{L}_{n}, \bar{L}_{m}\right\}_{P . B .} \rightarrow \frac{1}{i \hbar}\left[\hat{\bar{L}}_{n}, \hat{\bar{L}}_{m}\right]$, going in reverse would give us the classical Poisson bracket relation

$$
\left\{\bar{L}_{n}, \bar{L}_{m}\right\}_{P . B .}=(n-m) \bar{L}_{n+m}-\frac{6}{12}(k+2)\left(k^{\prime}+2\right)\left(n^{3}-n\right) \delta_{n,-m},
$$

where $\bar{T}_{\text {classical }}(z)=\sum_{n} \bar{L}_{n} z^{-n-2}$. Since the Poisson bracket must be well-defined as $k \rightarrow-2$ and $k^{\prime} \rightarrow \infty$, it will mean that $(k+2)\left(k^{\prime}+2\right)$ must be equal to a finite constant $l$ in these limits. For different values of $l$, the Poisson algebra generated by the Laurent modes of $\bar{T}_{\text {classical }}(z)$, will be a classical Virasoro algebra with different central charges. Note at this point that an equivalence - at the level of the holomorphic chiral algebra - between the $\psi^{\bar{j}_{-}}$ independent sector of the twisted sigma-model on $S L(2) / B$ and the $B$-gauged WZW model on $S L(2)$, will mean that the Laurent modes of $\bar{T}_{\text {classical }}(z)$ and $S(z)$ ought to generate an isomorphic classical algebra. In other words, the $\bar{L}_{m}$ 's ought to generate the same classical Virasoro algebra with central charge -6 that is generated by the Laurent modes $S_{m}$ of the sigma-model description in (2.29), i.e., we must have $l=1$, or rather $(k+2)=1 /\left(k^{\prime}+2\right)$. Thus, the Poisson algebra generated by the $\bar{L}_{m}$ 's must be given by

$$
\left\{\bar{L}_{n}, \bar{L}_{m}\right\}_{P . B .}=(n-m) \bar{L}_{n+m}-\frac{6}{12}\left(n^{3}-n\right) \delta_{n,-m} .
$$

This algebra also coincides with $\mathcal{W}_{\infty}\left(\widehat{\mathfrak{s l}}_{2}\right)$, the classical $\mathcal{W}$-algebra associated to $\widehat{\mathfrak{s l}}_{2}$ at level $k^{\prime} \rightarrow \infty$ obtained via a DS reduction scheme [7]. Since the $S_{m}$ 's that correspond to the $\bar{L}_{m}$ 's in (2.64) $\operatorname{span} \mathfrak{z}\left(\widehat{\mathfrak{s l}}_{2}\right)$, and since for $\mathfrak{g}=\mathfrak{s l}_{2}={ }^{L} \mathfrak{g}$, we have $h^{\vee}={ }^{L} h^{\vee}=2$, and $r^{\vee}=1$, where $r^{\vee}$ is the lacing number of $\mathfrak{g}$, we find that an equivalence - at the level of the holomorphic chiral algebra - between the $\psi^{\bar{j}}$-independent sector of the twisted sigma-model on $S L(2) / B$ and the $B$-gauged WZW model on $S L(2)$, will imply an isomorphism of Poisson algebras

$$
\mathfrak{z}(\widehat{\mathfrak{g}}) \cong \mathcal{W}_{\infty}\left({ }^{L} \widehat{\mathfrak{g}}\right),
$$


and the level relation

$$
\left(k+h^{\vee}\right) r^{\vee}=\frac{1}{\left(k^{\prime}+{ }^{L} h^{\vee}\right)} .
$$

Note at this point that the purely bosonic, $\psi^{\bar{j}}$-independent sector of the twisted sigma-model on $S L(2) / B$, can be described, via (2.31), by a bosonic string on $S L(2) / B$. On the other hand, note that since a bosonic string on a group manifold $G$ can be described as a WZW model on $G$, it will mean that the $B$-gauged WZW model on $S L(2)$ can be interpreted as a $B$-gauged bosonic string on $S L(2)$. Thus, we see that an equivalence, at the level of the holomorphic chiral algebra, between a bosonic string on $S L(2) / B$ and a $B$-gauged version of itself on $S L(2)$ - a statement which stems from the ubiquitous notion that one can always physically interpret a geometrical symmetry of the target space as a gauge symmetry in the worldsheet theory - will imply an isomorphism of classical $\mathcal{W}$-algebras and a level relation which underlie a geometric Langlands correspondence for $G=S L(2)$ ! Notice that the correspondence between the $k \rightarrow-2$ and $k^{\prime} \rightarrow \infty$ limits (within the context of the above Poisson algebras) is indeed consistent with the relation (2.66). These limits define a "classical" geometric Langlands correspondence. A "quantum" generalisation of the $S L(2)$ correspondence can be defined for other values of $k$ and $k^{\prime}$ that satisfy the relation (2.66) but with the isomorphism of (2.65) replaced by an isomorphism of quantum $\mathcal{W}$-algebras (derived from a DS-reduction scheme) associated to $\widehat{\mathfrak{s l}}_{2}$ at levels $k$ and $k^{\prime}$ respectively [6].

\subsection{The Twisted Sigma-Model on $S L(3) / B$ and its Classical Holomorphic Chiral Algebra}

Now, let us take $X=S L(3) / B$, where $B$ is the subgroup of upper triangular matrices of $S L(3)$ with a nilpotent Lie algebra $\mathfrak{b}$. Note that $\operatorname{dim}_{\mathbb{C}} X=3$, and one can cover $X$ with six open charts $U_{w}$ where $w=1,2, \ldots, 6$, such that each open chart $U_{w}$ can be identified with the affine space $\mathbb{C}^{3}$. Hence, the sheaf of CDO's in any $U_{w}$ can be described by three free $\beta \gamma$ systems with the action

$$
I=\sum_{i=1}^{3} \frac{1}{2 \pi} \int\left|d^{2} z\right| \beta_{i} \partial_{\bar{z}} \gamma^{i}
$$

As before, the $\beta_{i}$ 's and $\gamma^{i}$ 's are fields of dimension $(1,0)$ and $(0,0)$ respectively. They obey the standard free-field OPE's; there are no singularities in the operator products $\beta_{i}(z) \cdot \beta_{i}\left(z^{\prime}\right)$ and $\gamma^{i}(z) \cdot \gamma^{i}\left(z^{\prime}\right)$, while

$$
\beta_{i}(z) \gamma^{j}\left(z^{\prime}\right) \sim-\frac{\delta_{i}^{j}}{z-z^{\prime}}
$$


Similarly, the sheaf of CDO's in a neighbouring intersecting chart $U_{w+1}$ is described by three free $\tilde{\beta} \tilde{\gamma}$ systems with action

$$
I=\sum_{i=1}^{3} \frac{1}{2 \pi} \int\left|d^{2} z\right| \tilde{\beta}_{i} \partial_{\bar{z}} \tilde{\gamma}^{i}
$$

where the $\tilde{\beta}_{i}$ and $\tilde{\gamma}^{i}$ fields obey the same OPE's as the $\beta_{i}$ and $\gamma^{i}$ fields. In other words, the non-trivial OPE's are given by

$$
\tilde{\beta}_{i}(z) \tilde{\gamma}^{j}\left(z^{\prime}\right) \sim-\frac{\delta_{i}^{j}}{z-z^{\prime}}
$$

In order to describe a globally-defined sheaf of CDO's, one will need to glue the free conformal field theories with actions (2.67) and (2.69) in the overlap region $U_{w} \cap U_{w+1}$ for every $w=1,2, \ldots 6$, where $U_{7}=U_{1}$. To do so, one must use the admissible automorphisms of the free conformal field theories defined in (A.29)-(A.30) to glue the free-fields together. In the case of $X=S L(3) / B$, the relation between the coordinates in $U_{w}$ and $U_{w+1}$ will mean that the $\tilde{\gamma}^{i}$ 's in $U_{w+1}$ will be related to the $\gamma^{i}$ s in $U_{w}$ via the relation $[\tilde{\gamma}]=\left[V_{w+1}\right]^{-1}\left[V_{w}\right][\gamma]$, where the $3 \times 3$ matrices $\left[V_{w+1}\right]$ and $\left[V_{w}\right]$ are elements of the $S_{3}$ permutation subgroup of $G L(3)$ matrices associated to the open charts $U_{w+1}$ and $U_{w}$ respectively, and $[\gamma]$ is a $3 \times 1$ column matrix with the $\gamma^{i}$ 's as entries. By substituting this relation between the $\tilde{\gamma}^{i}$ 's and $\gamma^{i}$ s in (A.29)-(A.30), one will have the admissible automorphisms of the fields, which can then be used to glue together the local sheaves of CDO's in the overlap region $U_{w} \cap U_{w+1}$ for every $w=1,2, \ldots, 6$. These gluing relations for the free fields can be written as

$$
\begin{aligned}
& \tilde{\gamma}^{i}=\left[V_{w+1}^{-1} \cdot V_{w}\right]_{j}^{i} \gamma^{j}, \\
& \tilde{\beta}_{i}=\beta_{k} D_{i}^{k}+\partial_{z} \gamma^{j} E_{i j},
\end{aligned}
$$

where $i, j, k=1,2, \ldots, 3$. Here, $D$ and $E$ are $3 \times 3$ matrices, whereby $\left[\left(D^{T}\right)^{-1}\right]_{i}{ }^{k}=\partial_{i}\left[V_{w+1}^{-1}\right.$. $\left.V_{w}\right]^{k}{ }_{j} \gamma^{j}$ and $[E]_{i j}=\partial_{i} B_{j}$. It can be verified that $\tilde{\beta}$ and $\tilde{\gamma}$ obey the correct OPE's amongst themselves. Moreover, let $R_{w}$ represent a transformation of the fields in going from $U_{w}$ to $U_{w+1}$. One can indeed verify that just as in the previous case where we considered constructing a sheaf of CDO's on $S L(2) / B$, there is no anomaly to a global definition of a sheaf of CDO's on $X=S L(3) / B$ - a careful computation will reveal that one will get the desired composition maps $\left(R_{6} R_{5} R_{4} R_{3} R_{2} R_{1}\right) \cdot \gamma^{j}=\gamma^{j}$ and $\left(R_{6} R_{5} R_{4} R_{3} R_{2} R_{1}\right) \cdot \beta_{i}=\beta_{i}$. Again, this is just a statement that one can always define a sheaf of CDO's on any flag manifold $S L(N) / B[11$. 
Global Sections of the Sheaf of CDO's on $X=S L(3) / B$

Since $X=S L(3) / B$ is of complex dimension 3, the chiral algebra $\mathcal{A}$ will be given by $\mathcal{A}=\bigoplus_{g_{R}=0}^{g_{R}=3} H^{g_{R}}\left(X, \widehat{\mathcal{O}}_{X}^{c h}\right)$ as a vector space. As before and throughout this paper, it would suffice for our purpose to concentrate on just the purely bosonic sector of $\mathcal{A}$ - from our $\bar{Q}_{+}$-Cech cohomology dictionary, this again translates to studying only the global sections in $H^{0}\left(X, \widehat{\mathcal{O}}_{X}^{c h}\right)$.

According to theorem 5.13 of [11], one can always find elements in $H^{0}\left(M, \widehat{\mathcal{O}}_{M}^{c h}\right)$ for any flag manifold $M=S L(N) / B$, that will furnish a module of an affine $S L(N)$ algebra at the critical level. This means that one can always find dimension one global sections of the sheaf $\widehat{\mathcal{O}}_{X}^{c h}$ that correspond to $\psi^{\bar{i}}$-independent currents $J^{a}(z)$ for $a=1,2, \ldots \operatorname{dim} \mathfrak{s l}_{3}=8$, that satisfy the OPE's of an affine $S L(3)$ algebra at the critical level $k=-3$ :

$$
J_{a}(z) J_{b}\left(z^{\prime}\right) \sim-\frac{3 d_{a b}}{\left(z-z^{\prime}\right)^{2}}+\sum_{c} f_{a b}{ }^{c} \frac{J_{c}\left(z^{\prime}\right)}{\left(z-z^{\prime}\right)}
$$

where $d_{a b}$ is the Cartan-Killing metric of $\mathfrak{s l}_{3} 7$ Since these are global sections, it will be true that $\widetilde{J}_{a}(z)=J_{a}(z)$ on any $U_{w} \cap U_{w+1}$ and $a$. Moreover, from our $\bar{Q}_{+}$-Cech cohomology dictionary, they will be $\bar{Q}_{+}$-closed chiral vertex operators that are holomorphic in $z$, which means that one can expand them in a Laurent series that allows an affinisation of the $S L(3)$ algebra generated by their resulting zero modes. Similar to the $S L(2) / B \simeq \mathbb{C P}^{1}$ case, the space of these operators obeys all the physical axioms of a chiral algebra except for reparameterisation invariance on the $z$-plane or worldsheet $\Sigma$. We will substantiate this last statement next by showing that the holomorphic stress tensor fails to exist in the $\bar{Q}_{+}{ }^{-}$ cohomology at the quantum level. Again, this observation will be important in our discussion of a geometric Langlands correspondence for $G=S L(3)$.

\section{The Segal-Sugawara Tensor and the Classical Holomorphic Chiral Algebra}

Recall that for any affine algebra $\widehat{\mathfrak{g}}$ at level $k \neq-h^{\vee}$, where $h^{\vee}$ is the dual Coxeter number of the Lie algebra $\mathfrak{g}$, one can construct the corresponding stress tensor out of the currents of $\widehat{\mathfrak{g}}$ via a Segal-Sugawara construction [16]. In the present case of an affine $S L(3)$

\footnotetext{
${ }^{7}$ Note that one can consistently introduce appropriate fluxes to deform the level away from -3 - recall from our discussion in $\S$ A.7 that the $E_{i j}=\partial_{i} B_{j}$ term in (2.72) is related to the fluxes that correspond to the moduli of the chiral algebra, and since this term will determine the level $k$ of the affine $S L(3)$ algebra via the identification of the global sections $\tilde{\beta}_{i}$ with the affine currents valued in the subalgebra of $\mathfrak{s l}_{3}$ associated to its positive roots, turning on the relevant fluxes will deform $k$ away from -3 . Henceforth, whenever we consider $k \neq-3$, we really mean turning on fluxes in this manner.
} 
algebra, the stress tensor can be constructed as

$$
T(z)=\frac{: d^{a b} J_{a} J_{b}(z):}{k+3}
$$

where $d^{a b}$ is the inverse of the Cartan-Killing metric of $\mathfrak{s l}_{3}$, and $h^{\vee}=3$. As required, for every $k \neq-3$, the modes of the Laurent expansion of $T(z)$ will span a Virasoro algebra. In particular, $T(z)$ will generate holomorphic reparametrisations of the coordinates on the worldsheet $\Sigma$. Notice that this definition of $T(z)$ in (2.74) is ill-defined when $k=-3$. Nevertheless, one can always associate $T(z)$ with the Segal-Sugawara operator $S(z)$ that is well-defined at any finite level, whereby

$$
S(z)=(k+3) T(z)
$$

and

$$
S(z)=: d^{a b} J_{a} J_{b}(z):
$$

From (2.75), we see that $S(z)$ generates, in its OPE's with other field operators, $(k+3)$ times the transformations usually generated by the stress tensor $T(z)$. Therefore, at the level $k=-3, S(z)$ generates no transformations at all - its OPE's with all other field operators are trivial. This is equivalent to saying that the holomorphic stress tensor does not exist at the quantum level, since $S(z)$, which is the only well-defined operator at this level that could possibly generate the transformation of fields under an arbitrary holomorphic reparametrisation of the worldsheet coordinates on $\Sigma$, acts by zero in the OPE's.

Despite the fact that $S(z)$ will cease to exist in the spectrum of physical operators associated to the twisted sigma-model on $X=S L(3) / B$ at the quantum level, it will nevertheless exist as a field in its classical $\bar{Q}_{+}$-cohomology or holomorphic chiral algebra. One can convince oneself that this is true as follows. Firstly, from our $\bar{Q}_{+}$-Cech cohomology dictionary, since the $J_{a}(z)$ 's are in $H^{0}\left(X, \widehat{\mathcal{O}}_{X}^{c h}\right)$, it will mean that they are in the $\bar{Q}_{+}$-cohomology of the sigma-model at the quantum level. Secondly, since quantum corrections can only annihilate cohomology classes and not create them, it will mean that the $J_{a}(z)$ 's will be in the classical $\bar{Q}_{+}$-cohomology of the sigma-model, i.e., the currents are $\bar{Q}_{+}$-closed and are therefore invariant under the transformations generated by $\bar{Q}_{+}$in the absence of quantum corrections. Hence, one can readily see that (the classical counterpart of) $S(z)$ in (2.76) will also be $\bar{Q}_{+}$-closed at the classical level. Lastly, recall from section 2.3 that $\left[\bar{Q}_{+}, T(z)\right]=0$ such that $T(z) \neq\left\{\bar{Q}_{+}, \cdots\right\}$ in the absence of quantum corrections to the action of $\bar{Q}_{+}$in the classical 
theory. Note also that the integer 3 in the factor $(k+3)$ of the expression $S(z)$ in (2.75), is due to a shift by $h^{\vee}=3$ in the level $k$ because of quantum renormalisation effects [17], i.e., the classical expression of $S(z)$ for a general level $k$ can actually be written as $S(z)=k T(z)$, and therefore, one will have $\left[\bar{Q}_{+},-3 T(z)\right]=\left[\bar{Q}_{+}, S(z)\right]=0$, where $S(z) \neq\left\{\bar{Q}_{+}, \cdots\right\}$ in the classical theory. Therefore, $S(z)$ will be a spin-two field in the classical holomorphic chiral algebra of the purely bosonic sector of the twisted sigma-model on $X=S L(3) / B$. This observation is also consistent with the fact that $S(z)$ fails to correspond to a global section of the sheaf $\mathcal{O}_{X}^{c h}$ of CDO's - note that in our case, we actually have $S(z)=-3 T(z)$ in the classical theory, and this will mean that under quantum corrections to the action of $\bar{Q}_{+}$, we will have $\left[\bar{Q}_{+}, S_{z z}\right]=-3 \partial_{z}\left(R_{i \bar{j}} \partial_{z} \phi^{i} \psi^{\bar{j}}\right) \neq 0$ (since $R_{i \bar{j}} \neq 0$ for any flag manifold $S L(N) / B$ ), which corresponds in the Cech cohomology picture to the expression $\widetilde{\widehat{S}}(z)-\widehat{S}(z) \neq 0$, i.e., $\widehat{S}(z)$, the Cech cohomology counterpart to the $S(z)$ operator, will fail to be in $H^{0}\left(X, \widehat{\mathcal{O}}_{X}^{c h}\right)$. Consequently, one can always represent $S(z)$ by a classical $c$-number. This point will again be important when we discuss how one can define Hecke eigensheaves that will correspond to flat ${ }^{L} G$-bundles on a Riemann surface $\Sigma$ in our physical interpretation of the geometric Langlands correspondence for $G=S L(3)$.

The fact that $S(z)$ acts trivially in any OPE with other field operators implies that its Laurent modes will commute with the Laurent modes of any of these other field operators; in particular, they will commute with the Laurent modes of the $J_{a}(z)$ currents - in other words, the Laurent modes of $S(z)$ will span the centre $\mathfrak{z}\left(\widehat{\mathfrak{s l}}_{3}\right)$ of the completed universal enveloping algebra of the affine $S L(3)$ algebra $\widehat{\mathfrak{s l}}_{3}$ at the critical level $k=-3$ (generated by the Laurent modes of the $J_{a}(z)$ currents in the quantum chiral algebra of the twisted sigma-model on $S L(3) / B)$. Notice also that $S(z)$ is $\psi^{\bar{j}}$-independent and is therefore purely bosonic in nature. In other words, the local field $S(z)$ exists only in the classical holomorphic chiral algebra of the purely bosonic (or $\psi^{\bar{j}}$-independent) sector of the twisted sigma-model on $X=S L(3) / B$.

\section{A Classical Virasoro Algebra}

Note that since $S(z)$ is holomorphic in $z$ and is of conformal dimension two, one can expand it in terms of a Laurent expansion as

$$
S(z)=\sum_{n \in \mathbb{Z}} \hat{S}_{n} z^{-n-2}
$$

Recall that for the general case of $k \neq-3$, a quantum definition of $S(z)$ exists, such that the $\hat{S}_{n}$ modes of the Laurent expansion can be related to the $J_{a, n}$ modes of the $\widehat{\mathfrak{s l}}_{3}$ currents 
through the quantum commutator relations

$$
\begin{aligned}
{\left[\hat{S}_{n}, J_{a, m}\right] } & =-(k+3) m J_{a, n+m}, \\
{\left[\hat{S}_{n}, \hat{S}_{m}\right] } & =(k+3)\left((n-m) \hat{S}_{n+m}+\frac{8 k}{12}\left(n^{3}-n\right) \delta_{n,-m}\right),
\end{aligned}
$$

where $a=1,2, \ldots, 8$. If we now let $k=-3$, we will have $\left[\hat{S}_{n}, J_{a, m}\right]=\left[\hat{S}_{n}, \hat{S}_{m}\right]=0$ - the $S_{m}$ 's thus generate the (classical) centre of the completed universal enveloping algebra of $\widehat{\mathfrak{s l}}_{3}$ as mentioned above.

Since we now understand that $S(z)$ must be a holomorphic classical field at $k=-3$, let us rewrite the Laurent expansion of $S(z)$ as

$$
S(z)=\sum_{n \in \mathbb{Z}} S_{n} z^{-n-2}
$$

so as to differentiate the classical modes of expansion $S_{n}$ from their quantum counterpart $\hat{S}_{n}$ in (2.75). Unlike the $\hat{S}_{n}$ 's which obey the quantum commutator relations in (2.79) for an arbitrary level $k \neq-3$, the $S_{n}$ 's, being the modes of a Laurent expansion of a classical field, will instead obey Poisson bracket relations that define a certain classical algebra when $k=-3$.

Based on our arguments thus far, we learn that the quantum version of $S(z)$ as expressed in (2.75), must reduce to its classical counterpart as expressed in (2.80), when $k=-3$. In other words, one can see that by taking $(k+3) \rightarrow 0$, we are going to the classical limit. This is analogous to taking the $\hbar \rightarrow 0$ limit in any quantum mechanical theory whenever one wants to ascertain its classical counterpart. In fact, by identifying $(k+3)$ with $i \hbar$, and by noting that one must make the replacement from Possion brackets to commutators via $\left\{S_{n}, S_{m}\right\}_{P . B .} \rightarrow \frac{1}{i \hbar}\left[\hat{S}_{n}, \hat{S}_{m}\right]$ in quantising the $S_{n}$ 's into operators, we can ascertain the classical algebra generated by the $S_{n}$ 's from (2.79) as

$$
\left\{S_{n}, S_{m}\right\}_{P . B .}=(n-m) S_{n+m}-\frac{24}{12}\left(n^{3}-n\right) \delta_{n,-m}
$$

Since we have the classical relation $S(z) \sim T(z)$, it means that we can interpret the $S_{n}$ modes as the Virasoro modes of the Laurent expansion of the classical stress tensor field $T(z)$. In other words, the $S_{n}$ 's generate a classical Virasoro algebra with central charge -24 as given by (2.81). This can be denoted mathematically as the Virasoro Poisson algebra $\operatorname{Sym}^{\prime}\left(\operatorname{vir}_{-24}\right)$. 
A Higher-Spin Analog of the Segal-Sugawara Tensor and the Classical Holomorphic Chiral Algebra

For an affine $S L(N)$ algebra where $N>2$, one can generalise the Sugawara formalism to construct higher-spin analogs of the holomorphic stress tensor with the currents. These higher-spin analogs have conformal weights $3,4, \ldots N$. These higher-spin analogs are called Casimir operators, and were first constructed in [25].

In the context of our affine $S L(3)$ algebra with a module that is furnished by the global sections of the sheaf of CDO's on $X=S L(3) / B$, a spin-three analog of the holomorphic stress tensor will be given by the 3rd-order Casimir operator [12]

$$
T^{(3)}(z)=\frac{: \tilde{d}^{a b c}(k)\left(J_{a}\left(J_{b} J_{c}\right)\right)(z):}{k+3},
$$

where $\tilde{d}^{a b c}(k)$ is a completely symmetric traceless $\mathfrak{s l}_{3}$-invariant tensor of rank 3 that depends on the level $k$ of the affine $S L(3)$ algebra in question. $\tilde{d}^{a b c}(k)$ is also well-defined and finite at $k=-3$. The superscript on $T^{(3)}(z)$ just denotes that it is a spin-three analog of $T(z)$.

As with $T(z)$ in (2.74), $T^{(3)}(z)$ is ill-defined when $k=-3$. Nevertheless, one can always make reference to a higher-spin analog of the Segal-Sugawara tensor $S^{(3)}(z)$ that is well-defined for any finite value of $k$, where its relation to $T^{(3)}(z)$ is given by

$$
S^{(3)}(z)=(k+3) T^{(3)}(z),
$$

and

$$
S^{(3)}(z)=: \tilde{d}^{a b c}(k)\left(J_{a}\left(J_{b} J_{c}\right)\right)(z): .
$$

That is, the operator $S^{(3)}(z)$ generates in its OPE's with all other operators of the quantum theory, $(k+3)$ times the field transformations typically generated by $T^{(3)}(z)$.

Notice however, that at $k=-3, S^{(3)}(z)$ acts by zero in its OPE with any other operator. This is equivalent to saying that $T^{(3)}(z)$ does not exist as a quantum operator at all, since the only well-defined operator $S^{(3)}(z)$ which is supposed to generate the field transformations associated to $T^{(3)}(z)$, act by zero and thus generate no field transformations at all. From our $\bar{Q}_{+}$-Cech cohomology dictionary, this means that the $\psi^{\bar{i}}$-indepedent operator $T^{(3)}(z)$ will fail to correspond to a dimension three global section of $\widehat{\mathcal{O}}_{X}^{c h}$. Since we have, at the classical level, the relation $S^{(3)}(z)=-3 T^{(3)}(z)$, it will mean that $S^{(3)}(z)$ will also fail to correspond to a dimension three global section of $\widehat{\mathcal{O}}_{X}^{c h}$. Thus, $S^{(3)}(z)$ will fail to be an operator at the quantum level. Is it even a spin-three field in the classical holomorphic chiral algebra of 
the twisted sigma-model on $S L(3) / B$, one might ask. The answer is yes. To see this, recall that each of the $J_{a}(z)$ 's are separately $\bar{Q}_{+}$-invariant and not $\bar{Q}_{+}$-exact at the classical level. Therefore, the classical counterpart of $S^{(3)}(z)$ in (2.84) must also be such, which in turn means that it will be in the classical $\bar{Q}_{+}$-cohomology and hence classical chiral algebra of the twisted sigma-model on $S L(3) / B$.

The fact that $S^{(3)}(z)$ acts trivially in any OPE with other field operators implies that its Laurent modes will commute with the Laurent modes of any other operator; in particular, they will commute with the Laurent modes of the currents $J_{a}(z)$ for $a=1,2, \ldots, 8$ - in other words, the Laurent modes of $S^{(3)}(z)$ will span the centre $\mathfrak{z}\left(\widehat{\mathfrak{s l}}_{3}\right)$ of the completed universal enveloping algebra of the affine $S L(3)$ algebra $\widehat{\mathfrak{s l}}_{3}$ at the critical level $k=-3$ (generated by the Laurent modes of the $J_{a}(z)$ currents of the quantum chiral algebra of the twisted sigmamodel on $S L(3) / B)$. Last but not least, notice that the $S^{(3)}(z)$ field is also $\psi^{\bar{j}}$-independent and is therefore purely bosonic in nature. In other words, the local fields $S(z)$ and $S^{(3)}(z)$, whose Laurent modes together generate $\mathfrak{z}\left(\widehat{\mathfrak{s l}}_{3}\right)$, exist only in the classical holomorphic chiral algebra of the purely bosonic (or $\psi^{\bar{j}}$-independent) sector of the twisted sigma-model on $X=S L(3) / B$.

\section{A Classical $\mathcal{W}_{3}$-algebra}

For an affine $S L(3)$ algebra at an arbitrary level $k \neq-3$, as in the case of $S(z)$ discussed earlier, a quantum definition of $S^{(3)}(z)$ exists. In fact, consider the following operators given by $\bar{S}^{(3)}(z)=(\sqrt{3 / 200}): d^{a b c}\left(J_{a}\left(J_{b} J_{c}\right)\right)(z):$ and $\bar{S}(z)=(1 / 4): d^{a b} J_{a} J_{b}(z)$ :, where $d^{a b c}$ is just a rank-three extension of $d^{a b}$. It can be shown that $\bar{S}^{(3)}(z)$ and $\bar{S}(z)$ together span a closed Casimir OPE algebra which is isomorphic to a particular $\mathcal{W}_{3}$ OPE algebra [25]. This implies that for $k \neq-3$, both $\bar{S}^{(3)}(z)$ and $\bar{S}(z)$ and therefore $S(z) \sim$ : $d^{a b} J_{a} J_{b}(z)$ : and $S^{(3)}(z) \sim: d^{a b c}\left(J_{a}\left(J_{b} J_{c}\right)\right)(z)$ :, will exist as quantum operators in some cohomology the $\bar{Q}_{+}$-cohomology in this instance. This will in turn mean that $S(z)=(k+3) T(z)$ and $S^{(3)}(z)=(k+3) T^{(3)}(z)$ must also span a closed OPE algebra that is equivalent - at the level of $\bar{Q}_{+}$-cohomology - to this Casimir OPE algebra, when $k \neq-3$. Since we know that for $k \neq-3, T(z)$ will generate a Virasoro subalgebra of a closed $\mathcal{W}_{3}$ OPE algebra with central charge $c=8 k /(k+3)$, it will mean that $S(z)$ and $S^{(3)}(z)$ will satisfy a rescaled (by a factor of $(k+3))$ version of a closed $\mathcal{W}_{3}$ OPE algebra at $c=8 k /(k+3)$ for $k \neq-3$. Because $S^{(3)}(z)$ is holomorphic in $z$, we can Laurent expand it as

$$
S^{(3)}(z)=\sum_{n \in \mathbb{Z}} \hat{S}_{n}^{(3)} z^{-n-3}
$$


At $k \neq-3$, the Laurent modes $\hat{S}_{n}^{(3)}$, together with the Laurent modes $\hat{S}_{n}$ of $S(z)$, will then obey the following quantum commutator relations

$$
\left[\hat{S}_{n}, \hat{S}_{m}^{(3)}\right]=(k+3)(2 n-m) \hat{S}_{n+m}^{(3)}
$$

and

$$
\begin{aligned}
& {\left[\hat{S}_{m}^{(3)}, \hat{S}_{n}^{(3)}\right]=(k+3)\left[\frac{8 k}{360} m\left(m^{2}-1\right)\left(m^{2}-4\right) \delta_{m,-n}\right]} \\
& +(k+3)\left[(m-n)\left(\frac{1}{15}(m+n+3)(m+n+2)-\frac{1}{6}(m+2)(n+2)\right) \hat{S}_{m+n}\right] \\
& +(k+3)\left[\frac{16}{62 k+66}(m-n)\left(\sum_{p} \hat{S}_{m+n-p} \hat{S}_{p}-\frac{3}{10}(k+3)(m+n+3)(m+n+2) \hat{S}_{m+n}\right)\right] .
\end{aligned}
$$

Now let us consider the case when $k=-3$. From our earlier explanations about the nature of $S^{(3)}(z)$ and $S(z)$ at $k=-3$, we find that they will cease to exist as quantum operators at $k=-3$. Since we understand that $S^{(3)}(z)$, just like $S(z)$, must be a holomorphic classical field at $k=-3$, we shall rewrite the Laurent expansion of $S^{(3)}(z)$ as

$$
S^{(3)}(z)=\sum_{n \in \mathbb{Z}} S_{n}^{(3)} z^{-n-3}
$$

so as to differentiate the classical modes of expansion $S_{n}^{(3)}$ from their quantum counterpart $\hat{S}_{n}^{(3)}$ in (2.85). Unlike the $\hat{S}_{n}^{(3)}$ 's which obey the quantum commutator relations in (2.87) for an arbitrary level $k \neq-3$, the $S_{n}^{(3)}$ 's, being the modes of a Laurent expansion of a classical field, will instead obey Poisson bracket relations that define a certain classical algebra when $k=-3$. Since every $\hat{S}_{n}^{(3)}$ must reduce to its classical counterpart $S_{n}^{(3)}$ when $k=-3$, one can see that by taking $(k+3) \rightarrow 0$, we are actually going to the classical limit. This is analogous to taking the $\hbar \rightarrow 0$ limit in any quantum mechanical theory whenever one wants to ascertain its classical counterpart. In fact, by identifying $(k+3)$ with $i \hbar$, and by noting that one must make the replacement from Possion brackets to commutators via $\left\{D_{n}, D_{m}\right\}_{P . B .} \rightarrow \frac{1}{i \hbar}\left[\hat{D}_{n}, \hat{D}_{m}\right]$ in quantising any classical mode $D_{n}$ into an operator, we can ascertain the classical algebra generated by the $S_{n}^{(3)}$ 's and $S_{n}$ 's from (2.86) and (2.87) as

$$
\left\{S_{n}, S_{m}^{(3)}\right\}_{P . B .}=(2 n-m) \hat{S}_{n+m}^{(3)}
$$


and

$$
\begin{aligned}
\left\{S_{m}^{(3)}, S_{n}^{(3)}\right\}_{P . B .}= & -\frac{24}{360} m\left(m^{2}-1\right)\left(m^{2}-4\right) \delta_{m,-n}-\frac{4}{30}(m-n) \sum_{p} S_{m+n-p} S_{p} \\
& +(m-n)\left(\frac{1}{15}(m+n+3)(m+n+2)-\frac{1}{6}(m+2)(n+2)\right) S_{m+n} .
\end{aligned}
$$

Together with the earlier expression

$$
\left\{S_{n}, S_{m}\right\}_{P . B .}=(n-m) S_{n+m}-\frac{24}{12}\left(n^{3}-n\right) \delta_{n,-m}
$$

we see that the $S_{m}^{(3)}$ 's and $S_{m}$ 's generate a classical $\mathcal{W}_{3}$-algebra with central charge -24 . Note that the algebra is closed amongst the $S_{n}^{(3)}$ and $S_{n}$ modes; this is true because both $S^{(3)}(z)$ and $S(z)$ are in the classical $\bar{Q}_{+}$-cohomology of the sigma-model 8 Thus, if we denote this classical algebra by $\mathcal{W}_{3}(-24)$, we then have the identification $\mathfrak{z}\left(\widehat{\mathfrak{s l}}_{3}\right) \simeq \mathcal{W}_{3}(-24)$.

\subsection{A Gauged WZW Model and the Geometric Langlands Correspondence for $G=S L(3)$}

\section{The B-gauged WZW Model on SL(3)}

According to our discussion in $§ 2.2$, the classical holomorphic chiral algebra of the purely bosonic sector of the twisted sigma-model on $S L(3) / B$ - in which lie the fields $S(z)$ and $S^{(3)}(z)$ - will be given by the classical, holomorphic BRST-cohomology of a $B$-gauged WZW model on $S L(3)$ - from which one ought to find non-trivial classes that are in one-toone correspondence with the fields $S(z)$ and $S^{(3)}(z)$ respectively. As such, we shall proceed to specialise the action $S_{\text {B-gauged }}\left(g, A_{z}, A_{\bar{z}}, J^{+}, \bar{J}^{+}\right)$of a (non-dynamically) $B$-gauged WZW model on any $S L(N)$ defined in (2.43) of $\S 2.2$, to the case where the target-space is now $S L(3)$.

In the case of $S L(3)$, we have $\operatorname{dim} \mathfrak{n}_{ \pm}=3$ and $\operatorname{dim} \mathfrak{c}=2$, so we can write $J(z)=$ $\sum_{a=1}^{3} J_{-}^{a}(z) t_{a}^{-}+\sum_{a=1}^{2} J_{c}^{a}(z) t_{a}^{c}+\sum_{l=1}^{3} J_{+}^{l}(z) t_{l}^{+}$, and $\bar{J}(\bar{z})=\sum_{a=1}^{3} \bar{J}_{-}^{a}(\bar{z}) t_{a}^{-}+\sum_{a=1}^{2} \bar{J}_{c}^{a}(\bar{z}) t_{a}^{c}+$ $\sum_{l=1}^{3} \bar{J}_{+}^{l}(\bar{z}) t_{l}^{+}$, where $t_{a}^{-} \in \mathfrak{n}_{-}, t_{a}^{c} \in \mathfrak{c}$, and $t_{a}^{+} \in \mathfrak{n}_{+}$. One can also write $M=\sum_{a=1}^{3} M_{-}^{a} t_{a}^{-}+$

\footnotetext{
${ }^{8}$ Note at this point that if $\mathcal{O}$ and $\mathcal{O}^{\prime}$ are non-exact $\bar{Q}_{+}$-closed observables in the (classical) $\bar{Q}_{+}$-cohomology, i.e., $\left\{\bar{Q}_{+}, \mathcal{O}\right\}=\left\{\bar{Q}_{+}, \mathcal{O}^{\prime}\right\}=0$, then $\left\{\bar{Q}_{+}, \mathcal{O O}^{\prime}\right\}=0$. Moreover, if $\left\{\bar{Q}_{+}, \mathcal{O}\right\}=0$, then $\mathcal{O}\left\{\bar{Q}_{+}, W\right\}=$ $\left\{\bar{Q}_{+}, \mathcal{O} W\right\}$ for any observable $W$. These two statements mean that the cohomology classes of observables that commute with $\bar{Q}_{+}$form a closed and well-defined (classical) algebra.
} 
$\sum_{a=1}^{2} M_{c}^{a} t_{a}^{c}+\sum_{l=1}^{3} M_{+}^{l} t_{l}^{+}$, where $M_{-; c}^{a}$ and $M_{+}^{l}$ are arbitrary number constants, and $\bar{M}=$ $\sum_{a=1}^{3} \bar{M}_{-}^{a} t_{a}^{-}+\sum_{a=1}^{2} \bar{M}_{c}^{a} t_{a}^{c}+\sum_{l=1}^{3} \bar{M}_{+}^{l} t_{l}^{+}$, where $\bar{M}_{-; c}^{a}$ and $\bar{M}_{+}^{l}$ are arbitrary number constants. In addition, one can also write $A_{\bar{z}}=\sum_{l=1}^{3} \tilde{A}_{\bar{z}}^{l} t_{l}^{+}$and $A_{z}=\sum_{l=1}^{3} \tilde{A}_{z}^{l} t_{l}^{+}$. Let us denote $J^{+}(z)=\sum_{l=1}^{3} J_{+}^{l}(z) t_{l}^{+}$and $M^{+}=\sum_{l=1}^{3} M_{+}^{l} t_{l}^{+}$. Let us also denote $\bar{J}^{+}(\bar{z})=\sum_{l=1}^{3} \bar{J}_{+}^{l}(\bar{z}) t_{l}^{+}$ and $\bar{M}^{+}=\sum_{l=1}^{3} \bar{M}_{+}^{l} t_{l}^{+}$. Hence, one can write the action for the $B$-gauged WZW model on $S L(3)$ as

$$
\begin{gathered}
S_{S L(3)}\left(g, A_{z}, A_{\bar{z}}, J^{+}, \bar{J}^{+}\right)=S_{\mathrm{WZ}}(g)-\frac{k^{\prime}}{2 \pi} \int_{\Sigma} d^{2} z \sum_{l=1}^{3}\left[\tilde{A}_{\bar{z}}^{l}\left(J_{+}^{l}(z)+M_{+}^{l}\right)-\tilde{A}_{z}^{l}\left(\bar{J}_{+}^{l}(\bar{z})+\bar{M}_{+}^{l}\right)\right] \\
-\operatorname{Tr}\left[A_{z} g A_{\bar{z}} g^{-1}-A_{z} A_{\bar{z}}\right]
\end{gathered}
$$

Due to the $B$-gauge invariance of the theory, we must divide the measure in any path integral computation by the volume of the $B$-gauge symmetry. That is, the partition function has to take the form

$$
Z_{S L(3)}=\int_{\Sigma} \frac{\left[g^{-1} d g, d \tilde{A}_{z}^{l}, d \tilde{A}_{\bar{z}}^{l}\right]}{\text { (gauge volume) }} \exp \left(i S_{S L(3)}\left(g, A_{z}, A_{\bar{z}}, J^{+}, \bar{J}^{+}\right)\right)
$$

One must now fix this gauge invariance to eliminate the non-unique degrees of freedom. One can do this by employing the BRST formalism which requires the introduction of FaddevPopov ghost fields.

In order to obtain the holomorphic BRST transformations of the fields, one simply replaces the position-dependent infinitesimal gauge parameter $\epsilon^{l}$ of $h=B=\exp \left(-\sum_{l=1}^{3} \epsilon^{l} t_{l}^{+}\right)$ in the corresponding left-sector of the gauge transformations in (2.34) with the ghost field $c^{l}$, which then gives us

$$
\delta_{\mathrm{BRST}}(g)=-c^{l} t_{l}^{+} g, \quad \delta_{\mathrm{BRST}}\left(\tilde{A}_{\bar{z}}^{l}\right)=-D_{\bar{z}} c^{l}, \quad \delta_{\mathrm{BRST}}(\text { others })=0 .
$$

The components of the ghost field $c(z)=\sum_{l=1}^{3} c^{l}(z) t_{l}^{+}$and those of its anti-ghost partner $b(z)=\sum_{l=1}^{3} b^{l}(z) t_{l}^{+}$will transform as

$$
\delta_{\mathrm{BRST}}\left(c^{l}\right)=-\frac{1}{2} f_{m k}^{l} c^{m} c^{k}, \quad \delta_{\mathrm{BRST}}\left(b^{l}\right)=\tilde{B}^{l}, \quad \delta_{\mathrm{BRST}}\left(\tilde{B}^{l}\right)=0,
$$

where the $f_{m k}^{l}$ 's are the structure constants of the nilpotent subalgebra $\mathfrak{n}_{+}$. Also, the $\tilde{B}^{l}$ 's are the Nakanishi-Lautrup auxiliary fields that are the BRST transforms of the $b^{l}$ 's. They also serve as a Lagrange multipliers to impose the gauge-fixing conditions. 
In order to obtain the antiholomorphic BRST transformations of the fields, one employs the same recipe to the corresponding right-sector of the gauge transformations in (2.34) with the infinitesimal position-dependent gauge parameter now replaced by the ghost field $\bar{c}^{l}$, which then gives us

$$
\bar{\delta}_{\mathrm{BRST}}(g)=\bar{c}^{l} t_{l}^{+} g, \quad \bar{\delta}_{\mathrm{BRST}}\left(\tilde{A}_{z}^{l}\right)=-D_{z} \bar{c}^{l}, \quad \bar{\delta}_{\mathrm{BRST}}(\text { others })=0
$$

The components of the ghost field $\bar{c}(\bar{z})=\sum_{l=1}^{3} \bar{c}^{l}(\bar{z}) t_{l}^{+}$and those of its anti-ghost partner $\bar{b}(\bar{z})=\sum_{l=1}^{3} \bar{b}^{l}(\bar{z}) t_{l}^{+}$will transform as

$$
\bar{\delta}_{\mathrm{BRST}}\left(\bar{c}^{l}\right)=-\frac{1}{2} f_{m k}^{l} \bar{c}^{m} \bar{c}^{k}, \quad \bar{\delta}_{\mathrm{BRST}}\left(\bar{b}^{l}\right)=\tilde{\bar{B}}^{l}, \quad \bar{\delta}_{\mathrm{BRST}}\left(\tilde{\bar{B}}^{l}\right)=0 .
$$

In the above, the $\tilde{\bar{B}}^{l}$ 's are the Nakanishi-Lautrup auxiliary fields that are the antiholomorphic BRST transforms of the $\bar{b}^{l}$ fields. They also serve as Lagrange multipliers to impose the gauge-fixing conditions.

Since the BRST transformations in (2.94) and (2.96) are just infinitesimal versions of the gauge transformations in (2.34), $S_{S L(3)}\left(g, A_{z}, A_{\bar{z}}, J^{+}, \bar{J}^{+}\right)$will be invariant under them. An important point to note at this juncture is that in addition to $\left(\delta_{\mathrm{BRST}}+\bar{\delta}_{\mathrm{BRST}}\right) \cdot\left(\delta_{\mathrm{BRST}}+\right.$ $\left.\bar{\delta}_{\mathrm{BRST}}\right)=0$, the holomorphic and antiholomorphic BRST-variations are also separately nilpotent, i.e., $\delta_{\mathrm{BRST}}^{2}=0$ and $\bar{\delta}_{\mathrm{BRST}}^{2}=0$. Moreover, $\delta_{\mathrm{BRST}} \cdot \bar{\delta}_{\mathrm{BRST}}=-\bar{\delta}_{\mathrm{BRST}} \cdot \delta_{\mathrm{BRST}}$. This means that the BRST-cohomology of the $B$-gauged WZW model on $S L(3)$ can be decomposed into independent holomorphic and antiholomorphic sectors that are just complex conjugate of each other, and that it can be computed via a spectral sequence, whereby the first two complexes will be furnished by its holomorphic and antiholomorphic BRST-cohomologies respectively. Since we will only be interested in the holomorphic chiral algebra of the $B$ gauged WZW model on $S L(3)$ (which as mentioned, is just identical to its antiholomorphic chiral algebra by a complex conjugation), we shall henceforth focus on the holomorphic BRST-cohomology of the $B$-gauged WZW model on $S L(3)$.

By the usual recipe of the BRST formalism, one can fix the gauge by adding to the BRST-invariant action $S_{S L(3)}\left(g, A_{z}, A_{\bar{z}}, J^{+}, \bar{J}^{+}\right)$, a BRST-exact term. Since the BRST transformation by $\left(\delta_{\mathrm{BRST}}+\bar{\delta}_{\mathrm{BRST}}\right)$ is nilpotent, the new total action will still be BRST-invariant as required. The choice of the BRST-exact operator will then define the gauge-fixing conditions. A consistent choice of the BRST-exact operator that will give us the requisite action 
for the ghost and anti-ghost fields is

$$
S_{S L(3)}\left(g, A_{z}, A_{\bar{z}}, J^{+}, \bar{J}^{+}\right)+\left(\delta_{\mathrm{BRST}}+\bar{\delta}_{\mathrm{BRST}}\right)\left(\frac{k^{\prime}}{2 \pi} \int_{\Sigma} d^{2} z \sum_{l=1}^{3} \tilde{A}_{\bar{z}}^{l} b^{l}+\tilde{A}_{z}^{l} \bar{b}^{l}\right)
$$

where one will indeed have the desired total action, which can be written as

$$
\begin{array}{r}
S_{\mathrm{WZW}}(g)-\frac{k^{\prime}}{2 \pi} \int_{\Sigma} d^{2} z\left\{\sum_{l=1}^{3}\left[\tilde{A}_{\bar{z}}^{l}\left(J_{+}^{l}(z)+M_{+}^{l}-\tilde{B}^{l}\right)-\tilde{A}_{z}^{l}\left(\bar{J}_{+}^{l}(\bar{z})+\bar{M}_{+}^{l}+\tilde{\bar{B}}^{l}\right)\right]\right. \\
\left.-\operatorname{Tr}\left[A_{z} g A_{\bar{z}} g^{-1}-A_{z} A_{\bar{z}}\right]\right\}+\frac{k^{\prime}}{2 \pi} \int_{\Sigma} d^{2} z \sum_{l=1}^{3}\left(c^{l} D_{\bar{z}} b^{l}++\bar{c}^{l} D_{z} \bar{b}^{l}\right) .
\end{array}
$$

From the equations of motion by varying the $\tilde{B}^{l}$ 's, we have the conditions $\tilde{A}_{\bar{z}}^{l}=0$ for $l=1,2,3$. From the equations of motion by varying the $\tilde{\bar{B}}^{l}$ 's, we also have the conditions $\tilde{A}_{z}^{l}=0$ for $l=1,2,3$. Thus, the partition function of the $B$-gauged WZW model can also be expressed as

$$
Z_{S L(3)}=\int\left[g^{-1} d g, d b, d c, d \bar{b}, d \bar{c}\right] \exp \left(i S_{\mathrm{WZW}}(g)+\frac{i k^{\prime}}{2 \pi} \int_{\Sigma} d^{2} z \operatorname{Tr}\left(c \cdot \partial_{\bar{z}} b\right)(z)+\operatorname{Tr}\left(\bar{c} \cdot \partial_{z} \bar{b}\right)(\bar{z})\right)
$$

where the holomorphic BRST variations of the fields which leave the effective action in (2.99) invariant are now given by

$$
\begin{aligned}
& \delta_{\mathrm{BRST}}(g)=-c^{m} t_{m}^{+} g, \quad \delta_{\mathrm{BRST}}\left(c^{l}\right)=-\frac{1}{2} f_{m k}^{l} c^{m} c^{k}, \quad \delta_{\mathrm{BRST}}\left(b^{l}\right)=J_{+}^{l}+M_{+}^{l}-f_{m k}^{l} b^{m} c^{k}, \\
& \delta_{\mathrm{BRST}}(\text { others })=0 .
\end{aligned}
$$

Though we did not make this obvious in our discussion above, by integrating out the $\tilde{A}_{\bar{z}}^{l}$ 's in (2.92), and using the above conditions $\tilde{A}_{z}^{l}=0$ for $l=1,2,3$, we find that we actually have the relations $\left(J_{+}^{l}(z)+M_{+}^{l}\right)=0$ for $l=1,2,3$. These relations (involving the current associated to the Borel subalgebra $\mathfrak{b}$ of the group $B$ that we are modding out by) will lead us directly to the correct form of the holomorphic stress tensor for the gauged WZW model without reference to a coset formalism. Let us look at this more closely. Since we have, in the holomorphic BRST-cohomology of this non-dynamically $B$-gauged WZW model on $S L(3)$, currents that generate an affine $S L(3)$ OPE algebra, (consistent with the presence of an affine $S L(3)$ OPE algebra generated by operators in the holomorphic chiral algebra of the purely bosonic sector of the sigma-model on $S L(3) / B)$, we can employ the Sugawara 
formalism to construct the holomorphic stress tensor associated to the $S_{\mathrm{WZW}}(g)$ part of the total action from the currents, and it can be written as

$$
T_{S L(3)}(z)=\frac{: J(z) \cdot J(z):}{\left(k^{\prime}+3\right)}
$$

where the above dot product between the currents $J(z)$ is taken with respect to the CartanKilling metric on $\mathfrak{s l}_{3}$. Note that with respect to $T_{S L(3)}(z)$, the currents $J_{+}^{l}(z)$ for $l=1,2,3$ (and in fact all the other affine $S L(3)$ currents) have conformal dimension one. This is inconsistent with the condition $J_{+}^{l}(z)=-M_{+}^{l}$, as the $M_{+}^{l}$ 's are constants of conformal dimension zero. This means that one must modify $T_{S L(3)}(z)$ so that the $J_{+}^{l}(z)$ 's will have conformal dimension zero. A physically consistent modification involves adding to $T_{S L(3)}(z)$ a term that has conformal dimension two. A little thought will reveal that the only consistent candidate for the modified stress tensor of $S_{\mathrm{WZW}}(g)$ will be given by

$$
T_{\text {modified }}(z)=T_{S L(3)}(z)+\partial_{z} J_{c}^{1}(z)+\partial_{z} J_{c}^{2}(z)
$$

With respect to $T_{\text {modified }}(z)$, the currents $J_{+}^{1}(z)$ and $J_{+}^{2}(z)$ have vanishing conformal dimensions as required. On the other hand, the current $J_{+}^{3}(z)$ will now have conformal dimension -1 . Thus, it must mean that there cannot be any restriction on the conformal dimension of $J_{+}^{3}(z)$, and therefore, $M_{+}^{3}$ must vanish.

In order for the above observations to be consistent with the fact that the BRST-charge $Q_{\mathrm{BRST}}$ generating the variations $\delta_{\mathrm{BRST}}\left(b^{l}\right)$ of (2.100) must be a scalar of conformal dimension zero, we find that $b^{l}$ 's and hence the $c^{l}$ 's must have the following conformal dimensions: $\left(b^{1}, b^{2}, b^{3}\right) \leftrightarrow(0,0,-1)$ and $\left(c^{1}, c^{2}, c^{3}\right) \leftrightarrow(1,1,2)$. From the effective action in (2.99), we can compute the holomorphic stress tensor of the left-moving ghost/anti-ghost system. Including this contribution, we find that the total holomorphic stress tensor can be written as

$$
\begin{aligned}
T_{\text {total }}(z)= & T_{S L(3)}(z)+\partial_{z} J_{c}^{1}(z)+\partial_{z} J_{c}^{2}(z)+\partial_{z} b^{1}(z) c_{z}^{1}(z)+\partial_{z} b^{2}(z) c_{z}^{2}(z) \\
& +2 \partial_{z} b^{3, z}(z) c_{z z}^{3}(z)+b^{3, z}(z) \partial_{z} c_{z z}^{3}(z) .
\end{aligned}
$$

The conserved current associated to the holomorphic BRST-variations of the fields in (2.100) can be computed as

$$
I_{\mathrm{BRST}}=c_{z}^{1}\left(J_{+}^{1}(z)+M_{+}^{1}\right)+c_{z}^{2}\left(J_{+}^{2}(z)+M_{+}^{2}\right)+c_{z z}^{3} J_{+}^{3, z}(z)+c_{z}^{1}(z) c_{z}^{2}(z) b^{3, z}(z) .
$$


Apart from a trivial inspection, one can also verify, from the affine $S L(3)$ OPE algebra and the OPE algebra between the left-moving ghost/anti-ghost fields, that $I_{\mathrm{BRST}}$ is of conformal dimension one with respect to $T_{\text {total }}(z)$. This means that as required, one can define a conformal dimension zero scalar BRST-charge

$$
Q_{\mathrm{BRST}}=\oint \frac{d z}{2 \pi i} c_{z}^{1}\left(J_{+}^{1}(z)+M_{+}^{1}\right)+c_{z}^{2}\left(J_{+}^{2}(z)+M_{+}^{2}\right)+c_{z z}^{3} J_{+}^{3, z}(z)+c_{z}^{1}(z) c_{z}^{2}(z) b^{3, z}(z),
$$

which generates the correct holomorphic BRST-variation of the fields. Note that the holomorphic BRST-charge can also be written in its general form as

$$
Q_{\mathrm{BRST}}=\oint \frac{d z}{2 \pi i}\left(\sum_{l=1}^{\mathrm{dimn}_{+}=3} c^{l}\left(J_{+}^{l}(z)+M_{+}^{l}\right)-\frac{1}{2} \sum_{l, m, k=1}^{\mathrm{dim} \mathfrak{n}_{+}=3} f_{m k}^{l} b^{m} c^{l} c^{k}\right) .
$$

Using the free field OPE's that the ghost fields generate, one can immediately verify that $Q_{\text {BRST }}$ as given in (2.106) will indeed generate the field variations in (2.100).

\section{More about the Holomorphic Stress Tensor and its Higher-Spin Analog}

Since $I_{\mathrm{BRST}}$ is of conformal dimension one with respect to $T_{\text {total }}(z)$, it will mean that $T_{\text {total }}(z)$ will be annihilated by $Q_{\mathrm{BRST}}$. Moreover, one can also check that $T_{\text {total }}(z) \neq$ $\left\{Q_{\mathrm{BRST}}, \cdots\right\}$. In other words, $T_{\text {total }}(z)$ is a spin-two observable in the holomorphic BRSTcohomology of the $B$-gauged WZW model on $S L(3)$. From the explicit expression of $T_{\text {total }}(z)$, we find that its Laurent modes will generate a Virasoro algebra with central charge $c=2-24\left(k^{\prime}+2\right)^{2} /\left(k^{\prime}+3\right)$.

Note that one can define a cohomologically equivalent total holomorphic stress tensor $T_{W_{3}}(z)$ via

$$
T_{W_{3}}(z)=T_{\text {total }}(z)+\left\{Q_{\mathrm{BRST}}, t(z)\right\}
$$

whereby

$$
\begin{aligned}
t(z)= & \gamma_{1, z}(z) \partial_{z} b^{1}(z)+\gamma_{2, z}(z) \partial_{z} b^{2}(z)+2 \gamma_{3, z z}(z) \partial_{z} b^{3, z}(z)+\partial_{z} \gamma_{3, z z}(z) b^{3, z}(z) \\
& -\gamma_{1, z}(z) \partial_{z}\left[\gamma_{2, z}(z) b^{3, z}(z)\right]
\end{aligned}
$$

such that

$$
T_{W_{3}}(z)=-\frac{1}{2}\left(\left(\partial_{z} \varphi_{1}(z)\right)^{2}+\left(\partial_{z} \varphi_{2}(z)\right)^{2}+\frac{i 2\left(k^{\prime}+2\right)}{\sqrt{2 k^{\prime}+6}} \partial_{z}^{2} \varphi_{1}(z)\right),
$$

in which the $\gamma$ 's and $\varphi$ 's are just auxillary fields which satisfy the OPE's of a free $\beta \gamma$ and free scalar system respectively. It can be shown [26] that $T_{W_{3}}(z)$, together with a spin-three field 
$T_{W_{3}}^{(3)}(z)$ which is a higher-spin analog of $T_{W_{3}}(z)$, will satisfy the free boson realisation of a closed $\mathcal{W}_{3}$ OPE algebra with the same central charge $c=2-24\left(k^{\prime}+2\right)^{2} /\left(k^{\prime}+3\right)=50-24\left(k^{\prime}+\right.$ $3)-24 /\left(k^{\prime}+3\right)$. This implies that one can always find a spin-three observable, independent of the $\gamma^{\prime}$ 's and $\varphi$ 's, and composed out of the fields in the gauged WZW model only, which is cohomologically equivalent to $T_{W_{3}}^{(3)}(z)$, and which is also non-trivial in the holomorphic BRST-cohomology of the gauged WZW model.9 Let us denote this spin-three observable as $T_{\text {total }}^{(3)}(z)$. Note that $T_{\text {total }}^{(3)}(z)$ is just a spin-three analog of $T_{\text {total }}(z)$, and together with $T_{\text {total }}(z)$, it will generate a $\mathcal{W}_{3}$ OPE algebra with central charge $c=50-24\left(k^{\prime}+3\right)-24 /\left(k^{\prime}+3\right)$ for finite (and therefore non-classical) values of the level $k^{\prime}$. This observation will turn out to be consistent with our discussion in $\S 3.3$ where we unravel the role that the $B$-gauged WZW model on $S L(3)$ plays in a physical realisation of the DS-reduction scheme of generating a $\mathcal{W}_{k^{\prime}}\left(\widehat{\mathfrak{s l}}_{3}\right)$ OPE algebra.

Last but not least, a soon-to-be relevant point to note is that since quantum corrections can only annihilate classes in the BRST-cohomology and not create them, the classical counterparts of the holomorphic stress tensor $T_{\text {total }}(z)$ and its spin-three analog $T^{(3)}(z)$, will be the spin-two and spin-three fields $T_{\text {classical }}(z)$ and $T_{\text {classical }}^{(3)}(z)$ which lie in the classical, holomorphic BRST-cohomology (or holomorphic chiral algebra) of the $B$-gauged WZW model on $S L(3)$, where $T_{\text {classical }}(z)$ will generate a classical Virasoro transformation on the fields, while $T_{\text {classical }}^{(3)}(z)$ will generate a classical $\mathcal{W}_{3}$ transformation on them.

A Duality of Classical $\mathcal{W}$-Algebras Underlying a Geometric Langlands Correspondence for $G=S L(3)$

Recall that the observables in the holomorphic BRST-cohomology of the gauged WZW model that should correspond to $S(z)$ and $S^{(3)}(z)$ of the holomorphic chiral algebra of the purely bosonic, $\psi^{\bar{j}}$-independent sector of the twisted sigma-model on $S L(3) / B$, must have the same spins as $S(z)$ and $S^{(3)}(z)$. Since $S(z)$ generates a classical Virasoro symmetry on the worldsheet, it should correspond to a spin-two observable in the classical, holomorphic BRST-cohomology of the $B$-gauged WZW model on $S L(3)$ which generates a classical Virasoro symmetry on the worldsheet. Since $S^{(3)}(z)$ generates a classical $\mathcal{W}_{3}$ symmetry on the worldsheet, it should correspond to a spin-three observable in the classical, holomorphic BRST-cohomology of the $B$-gauged WZW model on $S L(3)$ which generates a classical $\mathcal{W}_{3}$

\footnotetext{
${ }^{9}$ Note that within the $\mathcal{W}_{3}$ OPE algebra, we have an OPE of the form $T_{W_{3}}^{(3)}(z) \cdot T_{W_{3}}^{(3)}\left(z^{\prime}\right)$, which is equivalent, up to singular terms, to a sum of the fields $T_{W_{3}}(z)$ and its partial derivatives only. These terms in the sum are certainly BRST-closed but non-exact. Thus, in order to be consistent with the OPE, it implies that $T_{W_{3}}^{(3)}(z)$ must also be BRST-closed and non-exact.
} 
symmetry on the worldsheet.

In order to ascertain the classical observable which corresponds to $S(z)$, first recall that the quantum definition of $S(z)$ at $k \neq-3$ is given by $S(z)=(k+3) T(z)$. Notice that since the stress tensor $T(z)$ also exists in the holomorphic chiral algebra of the purely bosonic, $\psi^{\bar{j}_{-}}$ independent sector of the sigma-model on $S L(3) / B$, it will imply that $T(z)$ must correspond to the stress tensor $T_{\text {total }}(z)$ of the $B$-gauged WZW model on $S L(3)$. Thus, at $k \neq-3$, $S(z)$ will correspond to $\bar{T}_{\text {total }}(z)=(k+3) T_{\text {total }}(z)$, and at $k=-3, S(z)$ will correspond to the classical counterpart $\bar{T}_{\text {classical }}(z)$ of $\bar{T}_{\text {total }}(z)$. Note that $\bar{T}_{\text {classical }}(z)$ lies in the classical, holomorphic BRST-cohomology of the $B$-gauged WZW model on $S L(3)$ as required - at $k=-3, \bar{T}_{\text {total }}(z)$, which usually exists as a quantum operator, will act by zero in its OPE's with any other operator, i.e., it will reduce to its classical counterpart $\bar{T}_{\text {classical }}(z)$ in the classical, holomorphic BRST-cohomology of the gauged WZW model. Moreover, since the shift in 3 in the factor $(k+3)$ is due to a quantum effect as explained earlier, $S(z)$ will actually correspond to $\bar{T}_{\text {classical }}(z)=-3 T_{\text {classical }}(z)$ at $k=-3$. Hence, $S(z)$ will indeed correspond to a spin-two field in the classical, holomorphic BRST-cohomology of the $B$-gauged WZW on $S L(3)$ which generates a classical Virasoro transformation of the fields.

In order to ascertain the classical observable which corresponds to $S^{(3)}(z)$, first recall that the quantum definition of $S^{(3)}(z)$ at $k \neq-3$ is given by $S^{(3)}(z)=(k+3) T^{(3)}(z)$. Notice that since $T^{(3)}(z)$ also exists as a spin-three analog of $T(z)$ in the holomorphic chiral algebra of the purely bosonic, $\psi^{\bar{j}}$-independent sector of the sigma-model on $S L(3) / B$, it will imply that $T^{(3)}(z)$ must correspond to the spin-three operator $T_{\text {total }}(z)$ of the $B$-gauged WZW model on $S L(3)$. Thus, at $k \neq-3, S^{(3)}(z)$ will correspond to $\bar{T}_{\text {total }}^{(3)}(z)=(k+3) T_{\text {total }}^{(3)}(z)$, and at $k=-3, S(z)$ will correspond to the classical counterpart $\bar{T}_{\text {classical }}^{(3)}(z)$ of $\bar{T}_{\text {total }}^{(3)}(z)$. Note that $\bar{T}_{\text {classical }}^{(3)}(z)$ lies in the classical, holomorphic BRST-cohomology of the B-gauged WZW model on $S L(3)$ as required - at $k=-3, \bar{T}_{\text {total }}^{(3)}(z)$, which usually exists as a quantum operator, will act by zero in its OPE's with any other operator, i.e., it will reduce to its classical counterpart $\bar{T}_{\text {classical }}^{(3)}(z)$ in the classical, holomorphic BRST-cohomology of the gauged WZW model. Moreover, since the shift in 3 in the factor $(k+3)$ is due to a quantum effect as explained earlier, $S^{(3)}(z)$ will actually correspond to $\bar{T}_{\text {classical }}^{(3)}(z)=-3 T_{\text {classical }}^{(3)}(z)$ at $k=-3$. Hence, $S^{(3)}(z)$ will indeed correspond to a spin-three field in the classical, holomorphic BRSTcohomology of the $B$-gauged WZW on $S L(3)$ which generates a classical $\mathcal{W}_{3}$ transformation of the fields.

What is the classical algebra generated by the Laurent modes of $\bar{T}_{\text {classical }}(z)$ ? To ascertain this, first recall that the Laurent modes of $T_{\text {total }}(z)$ generate a Virasoro algebra of central 
charge $c=50-24\left(k^{\prime}+3\right)-24 /\left(k^{\prime}+3\right)$. Hence, the Virasoro modes of $T_{\text {total }}(z)=\sum_{n} \hat{L}_{n} z^{-n-2}$ will obey the following quantum commutator relation

$$
\left[\hat{L}_{n}, \hat{L}_{m}\right]=(n-m) \hat{L}_{n+m}+\frac{1}{12}\left[50-24\left(k^{\prime}+3\right)-24 /\left(k^{\prime}+3\right)\right]\left(n^{3}-n\right) \delta_{n,-m} .
$$

Therefore, the commutator relations involving the $\hat{\bar{L}}_{n}$ modes of $\bar{T}_{\text {total }}(z)=\sum_{n} \hat{\bar{L}}_{n} z^{-n-2}$ will be given by

$\left[\hat{\bar{L}}_{n}, \hat{\bar{L}}_{m}\right]=(k+3)\left[(n-m) \hat{\bar{L}}_{n+m}+\left(n^{3}-n\right) \delta_{n,-m}\left(\frac{50}{12}(k+3)-\frac{24(k+3)\left(k^{\prime}+3\right)}{12}-\frac{2(k+3)}{\left(k^{\prime}+3\right)}\right)\right]$.

At $k=-3, \bar{T}_{\text {total }}(z)$ will cease to have a quantum definition, and it will reduce to its classical counterpart $\bar{T}_{\text {classical }}(z)$. Hence, the $k \rightarrow-3$ (and $k^{\prime} \rightarrow \infty$ ) limit of the commutator relation in (2.111), can be interpreted as its classical limit. Therefore, one can view the term $(k+3)$ in (2.111) as the parameter $i \hbar$, where $\hbar \rightarrow 0$ is equivalent to the classical limit of the commutator relations. Since in a quantisation procedure, we go from $\left\{\bar{L}_{n}, \bar{L}_{m}\right\}_{P . B .} \rightarrow \frac{1}{i \hbar}\left[\hat{\bar{L}}_{n}, \hat{\bar{L}}_{m}\right]$, going in reverse would give us the classical Poisson bracket relation

$$
\left\{\bar{L}_{n}, \bar{L}_{m}\right\}_{P . B .}=(n-m) \bar{L}_{n+m}-\frac{24}{12}(k+3)\left(k^{\prime}+3\right)\left(n^{3}-n\right) \delta_{n,-m},
$$

where $\bar{T}_{\text {classical }}(z)=\sum_{n} \bar{L}_{n} z^{-n-2}$. Since the Poisson bracket must be well-defined as $k \rightarrow-3$ and $k^{\prime} \rightarrow \infty$, it will mean that $(k+3)\left(k^{\prime}+3\right)$ must be equal to a finite constant $q$ in these limits. For different values of $q$, the Poisson algebra generated by the Laurent modes of $\bar{T}_{\text {classical }}(z)$, will be a classical Virasoro algebra with different central charge. Note at this point that an equivalence - at the level of the holomorphic chiral algebra - between the $\psi^{\bar{j}_{-}}$ independent sector of the twisted sigma-model on $S L(3) / B$ and the $B$-gauged WZW model on $S L(3)$, will mean that the Laurent modes of $\bar{T}_{\text {classical }}(z)$ and $S(z)$ ought to generate an isomorphic classical algebra. This in turn means that the $\bar{L}_{m}$ 's must generate the same classical Virasoro algebra $\operatorname{Sym}^{\prime}\left(v_{i r}-24\right)$ with central charge -24 that is generated by the Laurent modes $S_{m}$ of the purely bosonic sector of the sigma-model on $S L(3) / B$. Thus, we must have $q=1$, or rather $(k+3)=1 /\left(k^{\prime}+3\right)$.

What is the classical algebra generated by the Laurent modes of $\bar{T}_{\text {classical }}^{(3)}(z)$ ? To ascertain this, first recall that it was argued that the Laurent modes of $T_{\text {total }}^{(3)}(z)$ and $T_{\text {total }}(z)$ will together generate a quantum $\mathcal{W}_{3}$-algebra of central charge $c=50-24\left(k^{\prime}+3\right)-24 /\left(k^{\prime}+3\right)$. 
Hence, the Laurent modes $\hat{L}_{n}^{(3)}$ of $T_{\text {total }}^{(3)}(z)=\sum_{n} \hat{L}_{n}^{(3)} z^{-n-3}$ and the $\hat{L}_{n}$ 's will obey the following quantum commutator relations

$$
\left[\hat{L}_{n}, \hat{L}_{m}^{(3)}\right]=(2 n-m) \hat{L}_{n+m}^{(3)}
$$

and

$$
\begin{aligned}
{\left[\hat{L}_{m}^{(3)}, \hat{L}_{n}^{(3)}\right] } & =\frac{50-24\left(k^{\prime}+3\right)-24 /\left(k^{\prime}+3\right)}{360} m\left(m^{2}-1\right)\left(m^{2}-4\right) \delta_{m,-n} \\
& +(m-n)\left(\frac{1}{15}(m+n+3)(m+n+2)-\frac{1}{6}(m+2)(n+2)\right) \hat{\bar{L}}_{m+n} \\
& +\left(\frac{16}{22+5\left[50-24\left(k^{\prime}+3\right)-24 /\left(k^{\prime}+3\right)\right]}\right)(m-n)\left(\sum_{p} \hat{\bar{L}}_{m+n-p} \hat{\bar{L}}_{p}\right) \\
& -\left(\frac{16}{22+5\left[50-24\left(k^{\prime}+3\right)-24 /\left(k^{\prime}+3\right)\right]}\right)(m-n)\left(\frac{3}{10}(m+n+3)(m+n+2) \hat{\bar{L}}_{m+n}\right) .
\end{aligned}
$$

Therefore, from (2.113) and (2.114), the commutator relations involving the $\hat{\bar{L}}_{n}$ modes of $\bar{T}_{\text {total }}(z)=\sum_{n} \hat{\bar{L}}_{n} z^{-n-2}$ and the $\hat{\bar{L}}_{n}^{(3)}$ modes of $\bar{T}_{\text {total }}^{(3)}(z)=\sum_{n} \hat{\bar{L}}_{n}^{(3)} z^{-n-2}$, will be given by

$$
\left[\hat{\bar{L}}_{n}, \hat{\bar{L}}_{m}^{(3)}\right]=(k+3)(2 n-m) \hat{\bar{L}}_{n+m}^{(3)}
$$

and

$$
\begin{aligned}
& {\left[\hat{\bar{L}}_{m}^{(3)}, \hat{\bar{L}}_{n}^{(3)}\right]=} \\
& (k+3)\left[\frac{50(k+3)-24(k+3)\left(k^{\prime}+3\right)-\frac{24(k+3)}{\left(k^{\prime}+3\right)}}{360} m\left(m^{2}-1\right)\left(m^{2}-4\right) \delta_{m,-n}\right] \\
& +(k+3)\left[(m-n)\left(\frac{1}{15}(m+n+3)(m+n+2)-\frac{1}{6}(m+2)(n+2)\right) \hat{\bar{L}}_{m+n}\right] \\
& +(k+3)\left[\left(\frac{16}{22(k+3)+5\left[50(k+3)-24(k+3)\left(k^{\prime}+3\right)-\frac{24(k+3)}{\left(k^{\prime}+3\right)}\right]}\right)(m-n)\left(\sum_{p} \hat{\bar{L}}_{m+n-p} \hat{\bar{L}}_{p}\right)\right] \\
& -(k+3)\left[\left(\frac{16}{22+5\left[50-24\left(k^{\prime}+3\right)-24 /\left(k^{\prime}+3\right)\right]}\right)(m-n)\left(\frac{3}{10}(m+n+3)(m+n+2) \hat{\bar{L}}_{m+n}\right)\right] \text {. }
\end{aligned}
$$


At $k=-3, \bar{T}_{\text {total }}^{(3)}(z)$ will cease to have a quantum definition, and it will reduce to its classical counterpart $\bar{T}_{\text {classical }}^{(3)}(z)$. Hence, the $k \rightarrow-3$ (and $k^{\prime} \rightarrow \infty$ ) limit of the commutator relations in (2.115) and (2.116), can be interpreted as their classical limits. Therefore, one can view the term $(k+3)$ in (2.115) and (2.116) as the parameter $i \hbar$, where $\hbar \rightarrow 0$ is equivalent to the classical limit of the commutator relations. Since in a quantisation procedure, we go from $\left\{\bar{D}_{n}, \bar{D}_{m}\right\}_{P . B .} \rightarrow \frac{1}{i \hbar}\left[\hat{\bar{D}}_{n}, \hat{\bar{D}}_{m}\right]$ for any classical observable $\bar{D}_{n}$, going in reverse would give us the classical Poisson bracket relations

$$
\left\{\bar{L}_{n}, \bar{L}_{m}^{(3)}\right\}_{P . B .}=(2 n-m) \bar{L}_{n+m}^{(3)}
$$

and

$$
\begin{aligned}
\left\{\bar{L}_{m}^{(3)}, \bar{L}_{n}^{(3)}\right\}_{P . B .}= & {\left[\frac{-24(k+3)\left(k^{\prime}+3\right)}{360} m\left(m^{2}-1\right)\left(m^{2}-4\right) \delta_{m,-n}\right] } \\
& +\left[(m-n)\left(\frac{1}{15}(m+n+3)(m+n+2)-\frac{1}{6}(m+2)(n+2)\right) \bar{L}_{m+n}\right] \\
& +\left[\left(\frac{-4}{30(k+3)\left(k^{\prime}+3\right)}\right)(m-n)\left(\sum_{p} \bar{L}_{m+n-p} \bar{L}_{p}\right)\right],
\end{aligned}
$$

where $\bar{T}_{\text {classical }}^{(3)}(z)=\sum_{n} \bar{L}_{n}^{(3)} z^{-n-3}$. Since the Poisson bracket in (2.118) must be well-defined as $k \rightarrow-3$ and $k^{\prime} \rightarrow \infty$, it will mean that $(k+3)\left(k^{\prime}+3\right)$ must be equal to a finite constant $q$ in these limits. For different values of $q$, the total Poisson algebra generated by the Laurent modes of $\bar{T}_{\text {classical }}(z)$ and $\bar{T}_{\text {classical }}^{(3)}(z)$ in (2.112), (2.117) and (2.118), will be a classical $\mathcal{W}_{3}$-algebra with different central charges. Note at this point that an equivalence - at the level of the holomorphic chiral algebra - between the $\psi^{\bar{j}}$-independent sector of the twisted sigma-model on $S L(3) / B$ and the $B$-gauged WZW model on $S L(3)$, will mean that the Laurent modes of $\left(\bar{T}_{\text {classical }}(z), \bar{T}_{\text {classical }}^{(3)}(z)\right)$ and $\left(S(z), S^{(3)}(z)\right)$ ought to generate an isomorphic classical algebra. This means that we must have $q=1$, or rather $(k+3)=$ $1 /\left(k^{\prime}+3\right)$, so that the total Poisson algebra will be given by

$$
\begin{gathered}
\left\{\bar{L}_{n}, \bar{L}_{m}\right\}_{P . B .}=(n-m) \bar{L}_{n+m}-\frac{24}{12}\left(n^{3}-n\right) \delta_{n,-m}, \\
\left\{\bar{L}_{n}, \bar{L}_{m}^{(3)}\right\}_{P . B .}=(2 n-m) \bar{L}_{n+m}^{(3)}
\end{gathered}
$$


and

$$
\begin{aligned}
\left\{\bar{L}_{m}^{(3)}, \bar{L}_{n}^{(3)}\right\}_{P . B .}= & -\frac{24}{360} m\left(m^{2}-1\right)\left(m^{2}-4\right) \delta_{m,-n} \\
& +(m-n)\left(\frac{1}{15}(m+n+3)(m+n+2)-\frac{1}{6}(m+2)(n+2)\right) \bar{L}_{m+n} \\
& -\frac{4}{30}(m-n)\left(\sum_{p} \bar{L}_{m+n-p} \bar{L}_{p}\right)
\end{aligned}
$$

the classical $\mathcal{W}_{3}$-algebra with central charge -24 generated by the Laurent modes $S_{m}$ and $S_{m}^{(3)}$ in in (2.91), (2.89) and (2.90). Note also that this algebra coincides with $\mathcal{W}_{\infty}\left(\widehat{\mathfrak{s l}}_{3}\right)$, the classical $\mathcal{W}$-algebra associated to $\widehat{\mathfrak{s l}}_{3}$ at level $k^{\prime} \rightarrow \infty$ obtained via a DS reduction scheme [7]. Since the $S_{m}$ 's and $S_{m}^{(3)}$ 's which correspond respectively to the $\bar{L}_{n}$ 's and $\bar{L}_{m}^{(3)}$ 's span $\mathfrak{z}\left(\widehat{\mathfrak{s l}}_{3}\right)$, and since for $\mathfrak{g}=\mathfrak{s l}_{3}={ }^{L} \mathfrak{g}$, we have $h^{\vee}={ }^{L} h^{\vee}=3$, and $r^{\vee}=1$, where $r^{\vee}$ is the lacing number of $\mathfrak{g}$, we see that an equivalence - at the level of the holomorphic chiral algebra - between the $\psi^{\bar{j}}$-independent sector of the twisted sigma-model on $S L(3) / B$ and the $B$-gauged WZW model on $S L(3)$, will imply an isomorphism of Poisson algebras

$$
\mathfrak{z}(\widehat{\mathfrak{g}}) \cong \mathcal{W}_{\infty}\left({ }^{L} \widehat{\mathfrak{g}}\right)
$$

and the level relation

$$
\left(k+h^{\vee}\right) r^{\vee}=\frac{1}{\left(k^{\prime}+{ }^{L} h^{\vee}\right)} .
$$

Note at this point that the purely bosonic, $\psi^{\bar{j}}$-independent sector of the twisted sigmamodel on $S L(3) / B$, can be described, via (2.31), by a bosonic string on $S L(3) / B$. On the other hand, note that since a bosonic string on a group manifold $G$ can be described as a WZW model on $G$, it will mean that the $B$-gauged WZW model on $S L(3)$ can be interpreted as a $B$-gauged bosonic string on $S L(3)$. Thus, we see that an equivalence, at the level of the holomorphic chiral algebra, between a bosonic string on $S L(3) / B$ and a $B$-gauged version of itself on $S L(3)$ - a statement which stems from the ubiquitous notion that one can always physically interpret a geometrical symmetry of the target space as a gauge symmetry in the worldsheet theory - will imply an isomorphism of classical $\mathcal{W}$-algebras and a level relation which underlie a geometric Langlands correspondence for $G=S L(3)$ ! Notice also that the correspondence between the $k \rightarrow-3$ and $k^{\prime} \rightarrow \infty$ limits (within the context of the above Poisson algebras) is indeed consistent with the relation (2.123). These 
limits define a "classical" geometric Langlands correspondence. A "quantum" generalisation of the correspondence for $S L(3)$ can be defined for other values of $k$ and $k^{\prime}$ that satisfy the relation (2.123), but with the isomorphism of (2.122) replaced by an isomorphism of quantum $\mathcal{W}$-algebras (derived from a DS-reduction scheme) associated to $\widehat{\mathfrak{s l}}_{3}$ at levels $k$ and $k^{\prime}$ respectively [6].

\section{An Equivalence of Classical Holomorphic Chiral Algebras and the Geometric Langlands Correspondence for $G=S L(N)$}

We shall now proceed to show that our observations in $\S 2$ for $G=S L(2)$ and $S L(3)$ can be extended to any $G=S L(N)$.

To this end, we shall first discuss the twisted sigma-model on $S L(N) / B$, and elaborate on the higher-order Casimir invariant operators which generalise the Segal-Sugawara tensor $S(z)$ to higher-spin analogs of itself that we will denote as $S^{(3)}(z), S^{(4)}(z), \ldots, S^{(N)}(z)$. We shall show that $S(z), S^{(3)}(z), S^{(4)}(z), \ldots, S^{(N)}(z)$ have Laurent modes which generate

the centre $\mathfrak{z}\left(\widehat{\mathfrak{s l}}_{N}\right)$ of the completed universal enveloping algebra of $\widehat{\mathfrak{s l}}_{N}$ at the critical level $k=-h^{\vee}$, where a module of $\widehat{\mathfrak{s l}}_{N}$ at $k=-h^{\vee}$ is always furnished by the global sections of the sheaf of CDO's on $S L(N) / B$ corresponding to local operators in the quantum holomorphic chiral algebra of the twisted sigma-model on $S L(N) / B$. We shall also show that $S(z), S^{(3)}(z), S^{(4)}(z), \ldots, S^{(N)}(z)$ exist only in the classical holomorphic chiral algebra of the purely bosonic sector of the twisted sigma-model on $S L(N) / B$, and that moreover, their Laurent modes which span $\mathfrak{z}\left(\widehat{\mathfrak{s l}}_{N}\right)$ - an ingredient which furnishes the left-hand side of the $\mathcal{W}$-algebra duality - will generate a classical $\mathcal{W}_{N}$-algebra.

Next, we shall show that the holomorphic BRST-cohomology of the B-gauged WZW model on $S L(N)$ at level $k^{\prime}$ introduced in $\S 2.2$ actually furnishes a physical realisation of the algebraic DS reduction scheme that defines the set of fields with spins $2,3, \ldots N$ whose Laurent modes generate $\mathcal{W}_{k^{\prime}}\left(\widehat{\mathfrak{s l}}_{N}\right)$, the $\mathcal{W}$-algebra associated to some affine Lie algebra $\widehat{\mathfrak{s l}}_{N}$ at level $k^{\prime}$. Consequently, the classical, holomorphic BRST-cohomology of the $B$-gauged WZW model on $S L(N)$ will reproduce the holomorphic classical fields with spins $2,3, \ldots, N$ that have Laurent modes which generate the Poisson algebra $\mathcal{W}_{\infty}\left(\widehat{\mathfrak{s l}}_{N}\right)$, the classical $\mathcal{W}$-algebra from the DS-reduction scheme in the limit of $k^{\prime} \rightarrow \infty$ - an ingredient which furnishes the right-hand side of the $\mathcal{W}$-algebra duality.

Finally, we shall show that that an equivalence, at the level of the holomorphic chiral algebra, between a bosonic string on $S L(N) / B$ and a $B$-gauged version of itself on $S L(N)$ - 
a statement which stems from the ubiquitous notion that one can always physically interpret a geometrical symmetry of the target space as a gauge symmetry in the worldsheet theory - will imply an isomorphism of classical $\mathcal{W}$-algebras and a level relation which underlie a geometric Langlands correspondence for $G=S L(N)$.

\subsection{The Twisted Sigma-Model on $S L(N) / B$ and its Classical Holomorphic Chiral Algebra}

Now, let us take $X=S L(N) / B$, where $B$ is the subgroup of upper triangular matrices of $S L(3)$ with a nilpotent Lie algebra $\mathfrak{b}$. Note that one can cover $X$ with $N$ ! open charts $U_{w}$ where $w=1,2, \ldots, N$ !, such that each open chart $U_{w}$ can be identified with the affine space $\mathbb{C}^{N(N-1) / 2}$. Hence, the sheaf of CDO's in any $U_{w}$ can be described by $N(N-1) / 2$ free $\beta \gamma$ systems with the action

$$
I=\sum_{i=1}^{N(N-1) / 2} \frac{1}{2 \pi} \int\left|d^{2} z\right| \beta_{i} \partial_{\bar{z}} \gamma^{i}
$$

As before, the $\beta_{i}$ 's and $\gamma^{i}$ 's are fields of dimension $(1,0)$ and $(0,0)$ respectively. They obey the standard free-field OPE's; there are no singularities in the operator products $\beta_{i}(z) \cdot \beta_{i}\left(z^{\prime}\right)$ and $\gamma^{i}(z) \cdot \gamma^{i}\left(z^{\prime}\right)$, while

$$
\beta_{i}(z) \gamma^{j}\left(z^{\prime}\right) \sim-\frac{\delta_{i}^{j}}{z-z^{\prime}}
$$

Similarly, the sheaf of CDO's in a neighbouring intersecting chart $U_{w+1}$ is described by $N(N-1) / 2$ free $\tilde{\beta} \tilde{\gamma}$ systems with action

$$
I=\sum_{i=1}^{N(N-1) / 2} \frac{1}{2 \pi} \int\left|d^{2} z\right| \tilde{\beta}_{i} \partial_{\bar{z}} \tilde{\gamma}^{i}
$$

where the $\tilde{\beta}_{i}$ and $\tilde{\gamma}^{i}$ fields obey the same OPE's as the $\beta_{i}$ and $\gamma^{i}$ fields. In other words, the non-trivial OPE's are given by

$$
\tilde{\beta}_{i}(z) \tilde{\gamma}^{j}\left(z^{\prime}\right) \sim-\frac{\delta_{i}^{j}}{z-z^{\prime}}
$$

In order to describe a globally-defined sheaf of CDO's, one will need to glue the free conformal field theories with actions (3.1) and (3.3) in the overlap region $U_{w} \cap U_{w+1}$ for every $w=1,2, \ldots N$ !, where $U_{1+N !}=U_{1}$. To do so, one must use the admissible automorphisms of the free conformal field theories defined in (A.29)-(A.30) to glue the free-fields together. In the case of $X=S L(N) / B$, the relation between the coordinates in $U_{w}$ and $U_{w+1}$ will mean 
that the $\tilde{\gamma}^{i}$ 's in $U_{w+1}$ will be related to the $\gamma^{i}$ 's in $U_{w}$ via the relation $[\tilde{\gamma}]=\left[V_{w+1}\right]^{-1}\left[V_{w}\right][\gamma]$, where the $[N(N-1) / 2] \times[N(N-1) / 2]$ matrices $\left[V_{w+1}\right]$ and $\left[V_{w}\right]$ are realisations of the $S_{N}$ permutation subgroup of $G L(N)$ associated to the open charts $U_{w+1}$ and $U_{w}$ respectively, and $[\gamma]$ is a $[N(N-1) / 2] \times 1$ column matrix with the $\gamma^{i}$ 's as entries. By substituting this relation between the $\tilde{\gamma}^{i}$ 's and $\gamma^{i}$ 's in (A.29)-(A.30), one will have the admissible automorphisms of the fields, which can then be used to glue together the local sheaves of CDO's in the overlap region $U_{w} \cap U_{w+1}$ for every $w=1,2, \ldots, N$ !. These gluing relations for the free fields can be written as

$$
\begin{aligned}
& \tilde{\gamma}^{i}=\left[V_{w+1}^{-1} \cdot V_{w}\right]^{i}{ }_{j} \gamma^{j}, \\
& \tilde{\beta}_{i}=\beta_{k} D^{k}{ }_{i}+\partial_{z} \gamma^{j} E_{i j},
\end{aligned}
$$

where $i, j, k=1,2, \ldots, N(N-1) / 2$. Here, $D$ and $E$ are $[N(N-1) / 2] \times[N(N-1) / 2]$ matrices, whereby $\left[\left(D^{T}\right)^{-1}\right]_{i}{ }^{k}=\partial_{i}\left[V_{w+1}^{-1} \cdot V_{w}\right]_{j}{ }_{j} \gamma^{j}$ and $[E]_{i j}=\partial_{i} B_{j}$. It can be verified that $\tilde{\beta}$ and $\tilde{\gamma}$ obey the correct OPE's amongst themselves. Moreover, let $R_{w}$ represent a transformation of the fields in going from $U_{w}$ to $U_{w+1}$. One can indeed verify that there is no anomaly to a global definition of a sheaf of CDO's on $X=S L(N) / B$ - a careful computation will reveal that one will get the desired composition maps $\left(R_{N !} \ldots R_{4} R_{3} R_{2} R_{1}\right) \cdot \gamma^{j}=\gamma^{j}$ and $\left(R_{N !} \ldots R_{4} R_{3} R_{2} R_{1}\right) \cdot \beta_{i}=\beta_{i}$. Again, this is just a statement that one can always define a sheaf of CDO's on any flag manifold $S L(N) / B$ [11].

Global Sections of the Sheaf of CDO's on $X=S L(N) / B$

Since $X=S L(N) / B$ is of complex dimension $N(N-1) / 2$, the chiral algebra $\mathcal{A}$ will be given by $\mathcal{A}=\bigoplus_{g_{R}=0}^{g_{R}=N(N-1) / 2} H^{g_{R}}\left(X, \widehat{\mathcal{O}}_{X}^{c h}\right)$ as a vector space. As before, it would suffice for our purpose to concentrate on just the purely bosonic sector of $\mathcal{A}$ - from our $\bar{Q}_{+}$-Cech cohomology dictionary, this again translates to studying only the global sections in $H^{0}\left(X, \widehat{\mathcal{O}}_{X}^{c h}\right)$.

According to theorem 5.13 of [11], one can always find elements in $H^{0}\left(M, \widehat{\mathcal{O}}_{M}^{c h}\right)$ for any flag manifold $M=S L(N) / B$, that will furnish a module of an affine $S L(N)$ algebra at the critical level. This means that one can always find dimension one global sections of the sheaf $\widehat{\mathcal{O}}_{X}^{c h}$ that correspond to $\psi^{\bar{i}}$-independent currents $J^{a}(z)$ for $a=1,2, \ldots \operatorname{dim} \mathfrak{s l}_{N}$, that satisfy the OPE's of an affine $S L(N)$ algebra at the critical level $k=-h^{\vee}$ :

$$
J_{a}(z) J_{b}\left(z^{\prime}\right) \sim-\frac{h^{\vee} d_{a b}}{\left(z-z^{\prime}\right)^{2}}+\sum_{c} f_{a b}{ }^{c} \frac{J_{c}\left(z^{\prime}\right)}{\left(z-z^{\prime}\right)}
$$

where $h^{\vee}$ is the dual Coxeter number of the Lie algebra $\mathfrak{s l}_{N}$, and $d_{a b}$ is its Cartan-Killing 
metric 10 Since these are global sections, it will be true that $\widetilde{J}_{a}(z)=J_{a}(z)$ on any $U_{w} \cap U_{w+1}$ for all $a$. Moreover, from our $\bar{Q}_{+}$-Cech cohomology dictionary, they will be $\bar{Q}_{+}$-closed chiral vertex operators that are holomorphic in $z$, which means that one can expand them in a Laurent series that allows an affinisation of the $S L(N)$ algebra generated by their resulting zero modes. The space of these operators obeys all the physical axioms of a chiral algebra except for reparameterisation invariance on the $z$-plane or worldsheet $\Sigma$. We will substantiate this last statement next by showing that the holomorphic stress tensor fails to exist in the $\bar{Q}_{+}$-cohomology at the quantum level. Again, this observation will be important in our discussion of a geometric Langlands correspondence for $G=S L(N)$.

\section{The Segal-Sugawara Tensor and the Classical Holomorphic Chiral Algebra}

Recall that for any affine algebra $\widehat{\mathfrak{s l}}_{N}$ at level $k \neq-h^{\vee}$, one can construct the corresponding stress tensor out of the currents of $\widehat{\mathfrak{s l}}_{N}$ via a Segal-Sugawara construction [16]:

$$
T(z)=\frac{: d^{a b} J_{a} J_{b}(z):}{k+h^{\vee}},
$$

As required, for every $k \neq-h^{\vee}$, the modes of the Laurent expansion of $T(z)$ will span a Virasoro algebra. In particular, $T(z)$ will generate holomorphic reparametrisations of the coordinates on the worldsheet $\Sigma$. Notice that this definition of $T(z)$ in (3.8) is ill-defined when $k=-h^{\vee}$. Nevertheless, one can always associate $T(z)$ with the Segal-Sugawara operator $S(z)$ that is well-defined at any finite level, whereby

$$
S(z)=\left(k+h^{\vee}\right) T(z)
$$

and

$$
S(z)=: d^{a b} J_{a} J_{b}(z):
$$

From (3.9), we see that $S(z)$ generates, in its OPE's with other field operators, $\left(k+h^{\vee}\right)$ times the transformations usually generated by the stress tensor $T(z)$. Therefore, at the

\footnotetext{
${ }^{10}$ Note that one can consistently introduce appropriate fluxes to deform the level away from $-h^{\vee}$ - recall from our discussion in $\S$ A.7 that the $E_{i j}=\partial_{i} B_{j}$ term in (3.6) is related to the fluxes that correspond to the moduli of the chiral algebra, and since this term will determine the level $k$ of the affine $S L(N)$ algebra via the identification of the global sections $\tilde{\beta}_{i}$ with the affine currents valued in the subalgebra of $\mathfrak{s l}_{N}$ associated to its positive roots, turning on the relevant fluxes will deform $k$ away from $-h^{\vee}$. Henceforth, whenever we consider $k \neq-h^{\vee}$, we really mean turning on fluxes in this manner. This point of departure will be important in a forthcoming paper where we aim to investigate the physical interpretation of a "quantum" geometric Langlands correspondence in the context of CDO's.
} 
level $k=-h^{\vee}, S(z)$ generates no transformations at all - its OPE's with all other field operators are trivial. This is equivalent to saying that the holomorphic stress tensor does not exist at the quantum level, since $S(z)$, which is the only well-defined operator at this level that could possibly generate the transformation of fields under an arbitrary holomorphic reparametrisation of the worldsheet coordinates on $\Sigma$, acts by zero in the OPE's.

Despite the fact that $S(z)$ will cease to exist in the spectrum of physical operators associated to the twisted sigma-model on $X=S L(N) / B$ at the quantum level, it will nevertheless exist as a field in its classical $\bar{Q}_{+}$-cohomology or holomorphic chiral algebra. One can convince oneself that this is true as follows. Firstly, from our $\bar{Q}_{+}$-Cech cohomology dictionary, since the $J_{a}(z)$ 's are in $H^{0}\left(X, \widehat{\mathcal{O}}_{X}^{c h}\right)$, it will mean that they are in the $\bar{Q}_{+}$-cohomology of the sigma-model at the quantum level. Secondly, since quantum corrections can only annihilate cohomology classes and not create them, it will mean that the $J_{a}(z)$ 's will be in the classical $\bar{Q}_{+}$-cohomology of the sigma-model, i.e., the currents are $\bar{Q}_{+}$-closed and are therefore invariant under the transformations generated by $\bar{Q}_{+}$in the absence of quantum corrections. Hence, one can readily see that $S(z)$ in $(3.10)$ will also be $\bar{Q}_{+}$-closed at the classical level. Lastly, recall from section 2.3 that $\left[\bar{Q}_{+}, T(z)\right]=0$ such that $T(z) \neq\left\{\bar{Q}_{+}, \cdots\right\}$ in the absence of quantum corrections to the action of $\bar{Q}_{+}$in the classical theory. Note also that the integer $h^{\vee}$ in the factor $\left(k+h^{\vee}\right)$ of the expression $S(z)$ in (3.9), is due to a shift by $h^{\vee}$ in the level $k$ because of quantum renormalisation effects [17], i.e., the classical expression of $S(z)$ for a general level $k$ can actually be written as $S(z)=k T(z)$, and therefore, one will have $\left[\bar{Q}_{+},-h^{\vee} T(z)\right]=\left[\bar{Q}_{+}, S(z)\right]=0$, where $S(z) \neq\left\{\bar{Q}_{+}, \cdots\right\}$ in the classical theory. Therefore, $S(z)$ will be a spin-two field in the classical holomorphic chiral algebra of the purely bosonic sector of the twisted sigma-model on $X=S L(N) / B$. This observation is also consistent with the fact that $S(z)$ fails to correspond to a global section of the sheaf $\mathcal{O}_{X}^{c h}$ of CDO's - note that in our case, we actually have $S(z)=-h^{\vee} T(z)$ in the classical theory, and this will mean that under quantum corrections to the action of $\bar{Q}_{+}$, we will have $\left[\bar{Q}_{+}, S_{z z}\right]=-h^{\vee} \partial_{z}\left(R_{i \bar{j}} \partial_{z} \phi^{i} \psi^{\bar{j}}\right) \neq 0$ ( since $R_{i \bar{j}} \neq 0$ for any flag manifold $S L(N) / B$ ), which corresponds in the Cech cohomology picture to the expression $\widetilde{\widehat{S}}(z)-\widehat{S}(z) \neq 0$, i.e., $\widehat{S}(z)$, the Cech cohomology counterpart to the $S(z)$ operator, will fail to be in $H^{0}\left(X, \widehat{\mathcal{O}}_{X}^{c h}\right)$. Consequently, one can always represent $S(z)$ by a classical $c$-number. This point will be important when we discuss how one can define Hecke eigensheaves that will correspond to flat ${ }^{L} G$-bundles on a Riemann surface $\Sigma$ in our physical interpretation of the geometric Langlands correspondence for $G=S L(N)$.

The fact that $S(z)$ acts trivially in any OPE with other field operators implies that its 
Laurent modes will commute with the Laurent modes of any of these other field operators; in particular, they will commute with the Laurent modes of the $J_{a}(z)$ currents - in other words, the Laurent modes of $S(z)$ will span the centre $\mathfrak{z}\left(\widehat{\mathfrak{s l}}_{N}\right)$ of the completed universal enveloping algebra of the affine $S L(N)$ algebra $\widehat{\mathfrak{s l}}_{N}$ at the critical level $k=-h^{\vee}$ (generated by the Laurent modes of the $J_{a}(z)$ currents in the quantum chiral algebra of the twisted sigma-model on $S L(N) / B$ ). Notice also that $S(z)$ is $\psi^{\bar{j}}$-independent and is therefore purely bosonic in nature. In other words, the local field $S(z)$ exists only in the classical holomorphic chiral algebra of the purely bosonic (or $\psi^{\bar{j}}$-independent) sector of the twisted sigma-model on $X=S L(N) / B$.

\section{A Classical Virasoro Algebra}

Note that since $S(z)$ is holomorphic in $z$ and is of conformal dimension two, one can expand it in terms of a Laurent expansion as

$$
S(z)=\sum_{n \in \mathbb{Z}} \hat{S}_{n} z^{-n-2}
$$

Recall that for the general case of $k \neq-h^{\vee}$, a quantum definition of $S(z)$ exists, such that

the $\hat{S}_{n}$ modes of the Laurent expansion can be related to the $J_{a, n}$ modes of the $\widehat{\mathfrak{s l}}_{N}$ currents through the quantum commutator relations

$$
\begin{aligned}
{\left[\hat{S}_{n}, J_{a, m}\right] } & =-\left(k+h^{\vee}\right) m J_{a, n+m}, \\
{\left[\hat{S}_{n}, \hat{S}_{m}\right] } & =\left(k+h^{\vee}\right)\left((n-m) \hat{S}_{n+m}+\frac{k \operatorname{dim} \mathfrak{s l}_{N}}{12}\left(n^{3}-n\right) \delta_{n,-m}\right),
\end{aligned}
$$

where $a=1,2, \ldots, \operatorname{dim} \mathfrak{s l}_{N}$. If we now let $k=-h^{\vee}$, we will have $\left[\hat{S}_{n}, J_{a, m}\right]=\left[\hat{S}_{n}, \hat{S}_{m}\right]=0$ the $S_{m}$ 's thus generate the (classical) centre of the completed universal enveloping algebra of $\widehat{\mathfrak{s l}}_{N}$ as mentioned above.

Since we now understand that $S(z)$ must be a holomorphic classical field at $k=-h^{\vee}$, let us rewrite the Laurent expansion of $S(z)$ as

$$
S(z)=\sum_{n \in \mathbb{Z}} S_{n} z^{-n-2}
$$

so as to differentiate the classical modes of expansion $S_{n}$ from their quantum counterpart $\hat{S}_{n}$ in (3.9). Unlike the $\hat{S}_{n}$ 's which obey the quantum commutator relations in (3.13) for an arbitrary level $k \neq-h^{\vee}$, the $S_{n}$ 's, being the modes of a Laurent expansion of a classical 
field, will instead obey Poisson bracket relations that define a certain classical algebra when $k=-h^{\vee}$.

Based on our arguments thus far, we learn that the quantum version of $S(z)$ as expressed in (3.9), must reduce to its classical counterpart as expressed in (3.14), when $k=-h^{\vee}$. In other words, one can see that by taking $\left(k+h^{\vee}\right) \rightarrow 0$, we are going to the classical limit. This is analogous to taking the $\hbar \rightarrow 0$ limit in any quantum mechanical theory whenever one wants to ascertain its classical counterpart. In fact, by identifying $\left(k+h^{\vee}\right)$ with $i \hbar$, and by noting that one must make the replacement from Possion brackets to commutators via $\left\{S_{n}, S_{m}\right\}_{P . B .} \rightarrow \frac{1}{i \hbar}\left[\hat{S}_{n}, \hat{S}_{m}\right]$ in quantising the $S_{n}$ 's into operators, we can ascertain the classical algebra generated by the $S_{n}$ 's from $(3.13)$ as

$$
\left\{S_{n}, S_{m}\right\}_{P . B .}=(n-m) S_{n+m}-\frac{h^{\vee}\left(\operatorname{dimsl}_{N}\right)}{12}\left(n^{3}-n\right) \delta_{n,-m}
$$

Since we have the classical relation $S(z) \sim T(z)$, it means that we can interpret the $S_{n}$ modes as the Virasoro modes of the Laurent expansion of the classical stress tensor field $T(z)$. In other words, the $S_{n}$ 's generate a classical Virasoro algebra with central charge $-h^{\vee}\left(\operatorname{dims} \mathfrak{s}_{N}\right)$ as given by (3.15). This is can be denoted mathematically as the Virasoro Poisson algebra

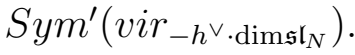

Higher-Spin Analogs of the Segal-Sugawara Tensor and the Classical Holomorphic Chiral Algebra

For an affine $S L(N)$ algebra where $N>2$, one can generalise the Sugawara formalism to construct higher-spin analogs of the holomorphic stress tensor with the currents. These higher-spin analogs are called Casimir operators, and were first constructed in [25].

In the context of an affine $S L(N)$ algebra with a module that is furnished by the global sections of the sheaf of CDO's on $X=S L(N) / B$, a spin- $s_{i}$ analog of the holomorphic stress tensor will be given by the $s_{i}{ }^{\text {th }}$-order Casimir operator 12

$$
T^{\left(s_{i}\right)}(z)=\frac{: \tilde{d}^{a_{1} a_{2} a_{3} \ldots a_{s_{i}}}(k)\left(J_{a_{1}} J_{a_{2}} \ldots J_{a_{s_{i}}}\right)(z):}{k+h^{\vee}},
$$

where $\tilde{d}^{a_{1} a_{2} a_{3} \ldots a_{s_{i}}}(k)$ is a completely symmetric traceless $\mathfrak{s l}_{N}$-invariant tensor of rank $s_{i}$ that depends on the level $k$ of the affine $S L(N)$ algebra. It is also well-defined and finite at $k=$ $-h^{\vee}$. The superscript on $T^{\left(s_{i}\right)}(z)$ just denotes that it is a spin- $s_{i}$ analog of $T(z)$. Note that $i=1,2, \ldots, \operatorname{rank}\left(\mathfrak{s l}_{N}\right)$, and the spins $s_{i}$ can take the values $1+e_{i}$, where $e_{i}=1,2, \ldots, N-1$. 
Thus, one can have $\operatorname{rank}\left(\mathfrak{s l}_{N}\right)$ of these Casimir operators, and the spin-2 Casimir operator is just the holomorphic stress tensor from the usual Sugawara construction.

As with $T(z)$ in (3.8), $T^{\left(s_{i}\right)}(z)$ is ill-defined when $k=-h^{\vee}$. Nevertheless, one can always make reference to a spin- $s_{i}$ analog of the Segal-Sugawara tensor $S^{\left(s_{i}\right)}(z)$ that is well-defined for any finite value of $k$, where its relation to $T^{\left(s_{i}\right)}(z)$ is given by

$$
S^{\left(s_{i}\right)}(z)=\left(k+h^{\vee}\right) T^{\left(s_{i}\right)}(z)
$$

and

$$
S^{\left(s_{i}\right)}(z)=: \tilde{d}^{a_{1} a_{2} a_{3} \ldots a_{s_{i}}}(k)\left(J_{a_{1}} J_{a_{2}} \ldots J_{a_{s_{i}}}\right)(z): .
$$

That is, the operator $S^{\left(s_{i}\right)}(z)$ generates in its OPE's with all other operators of the quantum theory, $\left(k+h^{\vee}\right)$ times the field transformations typically generated by $T^{\left(s_{i}\right)}(z)$.

Notice however, that at $k=-h^{\vee}, S^{\left(s_{i}\right)}(z)$ acts by zero in its OPE with any other operator. This is equivalent to saying that $T^{\left(s_{i}\right)}(z)$ does not exist as a quantum operator at all, since the only well-defined operator $S^{\left(s_{i}\right)}(z)$ which is supposed to generate the field transformations associated to $T^{\left(s_{i}\right)}(z)$, act by zero and thus generate no field transformations at all. From our $\bar{Q}_{+}$-Cech cohomology dictionary, this means that the $\psi^{\bar{i}}$-independent operator $T^{\left(s_{i}\right)}(z)$ will fail to correspond to a dimension $s_{i}$ global section of $\widehat{\mathcal{O}}_{X}^{c h}$. Since we have, at the classical level, the relation $S^{\left(s_{i}\right)}(z)=-h^{\vee} T^{\left(s_{i}\right)}(z)$, it will mean that $S^{\left(s_{i}\right)}(z)$ will also fail to correspond to a dimension $s_{i}$ global section of $\widehat{\mathcal{O}}_{X}^{c h}$. Thus, $S^{\left(s_{i}\right)}(z)$ will fail to be an operator at the quantum level. Is it even a spin- $s_{i}$ field in the classical holomorphic chiral algebra of the twisted sigma-model on $S L(N) / B$, one might ask. The answer is yes. To see this, recall that each of the $J_{a_{k}}(z)$ 's are separately $\bar{Q}_{+}$-invariant and not $\bar{Q}_{+}$-exact at the classical level. Therefore, the classical counterpart of $S^{\left(s_{i}\right)}(z)$ in (3.18) must also be such, which in turn means that it will be in the classical $\bar{Q}_{+}$-cohomology and hence classical chiral algebra of the twisted sigma-model on $S L(N) / B$.

The fact that the $S^{\left(s_{i}\right)}(z)$ 's act trivially in any OPE with other field operators implies that their Laurent modes will commute with the Laurent modes of any other operator; in particular, they will commute with the Laurent modes of the currents $J_{a}(z)$ for $a=$ $1,2, \ldots, \operatorname{dims} \mathfrak{s}_{N}$ - in other words, the Laurent modes of all $\operatorname{rank}\left(\mathfrak{s l}_{N}\right)$ of the $S^{\left(s_{i}\right)}(z)$ fields will span fully the centre $\mathfrak{z}\left(\widehat{\mathfrak{s l}}_{N}\right)$ of the completed universal enveloping algebra of $\widehat{\mathfrak{s l}}_{N}$ at the critical level $k=-h^{\vee}$ (which is in turn generated by the Laurent modes of the $J_{a}(z)$ currents of the quantum chiral algebra of the twisted sigma-model on $S L(N) / B$ ). Last but not least, notice that the $S^{\left(s_{i}\right)}(z)$ fields are also $\psi^{\bar{j}}$-independent and are therefore purely bosonic in 
nature. In other words, the local fields $S^{\left(s_{i}\right)}(z)$, for $i=1,2, \ldots \operatorname{rank}\left(\mathfrak{s l}_{N}\right)$, whose Laurent modes will together generate $\mathfrak{z}\left(\widehat{\mathfrak{s l}}_{N}\right)$, exist only in the classical holomorphic chiral algebra of the purely bosonic (or $\psi^{\bar{j}}$-independent) sector of the twisted sigma-model on $X=S L(N) / B$.

\section{A Classical $\mathcal{W}_{N}$-algebra}

For an affine $S L(N)$ algebra at an arbitrary level $k \neq-h^{\vee}$, as in the case of $S(z)$ discussed earlier, a quantum definition of $S^{\left(s_{i}\right)}(z)$ exists. In fact, consider the following operators given by $\bar{S}^{\left(s_{i}\right)}(z)=\eta^{\left(s_{i}\right)}(N): d^{a_{1} a_{2} \ldots a_{s_{i}}}\left(J_{a_{1}} J_{a_{2}} \ldots J_{a_{s_{i}}}\right)(z)$ : for $i=1,2, \ldots, \operatorname{rank}\left(\mathfrak{s l}_{N}\right)$, where $\eta^{\left(s_{i}\right)}(N)$ is just a normalisation that depends on $N$, and $d^{a_{1} a_{2} \ldots a_{s_{i}}}$ is just a rank- $s_{i}$ extension of $d^{a b}$. It can be shown that the $\bar{S}^{\left(s_{i}\right)}(z)$ 's generate a closed Casimir OPE algebra which is isomorphic to a particular $\mathcal{W}_{N}$ OPE algebra [25]. This implies that for $k \neq-h^{\vee}$, every $\bar{S}^{\left(s_{i}\right)}(z)$ and therefore every $S^{\left(s_{i}\right)}(z) \sim: d^{a_{1} a_{2} \ldots a_{s_{i}}}\left(J_{a_{1}} J_{a_{2}} \ldots J_{a_{s_{i}}}\right)(z)$ :, will exist as a quantum operator in some cohomology - the $\bar{Q}_{+}$-cohomology in this instance. This will in turn mean that the set of $S^{\left(s_{i}\right)}(z)=\left(k+h^{\vee}\right) T^{\left(s_{i}\right)}(z)$ operators must also span a closed OPE algebra that is equivalent - at the level of $\bar{Q}_{+}$-cohomology - to this Casimir OPE algebra, when $k \neq-h^{\vee}$. Since we know that for $k \neq-h^{\vee}, T^{(2)}(z)=T(z)$ will generate a Virasoro subalgebra of a closed $\mathcal{W}_{N}$ OPE algebra with central charge $c=k \operatorname{dim}\left(\mathfrak{s l}_{N}\right) /\left(k+h^{\vee}\right)$, it will imply that the $S^{\left(s_{i}\right)}(z)$ 's will satisfy a rescaled (by a factor of $\left(k+h^{\vee}\right)$ ) version of a closed $\mathcal{W}_{N}$ OPE algebra at $c=k \operatorname{dim}\left(\mathfrak{s l}_{N}\right) /\left(k+h^{\vee}\right)$ for $k \neq-h^{\vee}$. Because each $S^{\left(s_{i}\right)}(z)$ is holomorphic in $z$, we can Laurent expand it as

$$
S^{\left(s_{i}\right)}(z)=\sum_{n \in \mathbb{Z}} \hat{S}_{n}^{\left(s_{i}\right)} z^{-n-s_{i}}
$$

At $k \neq-h^{\vee}$, since $S^{(2)}(z)=S(z)$, the Laurent modes $\hat{S}_{n}^{(2)}$ of $S^{(2)}(z)$, will then generate the Virasoro algebra with the following quantum commutator relations given in (3.13):

$$
\left[\hat{S}_{n}^{(2)}, \hat{S}_{m}^{(2)}\right]=\left(k+h^{\vee}\right)\left((n-m) \hat{S}_{n+m}^{(2)}+\frac{k \operatorname{dim} \mathfrak{s l}_{N}}{12}\left(n^{3}-n\right) \delta_{n,-m}\right)
$$

Likewise, the Laurent modes of the other $\operatorname{spin}-s_{i}$ operators $S^{\left(s_{i}\right)}(z)$ will generate (up to a factor of $\left(k+h^{\vee}\right)$ like in (3.20) $)$ the quantum commutator relations of a $\mathcal{W}_{N}$ algebra. Since we shall not need to refer explicitly to these relations in our following discussion, we shall omit them for brevity, as they tend to get very elaborate for $N \geq 4$.

Now let us consider the case when $k=-h^{\vee}$. From our earlier explanations about the nature of the $S^{\left(s_{i}\right)}(z)$ operators, we find that they will cease to exist as quantum operators 
at $k=-h^{\vee}$. Since we understand that the $S^{\left(s_{i}\right)}(z)$ 's must be holomorphic classical fields at $k=-h^{\vee}$, we shall rewrite the Laurent expansion of $S^{\left(s_{i}\right)}(z)$ as

$$
S^{\left(s_{i}\right)}(z)=\sum_{n \in \mathbb{Z}} S_{n}^{\left(s_{i}\right)} z^{-n-s_{i}}
$$

so as to differentiate the classical modes of expansion $S_{n}^{\left(s_{i}\right)}$ from their quantum counterpart $\hat{S}_{n}^{\left(s_{i}\right)}$ in (3.19). Unlike the $\hat{S}_{n}^{\left(s_{i}\right)}$ 's which obey the quantum commutator relations of a $\mathcal{W}_{N^{-}}$ algebra for an arbitrary level $k \neq-h^{\vee}$, the $S_{n}^{\left(s_{i}\right)}$ 's, being the modes of a Laurent expansion of a classical field, will instead obey Poisson bracket relations that define a certain classical algebra at $k=-h^{\vee}$. Since every $\hat{S}_{n}^{\left(s_{i}\right)}$ must reduce to its classical counterpart $S_{n}^{\left(s_{i}\right)}$ at $k=-h^{\vee}$, one can see that by taking $\left(k+h^{\vee}\right) \rightarrow 0$, we are actually going to the classical limit. This is analogous to taking the $\hbar \rightarrow 0$ limit in any quantum mechanical theory whenever one wants to ascertain its classical counterpart. In fact, by identifying $\left(k+h^{\vee}\right)$ with $i \hbar$, and by noting that one must make the replacement from Possion brackets to commutators via $\left\{E_{n}^{\left(s_{i}\right)}, E_{m}^{\left(s_{j}\right)}\right\}_{P . B .} \rightarrow \frac{1}{i \hbar}\left[\hat{E}_{n}^{\left(s_{i}\right)}, \hat{E}_{m}^{\left(s_{j}\right)}\right]$ in quantising any classical mode $E_{n}^{\left(s_{i}\right)}$ into an operator

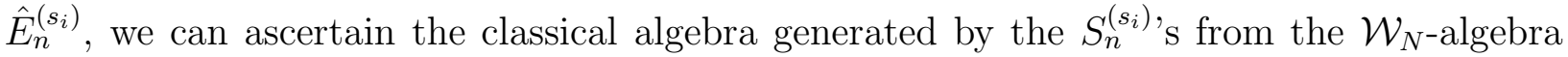
commutator relations that they satisfy. Since the $S^{\left(s_{i}\right)}(z)$ fields all lie in the classical $\bar{Q}_{+}{ }^{-}$ cohomology of the twisted sigma-model on $S L(N) / B$, it will mean that their Laurent modes $S_{n}^{\left(s_{i}\right)}$ will generate a closed, classical algebra as well 11 In fact, they will generate a closed classical $\mathcal{W}_{N}$-algebra. In order to ascertain the central charge of this classical $\mathcal{W}_{N}$-algebra, it suffices to determine the central charge of its classical Virasoro subalgebra generated by the $S_{m}^{(2)}$ 's. From (3.20), we find that as $k \rightarrow-h^{\vee}$, the $S_{m}^{(2)}$ 's satisfy

$$
\left\{S_{n}^{(2)}, S_{m}^{(2)}\right\}_{P . B .}=(n-m) S_{n+m}^{(2)}-\frac{h^{\vee} \operatorname{dim} \mathfrak{s l}_{N}}{12}\left(n^{3}-n\right) \delta_{n,-m}
$$

the classical Virasoro algebra with central charge $c=-h^{\vee} \operatorname{dim}\left(\mathfrak{s l}_{N}\right)$. Hence, the $S_{n}^{\left(s_{i}\right)}$ 's will generate a classical $\mathcal{W}_{N}$-algebra with central charge $c=-h^{\vee} \operatorname{dim}\left(\mathfrak{s l}_{N}\right)$. Indeed for the case of $\mathfrak{g}=\mathfrak{s l}_{2}$ as analysed in $\S 2.2$, the modes $S_{m}^{(2)}$ was shown to generate a classical $\mathcal{W}_{2}$-algebra with central charge $c=-h^{\vee} \operatorname{dim}\left(\mathfrak{s l}_{2}\right)=-6$, where $h^{\vee}=2$ and $\operatorname{dim}\left(\mathfrak{s l}_{2}\right)=3$. Likwise for the case of $\mathfrak{g}=\mathfrak{s l}_{3}$ as analysed in $\S 2.3$, the modes $S_{m}^{(2)}$ and $S_{m}^{(3)}$ was shown to generate a classical $\mathcal{W}_{3}$-algebra with central charge $c=-h^{\vee} \operatorname{dim}\left(\mathfrak{s l}_{2}\right)=-24$, where $h^{\vee}=3$ and $\operatorname{dim}\left(\mathfrak{s l}_{3}\right)=8$.

\footnotetext{
${ }^{11}$ Note at this point that if $\mathcal{O}$ and $\mathcal{O}^{\prime}$ are non-exact, $Q$-closed observables in some $Q$-cohomology, i.e., $\{Q, \mathcal{O}\}=\left\{Q, \mathcal{O}^{\prime}\right\}=0$, then $\left\{Q, \mathcal{O O}^{\prime}\right\}=0$. Moreover, if $\{Q, \mathcal{O}\}=0$, then $\mathcal{O}\{Q, W\}=\{Q, \mathcal{O} W\}$ for any observable $W$. These two statements mean that the cohomology classes of observables that commute with $Q$ form a closed and well-defined algebra.
} 
Last but not least, recall that the Laurent modes of the $S^{\left(s_{i}\right)}(z)$ fields for $i=1,2, \ldots$ $\operatorname{rank}\left(\mathfrak{s l}_{N}\right)$, will together generate $\mathfrak{z}\left(\widehat{\mathfrak{s l}}_{N}\right)$, the centre of the completed universal enveloping algebra of the affine $S L(N)$ algebra $\widehat{\mathfrak{s l}}_{N}$ at the critical level $k=-h^{\vee}$. If we denote the classical $\mathcal{W}_{N}$-algebra with central charge $c=-h^{\vee} \operatorname{dim}\left(\mathfrak{s l}_{N}\right)$ as $\mathcal{W}_{N}\left(-h^{\vee} \operatorname{dim}\left(\mathfrak{s l}_{N}\right)\right)$, we will have an identification of Poisson algebras $\mathfrak{z}\left(\widehat{\mathfrak{s l}}_{N}\right) \simeq \mathcal{W}_{N}\left(-h^{\vee} \operatorname{dim}\left(\mathfrak{s l}_{N}\right)\right)$.

\section{2. $\mathcal{W}$-Algebras from an Algebraic Drinfeld-Sokolov Reduction Scheme}

We shall now review a purely algebraic approach to generating $\mathcal{W}_{k^{\prime}}\left(\widehat{\mathfrak{s l}}_{N}\right)$, the $\mathcal{W}$-algebra associated to the affine $S L(N)$ algebra $\widehat{\mathfrak{s l}}_{N}$ at level $k^{\prime}$. This approach is known as the quantum Drinfeld-Sokolov (DS) reduction scheme [6, 27].

In general, the quantum DS-reduction scheme can be summarised as the following steps. Firstly, one starts with a triple $\left(\widehat{\mathfrak{g}}, \widehat{\mathfrak{g}}^{\prime}, \chi\right)$, where $\widehat{\mathfrak{g}}^{\prime}$ is an affine subalgebra of $\widehat{\mathfrak{g}}$ at level $k^{\prime}$, and $\chi$ is a 1-dimensional representation of $\widehat{\mathfrak{g}}^{\prime}$. Next, one imposes the first class constraints $g \sim \chi(g), \forall g \in \widehat{\mathfrak{g}}^{\prime}$, via a BRST procedure. The cohomology of the BRST operator $Q$ on the set of normal-ordered expressions in currents, ghosts and their derivatives, is what is called the Hecke algebra $H_{Q}^{i}\left(\widehat{\mathfrak{g}}, \widehat{\mathfrak{g}}^{\prime}, \chi\right)$ of the triple $\left(\widehat{\mathfrak{g}}, \widehat{\mathfrak{g}}^{\prime}, \chi\right)$. For generic values of $k^{\prime}$, the Hecke algebra vanishes for $i \neq 0$, and the existing zeroth cohomology $H_{Q}^{0}\left(\widehat{\mathfrak{g}}, \widehat{\mathfrak{g}}^{\prime}, \chi\right)$, is just spanned by a set of local operators associated to the triple $\left(\widehat{\mathfrak{g}}, \widehat{\mathfrak{g}}^{\prime}, \chi\right)$, whose Laurent modes generate a closed $\mathcal{W}$-algebra. We shall denote the $\mathcal{W}$-algebra associated with this set of operators as $\mathcal{W}_{D S}\left[\widehat{\mathfrak{g}}, \widehat{\mathfrak{g}}^{\prime}, \chi\right]$. Note that $\mathcal{W}_{D S}\left[\widehat{\mathfrak{s l}}_{N}, \widehat{\mathfrak{s l}}_{N}^{\prime}, \chi\right]$ is just $\mathcal{W}_{k^{\prime}}\left(\widehat{\mathfrak{s l}}_{N}\right)$, the $\mathcal{W}$-algebra associated to $\widehat{\mathfrak{s l}}_{N}$ at level $k^{\prime}$ whose quantum and classical limits we encountered in $\S 2$ for $N=2,3$. Let us be more explicit about how one can go about defining $\mathcal{W}_{D S}\left[\widehat{\mathfrak{g}}, \widehat{\mathfrak{g}}^{\prime}, \chi\right]$ and therefore $\mathcal{W}_{k^{\prime}}\left(\widehat{\mathfrak{s l}}_{N}\right)$, now that we have sketched the general idea behind the DS-reduction scheme.

In order for $\mathcal{W}_{D S}\left[\widehat{\mathfrak{g}}, \widehat{\mathfrak{g}}^{\prime}, \chi\right]$ to be a $\mathcal{W}$-algebra, one has to suitably choose the triple $\left(\widehat{\mathfrak{g}}, \widehat{\mathfrak{g}}^{\prime}, \chi\right)$. A suitable triple can be obtained by considering a principal $\mathfrak{s l}_{2}$ embedding in $\mathfrak{g}$. Let us now describe this embedding. Suppose we have an $\mathfrak{s l}_{2}$ subalgebra $\left\{t_{3}, t_{+}, t_{-}\right\}$of $\mathfrak{g}$. The adjoint representation of $\mathfrak{g}$ decomposes into $\mathfrak{s l}_{2}$ representations of $\operatorname{spin} j_{k}, k=1, \ldots, s$, for example. Then, one may write the $\widehat{\mathfrak{g}}$ current $J(z)=\sum_{a}^{\operatorname{dim} \mathfrak{g}} J^{a}(z) t_{a}$ as

$$
J(z)=\sum_{k=1}^{s} \sum_{m=-j_{k}}^{j_{k}} J^{k, m}(z) t_{k, m}
$$

where $t_{k, m}$ corresponds to the generator of spin $j_{k}$ and isospin $m$ under the $\mathfrak{s l}_{2}$ subalgebra. In particular, we have the correspondences $t_{1,1}=t_{+}, t_{1,0}=t_{3}$, and $t_{1,-1}=t_{-}$. The $\mathfrak{s l}_{2}$ 
subalgebra $t_{3}, t_{+}, t_{-}$can be characterized by a "dual Weyl vector" $\rho^{\vee}$, i.e., for $\alpha \in \Delta_{+}$, where $\Delta_{+}$is the set of positive roots of $\mathfrak{g}$, we have $\left(\rho^{\vee}, \alpha\right)=1$ if and only if $\alpha$ is a simple root of $\mathfrak{g}$. The $\mathfrak{s l}_{2}$ root $\hat{\alpha}$ is given by $\hat{\alpha}=\rho /(\rho, \rho)$, and $t_{3}=\rho \cdot \mathfrak{c}$, where $\mathfrak{c}$ is the Cartan sublagebra of $\mathfrak{g}$.

Take $\widehat{\mathfrak{g}}^{\prime}$ to be the affine Lie subalgebra $\widehat{\mathfrak{n}}_{+}$generated by all $J^{k, m}(z), m>0$. Denoting the currents corresponding to positive roots $\alpha$ by $J^{\alpha}(z)$, and choosing $t_{1,1}=\sum_{i} e^{\alpha_{i}}$, one can then impose the condition (which realises the required first-class constraint $g \sim \chi(g)$ )

$$
\left.\chi_{D S}\left(J^{\alpha}(z)\right)=1 \text { (for simple roots } \alpha_{i},\right), \quad \chi\left(J^{\alpha}(z)\right)=0 \text { (otherwise). }
$$

Next, we introduce pairs of ghost fields $\left(b^{\alpha}(z), c_{\alpha}(z)\right)$, one for every positive root $\alpha \in \Delta_{+}$. By definition, they obey the OPE $b_{\alpha}(z) c_{\beta}\left(z^{\prime}\right) \sim \delta_{\alpha \beta} /\left(z-z^{\prime}\right)$, where the $\alpha, \beta$ (and $\gamma$ ) indices run over the basis of $\mathfrak{n}_{+}$. The BRST operator that is consistent with (3.24) will then be given by $Q=Q_{0}+Q_{1}$, where

$$
Q_{0}=\oint \frac{d z}{2 \pi i}\left(J^{\alpha}(z) c_{\alpha}(z)-\frac{1}{2} f_{\gamma}^{\alpha \beta}\left(b^{\gamma} c_{\alpha} c_{\beta}\right)(z)\right)
$$

is the standard differential associated to $\widehat{\mathfrak{n}}_{+}, f_{\gamma}^{\alpha \beta}$ are the structure constants of $\mathfrak{n}_{+}$, and

$$
Q_{1}=-\oint \frac{d z}{2 \pi i} \chi_{D S}\left(J^{\alpha}(z)\right) c_{\alpha}(z)
$$

They satisfy

$$
Q^{2}=Q_{0}^{2}=Q_{1}^{2}=\left\{Q_{0}, Q_{1}\right\}=0
$$

The resulting $Q$-cohomology is just the Hecke algebra $H_{Q}^{0}\left(\widehat{\mathfrak{g}}, \widehat{\mathfrak{g}}^{\prime}, \chi\right)$, which is spanned by a set of local operators whose Laurent modes generate $\mathcal{W}_{D S}\left[\widehat{\mathfrak{g}}, \widehat{\mathfrak{g}}^{\prime}, \chi\right]=\mathcal{W}_{k^{\prime}}(\widehat{\mathfrak{g}})$. Note that (3.27) implies that one can compute the Hecke algebra via a spectral sequence of a double complex with differentials being $Q_{0}$ and $Q_{1}$ accordingly - this strategy has indeed been employed in [28] to compute explicitly the generators of the $\mathcal{W}_{2}=\mathcal{W}_{k^{\prime}}\left(\widehat{\mathfrak{s l}}_{2}\right)$ and $\mathcal{W}_{3}=\mathcal{W}_{k^{\prime}}\left(\widehat{\mathfrak{s l}}_{3}\right)$ OPE algebras with central charges $c=13-6\left(k^{\prime}+2\right)-6 /\left(k^{\prime}+2\right)$ and $c=50-24\left(k^{\prime}+3\right)-24 /\left(k^{\prime}+3\right)$ respectively. We shall have more to say about these $\mathcal{W}$-algebras shortly.

The variation of the various fields under the action of $Q$ can also be computed using the OPE's of the affine algebra $\widehat{\mathfrak{g}}$, the OPE's of the ghost fields, and the explicit forms of $Q_{0}$ and $Q_{1}$ in (3.25) and (3.26) above, and they are given by

$$
\begin{aligned}
& \delta c_{\alpha}(z)=-\frac{1}{2} f_{\alpha}^{\beta \gamma}\left(c_{\beta} c_{\gamma}\right)(z), \\
& \delta b^{\alpha}(z)=J^{\alpha}(z)-\chi_{D S}\left(J^{\alpha}(z)\right)-f_{\gamma}^{\alpha \beta}\left(b^{\gamma} c_{\beta}\right)(z) .
\end{aligned}
$$


Note also that $\mathcal{W}_{D S}\left[\widehat{\mathfrak{g}}, \widehat{\mathfrak{g}}^{\prime}, \chi\right]$ and thus $\mathcal{W}_{k^{\prime}}(\widehat{\mathfrak{g}})$, will at least contain the Virasoro algebra. The explicit form of the stress tensor whose Laurent modes will generate the Virasoro algebra is (after omitting the normal-ordering symbol)

$$
T_{D S}(z)=\frac{d_{a b} J^{a}(z) J^{b}(z)}{\left(k^{\prime}+h^{\vee}\right)}+\sum_{c=1}^{\operatorname{dimc}} \partial_{z} J^{c}(z)+\sum_{\alpha \in \Delta_{+}}\left(\left(\rho^{\vee}, \alpha\right)-1\right) b^{\alpha} \partial_{z} c_{\alpha}(z)+\left(\rho^{\vee}, \alpha\right)\left(\partial_{z} b^{\alpha} c_{\alpha}\right)(z)
$$

where the $J^{c}(z)$ 's are just the affine currents that are valued in the Cartan subalgebra $\mathfrak{c}$ of the Lie algebra $\mathfrak{g}$. Note that with respect to $T_{D S}(z)$, the conformal dimensions of the pair $\left(b^{\alpha}(z), c_{\alpha}(z)\right)$ will be given by $\left(1-\left(\rho^{\vee}, \alpha\right),\left(\rho^{\vee}, \alpha\right)\right)$. The central charge of this Virasoro subalgebra and therefore that of $\mathcal{W}_{k^{\prime}}(\widehat{\mathfrak{g}})$, will be given by

$$
c=\frac{k^{\prime} \operatorname{dim} \mathfrak{g}}{\left(k^{\prime}+h^{\vee}\right)}-12 k^{\prime}\left|\rho^{\vee}\right|^{2}-2 \sum_{\alpha \in \Delta_{+}}\left(6\left(\rho^{\vee}, \alpha\right)^{2}-6\left(\rho^{\vee}, \alpha\right)+1\right)
$$

3.3. The B-Gauged WZW Model on $S L(N)$ and the Algebraic Drinfeld-Sokolov Reduction Scheme

The $\mathcal{W}_{k^{\prime}}\left(\widehat{\mathfrak{s l}}_{2}\right)$ Algebra from the DS-Reduction Scheme

Note that from (3.31), for $\mathfrak{g}=\mathfrak{s l}_{2}$, where $\operatorname{dim}\left(\mathfrak{s l}_{2}\right)=3, h^{\vee}=2, \rho^{\vee}=\rho,\left|\rho^{\vee}\right|^{2}=1 / 2$, and $\left(\rho^{\vee}, \alpha\right)=1$, we find that the central charge of the resulting algebra $\mathcal{W}_{k^{\prime}}\left(\widehat{\mathfrak{s l}}_{2}\right)$ generated by the Laurent modes of the local operators that span the $Q$-cohomology, will be given by $c=3 k^{\prime} /\left(k^{\prime}+2\right)-6 k^{\prime}-2=13-6\left(k^{\prime}+2\right)-6 /\left(k^{\prime}+2\right)$. This is exactly the $\mathcal{W}$-algebra that the Laurent modes of the $T_{\text {total }}(z)$ operator which span the holomorphic BRST-cohomology of the $B$-gauged WZW model on $S L(2)$, generate.

In addition, for $\mathfrak{g}=\mathfrak{s l}_{2}$, we have from (3.30), the stress tensor

$$
T_{D S}(z)=T_{S L(2)}+\partial_{z} J^{1}(z)+\left(\partial_{z} b^{1}\right)\left(c_{1}\right)(z)
$$

where the conformal dimensions of $\left(b^{1}, c_{1}\right)$ are $(0,1)$ respectively. Thus, we see that $T_{D S}(z)$ is exactly $T_{\text {total }}(z)$ of the $B$-gauged WZW model on $S L(2)$, and that moreover, the ghost fields $\left(b^{1}, c_{1}\right)$ of the DS-reduction scheme have the same conformal dimensions as the $\left(b^{1}, c_{z}^{1}\right)$ ghost fields of the WZW model.

The field variations $(3.28)-(3.29)$ can in this case be written, (after noting that $\mathfrak{n}_{+} \in \mathfrak{s l}_{2}$ 
is abelian and hence has vanishing structure constants), as

$$
\begin{aligned}
\delta c_{1}(z) & =0, \\
\delta b^{1}(z) & =J^{1}(z)-\chi_{D S}\left(J^{1}(z)\right) .
\end{aligned}
$$

These variations coincide exactly with the holomorphic BRST-variations in (2.52) of the $B$-gauged WZW model on $S L(2)$ after one makes an identification between the arbitrary constant $M_{+}^{1}$ and $-\chi_{D S}\left(J^{1}(z)\right)=-1$. Moreover, the BRST-charge $Q=Q_{0}+Q_{1}$ which generates the variations in (3.33)-(3.34) will be given by

$$
Q=\oint \frac{d z}{2 \pi i}\left(J^{1}(z)-\chi_{D S}\left(J^{1}(z)\right)\right) c_{1}(z)
$$

Notice that $Q$ also coincides with $Q_{\mathrm{BRST}}$ of (2.53) - the holomorphic BRST-charge of the $B$-gauged WZW model on $S L(2)$.

In summary, we find that the holomorphic BRST-cohomology of the $B$-gauged WZW model on $S L(2)$, furnishes a physical realisation of the purely algebraic DS-reduction scheme of generating the Hecke algebra associated to $\mathcal{W}_{k^{\prime}}\left(\widehat{\mathfrak{s l}}_{2}\right)$. The classical limit of $\mathcal{W}_{k^{\prime}}\left(\widehat{\mathfrak{s l}}_{2}\right)$ - given by $\mathcal{W}_{\infty}\left(\widehat{\mathfrak{s l}}_{2}\right)$ - is indeed the classical $\mathcal{W}$-algebra generated by the Laurent modes of the field $\bar{T}_{\text {classical }}(z)$ in the classical, holomorphic BRST-cohomology of the $B$-gauged WZW model on $S L(2)$.

The $\mathcal{W}_{k^{\prime}}\left(\widehat{\mathfrak{s l}}_{3}\right)$ Algebra from the DS-Reduction Scheme

Likewise, note that from (3.31), for $\mathfrak{g}=\mathfrak{s l}_{3}$, where $\operatorname{dim}\left(\mathfrak{s l}_{2}\right)=8, h^{\vee}=3, \rho^{\vee}=\rho$, $\left|\rho^{\vee}\right|^{2}=2,\left(\rho^{\vee}, \alpha_{1}\right)=1,\left(\rho^{\vee}, \alpha_{2}\right)=1$, and $\left(\rho^{\vee}, \alpha_{3}\right)=2$, we find that the central charge of the resulting algebra $\mathcal{W}_{k^{\prime}}\left(\widehat{\mathfrak{s l}}_{3}\right)$ generated by the Laurent modes of local operators in the $Q$-cohomology, will be given by $c=8 k^{\prime} /\left(k^{\prime}+3\right)-24 k^{\prime}-30=50-24\left(k^{\prime}+3\right)-24 /\left(k^{\prime}+3\right)$. This is exactly the $\mathcal{W}$-algebra that the Laurent modes of the $T_{\text {total }}(z)$ and $T_{\text {total }}^{(3)}(z)$ operators in the holomorphic BRST-cohomology of the $B$-gauged WZW model on $S L(3)$, generate.

In addition, for $\mathfrak{g}=\mathfrak{s l}_{3}$, we have from (3.30), the stress tensor

$$
T_{D S}(z)=T_{S L(3)}+\partial_{z} J^{1}(z)+\partial_{z} J^{2}(z)+\left(\partial_{z} b^{1}\right)\left(c_{1}\right)(z)+\left(\partial_{z} b^{2}\right)\left(c_{2}\right)(z)+2\left(\partial_{z} b^{3}\right)\left(c_{3}\right)(z)+\left(b^{3}\right)\left(\partial_{z} c_{3}\right)(z),
$$

where the conformal dimensions of $\left(b^{1}, c_{1}\right),\left(b^{2}, c_{2}\right)$ and $\left(b^{3}, c_{3}\right)$ are $(0,1),(0,1)$ and $(-1,2)$, respectively. Thus, we see that $T_{D S}(z)$ is exactly $T_{\text {total }}(z)$ of the $B$-gauged WZW model on $S L(3)$, and that moreover, the ghost fields $\left(b^{1}, c_{1}\right),\left(b^{2}, c_{2}\right)$ and $\left(b^{3}, c_{3}\right)$ of the DS-reduction 
scheme have the same conformal dimensions as the $\left(b^{1}, c_{z}^{1}\right),\left(b^{2}, c_{z}^{2}\right)$ and $\left(b^{3, z}, c_{z z}^{3}\right)$ ghost fields of the WZW model.

The field variations (3.28) $-(3.29)$ in this case can be written as

$$
\begin{aligned}
& \delta c_{\alpha}(z)=-\frac{1}{2} f_{\alpha}^{\beta \gamma}\left(c_{\beta} c_{\gamma}\right)(z), \\
& \delta b^{\alpha}(z)=J^{\alpha}(z)-\chi_{D S}\left(J^{\alpha}(z)\right)-f_{\gamma}^{\alpha \beta}\left(b^{\gamma} c_{\beta}\right)(z),
\end{aligned}
$$

where $\alpha, \beta, \gamma=1,2,3$. Notice that these variations coincide exactly with the holomorphic BRST-variations in (2.100) of the $B$-gauged WZW model on $S L(3)$ after one makes an identification between the arbitrary constants $M_{+}^{\alpha}$ and $-\chi_{D S}\left(J^{\alpha}(z)\right)$. Moreover, the BRSTcharge $Q=Q_{0}+Q_{1}$ which generates the variations in (3.37)-(3.38) will be given by

$$
Q=\oint \frac{d z}{2 \pi i} \sum_{\alpha, \beta, \gamma=1}^{3}\left(\left(J^{\alpha}(z)-\chi_{D S}\left(J^{\alpha}(z)\right)\right) c_{\alpha}(z)-\frac{1}{2} f_{\gamma}^{\alpha \beta}\left(b^{\gamma} c_{\alpha} c_{\beta}\right)(z)\right)
$$

Notice that $Q$ also coincides with $Q_{\mathrm{BRST}}$ of (2.106) - the holomorphic BRST-charge of the $B$-gauged WZW model on $S L(3)$.

In summary, we find that the holomorphic BRST-cohomology of the $B$-gauged WZW model on $S L(3)$, furnishes a physical realisation of the purely algebraic DS-reduction scheme of generating the Hecke algebra associated to $\mathcal{W}_{k^{\prime}}\left(\widehat{\mathfrak{s l}}_{3}\right)$. The classical limit of $\mathcal{W}_{k^{\prime}}\left(\widehat{\mathfrak{s l}}_{3}\right)$ - given by $\mathcal{W}_{\infty}\left(\widehat{\mathfrak{s l}}_{3}\right)$ - is indeed the classical $\mathcal{W}$-algebra generated by the Laurent modes of the fields $\bar{T}_{\text {classical }}(z)$ and $\bar{T}_{\text {classical }}^{(3)}(z)$ in the classical, holomorphic BRST-cohomology of the $B$-gauged WZW model on $S L(3)$.

The B-Gauged WZW Model on $S L(N)$ and the $\mathcal{W}_{k^{\prime}}\left(\widehat{\mathfrak{s l}}_{N}\right)$ Algebra

As one might have already guessed, the above observations about the physical realisation of the algebraic DS-reduction scheme via the holomorphic BRST-cohomology of a $B$-gauged WZW model on $S L(N)$, is actually valid for all $N$, not just $N=2,3$. Let us substantiate this statement now with a discussion of the BRST-quantisation of the $B$-gauged WZW model on $S L(N)$, which, for the cases of $S L(2)$ and $S L(3)$, we have already described explicitly in $\S 2$.

Recall from (2.43) in $\S 2.2$ that the action of the $B$-gauged WZW model on $S L(N)$ takes the form

$$
\begin{gathered}
S_{\text {B-gauged }}\left(g, A_{z}, A_{\bar{z}}, J^{+}, \bar{J}^{+}\right)=S_{\mathrm{WZ}}(g)-\frac{k^{\prime}}{2 \pi} \int_{\Sigma} d^{2} z \operatorname{Tr}\left[A_{\bar{z}}\left(J^{+}(z)+M^{+}\right)-A_{z}\left(\bar{J}^{+}(\bar{z})+\bar{M}^{+}\right)\right. \\
\left.-A_{z} g A_{\bar{z}} g^{-1}+A_{z} A_{\bar{z}}\right] .
\end{gathered}
$$


As explained in $\S 2.2$, with respect to the Cartan decomposition $\mathfrak{s l}_{N}=\mathfrak{n}_{-} \oplus \mathfrak{c} \oplus \mathfrak{n}_{+}$, one can write $J(z)=\sum_{a=1}^{\operatorname{dimn}_{-}} J_{-}^{a}(z) t_{a}^{-}+\sum_{a=1}^{\operatorname{dim} \mathfrak{c}} J_{c}^{a}(z) t_{a}^{c}+\sum_{a=1}^{\operatorname{dim} \mathfrak{n}_{+}} J_{+}^{a}(z) t_{a}^{+}, \bar{J}(\bar{z})=\sum_{a=1}^{\operatorname{dimn}_{-}} \bar{J}_{-}^{a}(\bar{z}) t_{a}^{-}+$ $\sum_{a=1}^{\operatorname{dim} \mathfrak{c}} \bar{J}_{c}^{a}(\bar{z}) t_{a}^{c}+\sum_{a=1}^{\operatorname{dimn}_{+}} \bar{J}_{+}^{a}(\bar{z}) t_{a}^{+}, A_{z}=\sum_{a=1}^{\operatorname{dim} \mathfrak{n}_{+}} \tilde{A}_{z}^{a} t_{a}^{+}$and $A_{\bar{z}}=\sum_{a=1}^{\operatorname{dim} \mathfrak{n}_{+}} \tilde{A}_{\bar{z}}^{a} t_{a}^{+}$, where $t_{a}^{-} \in \mathfrak{n}_{-}$, $t_{a}^{c} \in \mathfrak{c}$, and $t_{a}^{+} \in \mathfrak{n}_{+}$. One can also write $M=\sum_{a=1}^{\operatorname{dimn}_{-}} M_{-}^{a} t_{a}^{-}+\sum_{a=1}^{\operatorname{dimc}} M_{c}^{a} t_{a}^{c}+\sum_{a=1}^{\operatorname{dimn}_{+}} M_{+}^{a} t_{a}^{+}$, where $M_{ \pm ; c}^{a}$ are arbitrary number constants, and one can also write $\bar{M}=\sum_{a=1}^{\text {dimn }_{-}} \bar{M}_{-}^{a} t_{a}^{-}+$ $\sum_{a=1}^{\operatorname{dimc}} \bar{M}_{c}^{a} t_{a}^{c}+\sum_{a=1}^{\operatorname{dimn}_{+}} \bar{M}_{+}^{a} t_{a}^{+}$, where $\bar{M}_{ \pm ; c}^{a}$ are arbitrary number constants. Then, one can write (3.40) as

$$
\begin{gathered}
S_{S L(N)}\left(g, A_{z}, A_{\bar{z}}, J^{+}, \bar{J}^{+}\right)=S_{\mathrm{WZ}}(g)-\frac{k^{\prime}}{2 \pi} \int_{\Sigma} d^{2} z \sum_{l=1}^{\operatorname{dimn} \mathfrak{n}_{+}}\left[\tilde{A}_{\bar{z}}^{l}\left(J_{+}^{l}(z)+M_{+}^{l}\right)-\tilde{A}_{z}^{l}\left(\bar{J}_{+}^{l}(\bar{z})+\bar{M}_{+}^{l}\right)\right] \\
-\operatorname{Tr}\left[A_{z} g A_{\bar{z}} g^{-1}-A_{z} A_{\bar{z}}\right]
\end{gathered}
$$

Due to the $B$-gauge invariance of the theory, we must divide the measure in any path integral computation by the volume of the $B$-gauge symmetry. That is, the partition function has to take the form

$$
Z_{S L(N)}=\int_{\Sigma} \frac{\left[g^{-1} d g, d \tilde{A}_{z}^{l}, d \tilde{A}_{\bar{z}}^{l}\right]}{\text { (gauge volume) }} \exp \left(i S_{S L(N)}\left(g, A_{z}, A_{\bar{z}}, J^{+}, \bar{J}^{+}\right)\right)
$$

One must now fix this gauge invariance to eliminate the non-unique degrees of freedom. One can do this by employing the BRST formalism which requires the introduction of Faddev-Popov ghost fields. In order to obtain the holomorphic BRST transformations of the fields, one simply replaces the infinitesimal position-dependent parameters $\epsilon^{l}$ of $h=B=$ $\exp \left(-\sum_{l=1}^{\operatorname{dimn}_{+}} \epsilon^{l} t_{m}^{+}\right)$in the corresponding left-sector of the gauge transformations in (2.34) with the ghost fields $c^{l}$, which then gives us

$$
\delta_{\mathrm{BRST}}(g)=-c^{l} t_{l}^{+} g, \quad \delta_{\mathrm{BRST}}\left(\tilde{A}_{\bar{z}}^{l}\right)=-D_{\bar{z}} c^{l}, \quad \delta_{\mathrm{BRST}}(\text { others })=0 .
$$

The components of the ghost field $c(z)=\sum_{l=1}^{\operatorname{dimn} n_{+}} c^{l}(z) t_{l}^{+}$and those of its anti-ghost partner $b(z)=\sum_{l=1}^{\operatorname{dimn}_{+}} b^{l}(z) t_{l}^{+}$will transform as

$$
\delta_{\mathrm{BRST}}\left(c^{l}\right)=-\frac{1}{2} f_{m k}^{l} c^{m} c^{k}, \quad \delta_{\mathrm{BRST}}\left(b^{l}\right)=\tilde{B}^{l}, \quad \delta_{\mathrm{BRST}}\left(\tilde{B}^{l}\right)=0,
$$

where the $f_{m k}^{l}$ 's are the structure constants of the nilpotent subalgebra $\mathfrak{n}_{+}$. Also, the $\tilde{B}^{l}$ 's are the Nakanishi-Lautrup auxiliary fields that are the BRST transforms of the $b^{l}$ 's. They also serve as Lagrange multipliers to impose the gauge-fixing conditions. 
In order to obtain the antiholomorphic BRST transformations of the fields, one employs the same recipe to the corresponding right-sector of the gauge transformations in (2.34) with the infinitesimal position-dependent gauge parameter now replaced by the ghost field $\bar{c}^{l}$, which then gives us

$$
\bar{\delta}_{\mathrm{BRST}}(g)=\bar{c}^{l} t_{l}^{+} g, \quad \bar{\delta}_{\mathrm{BRST}}\left(\tilde{A}_{z}^{l}\right)=-D_{z} \bar{c}^{l}, \quad \bar{\delta}_{\mathrm{BRST}}(\text { others })=0
$$

The components of the ghost field $\bar{c}(\bar{z})=\sum_{l=1}^{\operatorname{dim}_{+}} \bar{c}^{l}(\bar{z}) t_{l}^{+}$and those of its anti-ghost partner $\bar{b}(\bar{z})=\sum_{l=1}^{\operatorname{dimn}_{+}} \bar{b}^{l}(\bar{z}) t_{l}^{+}$will transform as

$$
\bar{\delta}_{\mathrm{BRST}}\left(\bar{c}^{l}\right)=-\frac{1}{2} f_{m k}^{l} \bar{c}^{m} \bar{c}^{k}, \quad \bar{\delta}_{\mathrm{BRST}}\left(\bar{b}^{l}\right)=\tilde{\bar{B}}^{l}, \quad \bar{\delta}_{\mathrm{BRST}}\left(\tilde{\bar{B}}^{l}\right)=0 .
$$

In the above, the $\tilde{\bar{B}}^{l}$ 's are the Nakanishi-Lautrup auxiliary fields that are the antiholomorphic BRST transforms of the $\bar{b}^{l}$ fields. They also serve as Lagrange multipliers to impose the gauge-fixing conditions.

Since the BRST transformations in (3.43) and (3.45) are just infinitesimal versions of the gauge transformations in (2.34),$S_{S L(N)}\left(g, A_{z}, A_{\bar{z}}, J^{+}, \bar{J}^{+}\right)$will be invariant under them. As in the $S L(2)$ and $S L(3)$ cases, in addition to $\left(\delta_{\mathrm{BRST}}+\bar{\delta}_{\mathrm{BRST}}\right) \cdot\left(\delta_{\mathrm{BRST}}+\bar{\delta}_{\mathrm{BRST}}\right)=0$, the holomorphic and antiholomorphic BRST-variations are also separately nilpotent, i.e., $\delta_{\mathrm{BRST}}^{2}=0$ and $\bar{\delta}_{\mathrm{BRST}}^{2}=0$. Moreover, $\delta_{\mathrm{BRST}} \cdot \bar{\delta}_{\mathrm{BRST}}=-\bar{\delta}_{\mathrm{BRST}} \cdot \delta_{\mathrm{BRST}}$. This means that the BRST-cohomology of the $B$-gauged WZW model on $S L(N)$ can be decomposed into independent holomorphic and antiholomorphic sectors that are just complex conjugate of each other, and that it can be computed via a spectral sequence, whereby the first two complexes will be furnished by its holomorphic and antiholomorphic BRST-cohomologies respectively. Since we will only be interested in the holomorphic chiral algebra of the $B$ gauged WZW model on $S L(N)$ (which as mentioned, is just identical to its antiholomorphic chiral algebra by a complex conjugation), we shall henceforth focus on the holomorphic BRST-cohomology of the $B$-gauged WZW model on $S L(N)$.

By the usual recipe of the BRST formalism, one can fix the gauge by adding to the BRST-invariant action $S_{S L(N)}\left(g, A_{z}, A_{\bar{z}}, J^{+}, \bar{J}^{+}\right)$, a BRST-exact term. Since the BRST transformation by $\left(\delta_{\mathrm{BRST}}+\bar{\delta}_{\mathrm{BRST}}\right)$ is nilpotent, the new total action will still be BRST-invariant as required. The choice of the BRST-exact operator will then define the gauge-fixing conditions. A consistent choice of the BRST-exact operator that will give us the requisite action 
for the ghost and anti-ghost fields is

$$
S_{S L(N)}\left(g, A_{z}, A_{\bar{z}}, J^{+}, \bar{J}^{+}\right)+\left(\delta_{\mathrm{BRST}}+\bar{\delta}_{\mathrm{BRST}}\right)\left(\frac{k^{\prime}}{2 \pi} \int_{\Sigma} d^{2} z \sum_{l=1}^{\operatorname{dim} \mathfrak{n}_{+}} \tilde{A}_{\bar{z}}^{l} b^{l}+\tilde{A}_{z}^{l} \bar{b}^{l}\right)
$$

where one will indeed have the desired total action, which can be written as

$$
\begin{array}{r}
S_{\mathrm{WZW}}(g)-\frac{k^{\prime}}{2 \pi} \int_{\Sigma} d^{2} z\left\{\sum_{l=1}^{\mathrm{dim} \mathfrak{n}_{+}}\left[\tilde{A}_{\bar{z}}^{l}\left(J_{+}^{l}(z)+M_{+}^{l}-\tilde{B}^{l}\right)-\tilde{A}_{z}^{l}\left(\bar{J}_{+}^{l}(\bar{z})+\bar{M}_{+}^{l}+\tilde{\bar{B}}^{l}\right)\right]\right. \\
\left.-\operatorname{Tr}\left[A_{z} g A_{\bar{z}} g^{-1}-A_{z} A_{\bar{z}}\right]\right\}+\frac{k^{\prime}}{2 \pi} \int_{\Sigma} d^{2} z \sum_{l=1}^{\operatorname{dim} \mathfrak{n}_{+}}\left(c^{l} D_{\bar{z}} b^{l}++\bar{c}^{l} D_{z} \bar{b}^{l}\right) .
\end{array}
$$

From the equations of motion by varying the $\tilde{B}^{l}$ 's, we have the conditions $\tilde{A}_{\bar{z}}^{l}=0$ for $l=1, \ldots, \operatorname{dimn}_{+}$. From the equations of motion by varying the $\tilde{\bar{B}}^{l}$ 's, we also have the conditions $\tilde{A}_{z}^{l}=0$ for $l=1, \ldots$, dimn $\mathfrak{n}_{+}$. Thus, the partition function of the $B$-gauged WZW model can also be expressed as

$Z_{S L(N)}=\int\left[g^{-1} d g, d b, d c, d \bar{b}, d \bar{c}\right] \exp \left(i S_{\mathrm{WZW}}(g)+\frac{i k^{\prime}}{2 \pi} \int_{\Sigma} d^{2} z \operatorname{Tr}\left(c \cdot \partial_{\bar{z}} b\right)(z)+\operatorname{Tr}\left(\bar{c} \cdot \partial_{z} \bar{b}\right)(\bar{z})\right)$

where the holomorphic BRST variations of the fields which leave the effective action in (3.48) invariant are now given by

$$
\begin{aligned}
& \delta_{\mathrm{BRST}}(g)=-c^{m} t_{m}^{+} g, \quad \delta_{\mathrm{BRST}}\left(c^{l}\right)=-\frac{1}{2} f_{m k}^{l} c^{m} c^{k}, \quad \delta_{\mathrm{BRST}}\left(b^{l}\right)=J_{+}^{l}+M_{+}^{l}-f_{m k}^{l} b^{m} c^{k}, \\
& \delta_{\mathrm{BRST}}(\text { others })=0 .
\end{aligned}
$$

The holomorphic BRST-charge generating the field variations in (3.49) will be given by

$$
Q_{\mathrm{BRST}}=\oint \frac{d z}{2 \pi i}\left(\sum_{l=1}^{\mathrm{dimn}_{+}} c^{l}(z)\left(J_{+}^{l}(z)+M_{+}^{l}\right)-\frac{1}{2} \sum_{l=1}^{\mathrm{dim} \mathfrak{n}_{+}} f_{m k}^{l} b^{m} c^{l} c^{k}(z)\right) .
$$

The free-field action of the left-moving ghost fields in (3.48) implies that we have the usual OPE's of $\left(\operatorname{dimn}_{+}\right)$free $b c$ systems. From these free $b c$ OPE's, one can verify that $Q_{\mathrm{BRST}}$ in (3.50) will indeed generate the field variations in (3.49).

Again, though we did not make this obvious in our discussion above, by integrating out the $\tilde{A}_{\bar{z}}^{l}$ 's in (3.41), and using the above conditions $\tilde{A}_{z}^{l}=0$ for $l=1, \ldots, \operatorname{dim} \mathfrak{n}_{+}$, we 
find that we actually have the relations $\left(J_{+}^{l}(z)+M_{+}^{l}\right)=0$ for $l=1, \ldots$, dimn $\mathfrak{n}_{+}$. These relations (involving the current associated to the Borel subalgebra $\mathfrak{b}$ of the group $B$ that we are modding out by) will lead us directly to the correct form of the holomorphic stress tensor for the gauged WZW model without reference to a coset formalism.

Note that as in the $S L(2)$ and $S L(3)$ cases of $\S 2$, physically consistent with the holomorphic chiral algebra of the purely bosonic sector of the twisted sigma-model on $S L(N) / B$, there are currents $J^{a}(z)$ in the holomorphic BRST-cohomology of the non-dynamically $B$ gauged WZW model on $S L(N)$, where $a=1,2, \ldots, \operatorname{dimsl}_{N}$, that generate an affine $S L(N)$ OPE algebra at level $k^{\prime}$. As such, one can construct a holomorphic stress tensor using the Sugawara formalism as

$$
T_{S L(N)}(z)=\frac{: d_{a b}\left(J^{a} J^{b}\right)(z):}{k^{\prime}+h^{\vee}}
$$

However, as shown above, one will have the conditions $J_{+}^{l}=-M_{+}^{l}$ for $l=1,2, \ldots$, dimn . In order that the conformal dimensions of the $J_{+}^{l}$ 's be compatible with these conditions, one must define a modified holomorphic stress tensor:

$$
T_{\text {modified }}(z)=T_{S L(N)}(z)+\vec{l} \cdot \partial \vec{J}_{c}(z)
$$

where $\vec{J}_{c}(z)$ is an $(N-1)$-dimensional vector with components being the $J_{c}^{l}$ currents associated to the Cartan subalgebra $\mathfrak{c}$, and $\vec{l}$ is a sum of simple, positive roots of $\mathfrak{s l}_{N}$. In order for the above conditions on the $J_{+}^{l}$ 's be compatible with the fact that $Q_{\mathrm{BRST}}$ generating the holomorphic variations $\delta_{\mathrm{BRST}}\left(b^{l}\right)$ of (3.49) must be a scalar of dimension zero, the $\left(\operatorname{dim} \mathfrak{n}_{+}\right)$-set of left-moving ghost systems $\left(b^{l}, c^{l}\right)$ must have conformal dimensions $(1-h, h)$ for $l=1,2, \ldots, \operatorname{dimn}_{+}$, where $h$ is the conformal dimension of the corresponding $J_{+}^{l}$ current under $T_{\text {modified }}(z)$. With all these points in mind, and by including the holomorphic stress tensor contribution from the action of the left-moving ghost fields, we can write the total holomorphic stress tensor of the $B$-gauged WZW model on $S L(N)$ as

$$
T_{\text {total }}(z)=\frac{: d_{a b}\left(J^{a} J^{b}\right)(z):}{k^{\prime}+h^{\vee}}+\sum_{a=1}^{\operatorname{dim} \mathfrak{c}} \partial_{z} J_{c}^{a}(z)+\sum_{l \in \Delta_{+}}\left(\left(\rho^{\vee}, l\right)-1\right) b^{l} \partial_{z} c^{l}(z)+\left(\rho^{\vee}, l\right)\left(\partial_{z} b^{l} c^{l}\right)(z),
$$

where $\Delta_{+}$is the set of positive roots of $\mathfrak{s l}_{N}$, and $\rho^{\vee}$ is the "dual Weyl vector" of $\mathfrak{s l}_{N}$. Notice that $T_{\text {total }}(z)$ is just $T_{D S}(z)$ in (3.30) for $\widehat{\mathfrak{g}}=\widehat{\mathfrak{s l}}_{N}$. Moreover, the central charge of $T_{\text {total }}(z)$ will be given by

$$
c=N^{4}-1-N\left(N^{2}-1\right)\left(\frac{1}{k^{\prime}+N}+k^{\prime}+N\right)-\left(N^{4}-2 N^{3}+N\right),
$$


which can be rewritten as

$$
c=\frac{k^{\prime} \operatorname{dim}\left(\mathfrak{s l}_{N}\right)}{\left(k^{\prime}+h^{\vee}\right)}-12 k^{\prime}\left|\rho^{\vee}\right|^{2}-2 \sum_{l \in \Delta_{+}}\left(6\left(\rho^{\vee}, l\right)^{2}-6\left(\rho^{\vee}, l\right)+1\right),
$$

since $h^{\vee}=N$ for $\mathfrak{s l}_{N}$, and $\left(N^{2}-1\right)=\operatorname{dim}\left(\mathfrak{s l}_{N}\right)$. This coincides with the central charge of $T_{D S}(z)$ in (3.31) for $\mathfrak{g}=\mathfrak{s l}_{N}$.

Note also that for any $J_{+}^{l}$ with $h \neq 0$, the corresponding $M_{+}^{l}$ constant must be set to zero for consistency. This means that we can identify $M_{+}^{l}$ with $-\chi_{D S}\left(J_{+}^{l}(z)\right)$. Hence, we can write the holomorphic BRST-variations in (3.49) as

$$
\delta_{\mathrm{BRST}}(g)=-c^{m} t_{m}^{+} g, \quad \delta_{\mathrm{BRST}}\left(c^{l}\right)=-\frac{1}{2} f_{m k}^{l} c^{m} c^{k}, \quad \delta_{\mathrm{BRST}}\left(b^{l}\right)=J_{+}^{l}-\chi_{D S}\left(J_{+}^{I}\right)-f_{m k}^{l} b^{m} c^{k},
$$

which just coincides with the BRST-variations of the DS-reduction scheme in (3.28)-(3.29) for $\mathfrak{g}=\mathfrak{s l}_{N}$. Last but not least, the holomorphic BRST-charge which generates these field transformations can also be written as

$$
Q_{\mathrm{BRST}}=\oint \frac{d z}{2 \pi i}\left(\sum_{l=1}^{\mathrm{dimn}_{+}} c^{l}(z)\left(J_{+}^{l}(z)-\chi_{D S}\left(J_{+}^{l}(z)\right)\right)-\frac{1}{2} \sum_{l=1}^{\text {dimn }_{+}} f_{m k}^{l} b^{m} c^{l} c^{k}(z)\right) .
$$

This just coincides with the sum of $Q_{0}$ and $Q_{1}$ in (3.25) and (3.26), i.e., it coincides with $Q$ of the DS-reduction scheme for $\mathfrak{g}=\mathfrak{s l}_{N}$.

In summary, we find that the holomorphic BRST-cohomology of the $B$-gauged WZW model on $S L(N)$, will indeed furnish a physical realisation of the purely algebraic DSreduction scheme of generating the Hecke algebra that is spanned by local operators whose Laurent modes generate a $\mathcal{W}_{k^{\prime}}\left(\widehat{\mathfrak{s}}_{N}\right)$ algebra with central charge (3.31). Consequently, the classical limit of $\mathcal{W}_{k^{\prime}}\left(\widehat{\mathfrak{s l}}_{N}\right)$, i.e., $\mathcal{W}_{\infty}\left(\widehat{\mathfrak{s l}}_{N}\right)$, will be given by the Poisson $\mathcal{W}$-algebra generated by the Laurent modes of the classical counterparts of the local operators which lie in the classical, holomorphic BRST-cohomology of the $B$-gauged WZW model on $S L(N)$. We shall discuss this set of classical fields next, and their role in an isomorphism of classical $\mathcal{W}$-algebras and a level relation that underlie a geometric Langlands correspondence for any $G=S L(N)$.

\subsection{An Equivalence of Classical Holomorphic Chiral Algebras and a Geometric Langlands Correspondence for Any $S L(N)$}

Via a straightforward extension of our arguments in $\S 2$ on the $S L(2)$ and $S L(3)$ cases 
to all $S L(N)$, we find that the equivalence - at the level of the holomorphic chiral algebra - between the purely bosonic sector of the twisted sigma-model on $S L(N) / B$ and the $B$-gauged WZW model on $S L(N)$, will imply an isomorphism between the closed classical algebra generated by the Laurent modes of the $S^{\left(s_{i}\right)}(z)$ fields in the classical, holomorphic chiral algebra of the $\psi^{\bar{j}}$-independent sector of the twisted sigma-model on $S L(N) / B$, and the closed classical algebra generated by the Laurent modes of the corresponding $\bar{T}_{\text {classical }}^{\left(s_{i}\right)}(z)=$ $-h^{\vee} \cdot T_{\text {classical }}^{\left(s_{i}\right)}(z)$ fields in the classical, holomorphic BRST-cohomology of the $B$-gauged WZW model on $S L(N)$. Here, the $\bar{T}_{\text {classical }}^{\left(s_{i}\right)}(z)=\sum_{n} \bar{L}_{n}^{\left(s_{i}\right)} z^{-n-s_{i}}$ fields are just the classical counterparts of the $\bar{T}_{\text {total }}^{\left(s_{i}\right)}(z)=\left(k+h^{\vee}\right) T_{\text {total }}^{\left(s_{i}\right)}(z)$ operators that exist in the quantum, holomorphic BRST-cohomology of the gauged WZW model at $k \neq-h^{\vee}$, whereby the Laurent modes of the $T_{\text {total }}^{\left(s_{i}\right)}(z)$ operators will generate the $\mathcal{W}_{k^{\prime}}\left(\widehat{\mathfrak{s l}}_{N}\right)$-algebra discussed above.

Recall from our earlier discussion that the Laurent modes of the $S^{s_{i}}(z)$ fields will generate a classical $\mathcal{W}_{N}$-algebra that contains a Virasoro subalgebra given by

$$
\left\{S_{n}^{(2)}, S_{m}^{(2)}\right\}_{P . B .}=(n-m) S_{n+m}^{(2)}-\frac{h^{\vee} \operatorname{dim} \mathfrak{s l}_{N}}{12}\left(n^{3}-n\right) \delta_{n,-m}
$$

This classical Virasoro subalgebra has central charge $c=-h^{\vee} \operatorname{dim}\left(\mathfrak{s l}_{N}\right)$. Hence, the Laurent modes $S_{n}^{\left(s_{i}\right)}$ of the $S^{\left(s_{i}\right)}(z)$ fields will generate a classical $\mathcal{W}_{N}$-algebra with central charge $c=-h^{\vee} \operatorname{dim}\left(\mathfrak{s l}_{N}\right)$, which, we had denoted earlier as $\mathcal{W}_{N}\left(-h^{\vee} \operatorname{dim}\left(\mathfrak{s l}_{N}\right)\right)$.

On the other hand, the Laurent modes of the $\bar{T}_{\text {classical }}^{\left(s_{i}\right)}(z)$ fields will generate a classical $\mathcal{W}_{\infty}\left(\widehat{\mathfrak{s l}}_{N}\right)$-algebra, which, consistent with the equivalence of the holomorphic chiral algebras of the $\psi^{\bar{j}}$-independent sector of the twisted sigma-model on $S L(N) / B$ and the $B$-gauged WZW model on $S L(N)$, is also a classical $\mathcal{W}_{N}$-algebra. Likewise, the central charge of this classical $\mathcal{W}_{\infty}$-algebra will be given by the central charge of its classical Virasoro subalgebra. Its classical Virasoro subalgebra is given by

$$
\left\{\bar{L}_{n}^{(2)}, \bar{L}_{m}^{(2)}\right\}_{P . B .}=(n-m) \bar{L}_{n+m}^{(2)}+\frac{c\left(k, k^{\prime}\right)_{k \rightarrow-h^{\vee}, k^{\prime} \rightarrow \infty}}{12}\left(n^{3}-n\right) \delta_{n,-m}
$$

where

$$
c\left(k, k^{\prime}\right)=\left(k+h^{\vee}\right)\left(N^{4}-1-N\left(N^{2}-1\right)\left(\frac{1}{\left(k^{\prime}+h^{\vee}\right)}+\left(k^{\prime}+h^{\vee}\right)\right)-\left(N^{4}-2 N^{3}+N\right)\right),
$$

and $\bar{L}_{n}^{(2)}=-h^{\vee} \cdot L_{n}^{(2)}$ corresponds to $S_{n}^{(2)}$, while $L_{n}^{(2)}$ is a Virasoro mode of the classical counterpart $T_{\text {classical }}^{(2)}(z)$ of $T_{\text {total }}^{(2)}(z)$. Therefore, the central charge of the $\mathcal{W}_{\infty}\left(\widehat{\mathfrak{s l}}_{N}\right)$-algebra 
generated by the Laurent modes of the $\bar{T}_{\text {classical }}^{\left(s_{i}\right)}(z)$ fields will be given by $c\left(k, k^{\prime}\right)$ where $k \rightarrow-h^{\vee}$ and $k^{\prime} \rightarrow \infty$.

An isomorphism between the classical $\mathcal{W}$-algebras generated by the $S_{n}^{\left(s_{i}\right)}$ 's and the

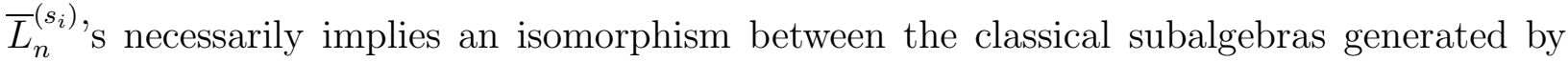
the $S_{n}^{(2)}$ 's and $\bar{L}_{n}^{(2)}$ 's in (3.58) and (3.59) respectively. This in turn means that we must have the relation

$$
c\left(k, k^{\prime}\right)_{k \rightarrow-h^{\vee}, k^{\prime} \rightarrow \infty}=-h^{\vee} \operatorname{dim} \mathfrak{s l}_{N} .
$$

In the examples studied in $\S 2$ where $N=2,3$, we saw that the above relation would hold if and only $\left(k+h^{\vee}\right)\left(k^{\prime}+h^{\vee}\right)=1$. One can quickly verify that this would also be the case for any $N$ : notice that the surviving term in $c\left(k, k^{\prime}\right)_{k \rightarrow-h^{\vee}, k^{\prime} \rightarrow \infty}$ is just $-N\left(N^{2}-1\right)\left(k+h^{\vee}\right)\left(k^{\prime}+h^{\vee}\right)$, and since $N=h^{\vee}$ and $\left(N^{2}-1\right)=\operatorname{dim}\left(\mathfrak{s l}_{N}\right)$, we will have $c\left(k, k^{\prime}\right)_{k \rightarrow-h^{\vee}, k^{\prime} \rightarrow \infty}=-h^{\vee} \operatorname{dim}\left(\mathfrak{s l}_{N}\right)$ if and only if $\left(k+h^{\vee}\right)\left(k^{\prime}+h^{\vee}\right)=1$, whence the classical $\mathcal{W}_{N}\left(-h^{\vee} \operatorname{dim}\left(\mathfrak{s l}_{N}\right)\right)$-algebra will be isomorphic to the $\mathcal{W}_{\infty}\left(\widehat{\mathfrak{s l}}_{N}\right)$-algebra with central charge $c\left(k, k^{\prime}\right)_{k \rightarrow-h^{\vee}, k^{\prime} \rightarrow \infty}=-h^{\vee} \operatorname{dim}\left(\mathfrak{s l}_{N}\right)$. Since $\mathcal{W}_{N}\left(-h^{\vee} \operatorname{dim}\left(\mathfrak{s l}_{N}\right)\right) \simeq \mathfrak{z}_{\left(\mathfrak{s l}_{N}\right)}$, and since for $\mathfrak{g}=\mathfrak{s l}_{N}={ }^{L} \mathfrak{g}, h^{\vee}={ }^{L} h^{\vee}$, and $r^{\vee}=1$, we thus see that an equivalence - at the level of the holomorphic chiral algebra - between the $\psi^{\bar{j}}$-independent sector of the twisted sigma-model on $S L(N) / B$ and the $B$-gauged WZW model on $S L(N)$, would imply an isomorphism of Poisson algebras

$$
\mathfrak{z}(\widehat{\mathfrak{g}}) \simeq \mathcal{W}_{\infty}\left({ }^{L} \widehat{\mathfrak{g}}\right)
$$

and the level relation

$$
\left(k+h^{\vee}\right) r^{\vee}=\frac{1}{\left(k^{\prime}+{ }^{L} h^{\vee}\right)} .
$$

Note at this point that the purely bosonic, $\psi^{\bar{j}}$-independent sector of the twisted sigma-model on $S L(N) / B$, can be described, via (2.31), by a bosonic string on $S L(N) / B$. On the other hand, note that since a bosonic string on a group manifold $G$ can be described as a WZW model on $G$, it will mean that the $B$-gauged WZW model on $S L(N)$ can be interpreted as a $B$-gauged bosonic string on $S L(N)$. Thus, we see that an equivalence, at the level of the holomorphic chiral algebra, between a bosonic string on $S L(N) / B$ and a $B$-gauged version of itself on $S L(N)$ - a statement which stems from the ubiquitous notion that one can always physically interpret a geometrical symmetry of the target space as a gauge symmetry in the worldsheet theory - will imply an isomorphism of classical $\mathcal{W}$-algebras and a level relation that underlie a geometric Langlands correspondence for $G=S L(N)$ ! Note that the correspondence between the $k \rightarrow-h^{\vee}$ and $k^{\prime} \rightarrow \infty$ limits (within the context of the 
above Poisson algebras) is indeed consistent with the relation (3.63). These limits define a "classical" geometric Langlands correspondence. A "quantum" generalisation of the $S L(N)$ correspondence can be defined for other values of $k$ and $k^{\prime}$ that satisfy the relation (3.63), but with the isomorphism of (3.62) replaced by an isomorphism of quantum $\mathcal{W}$-algebras (derived from a DS-reduction scheme) associated to $\widehat{\mathfrak{s l}}_{N}$ at levels $k$ and $k^{\prime}$ respectively [6].

\section{About the Hecke Eigensheaves and Hecke Operators}

We shall now demonstrate, via the isomorphism of classical $\mathcal{W}$-algebras discussed in $\S 3$, how one can derive a correspondence between flat holomorphic ${ }^{L} G$-bundles on the worldsheet $\Sigma$ and Hecke eigensheaves on the moduli space $\operatorname{Bun}_{G}$ of holomorphic $G$-bundles on $\Sigma$, where $G=S L(N)$. In the process, we will be able to physically interpret the Hecke eigensheaves and Hecke operators of the geometric Langlands program for $G=S L(N)$, in terms of the correlation functions of purely bosonic local operators in the holomorphic chiral algebra of the twisted $(0,2)$ sigma-model on the complex flag manifold $S L(N) / B$.

\subsection{Hecke Eigensheaves on Bun $_{S L(N)}$ and Flat ${ }^{L} S L(N)$-Bundles on $\Sigma$}

\section{Local Primary Field Operators}

As we will explain shortly, the correlation functions of local primary field operators can be associated to the sought-after Hecke eigensheaves. As such, let us begin by describing these operators in the twisted $(0,2)$ sigma-model on a complex flag manifold $X=S L(N) / B$. By definition, the holomorphic primary field operators $\Phi_{s}^{\lambda}(z)$ of any theory with an affine $S L(N)$ OPE algebra obey [29]

$$
J^{a}(z) \Phi_{r}^{\lambda}\left(z^{\prime}\right) \sim-\sum_{s} \frac{\left(t_{\lambda}^{a}\right)_{r s} \Phi_{s}^{\lambda}\left(z^{\prime}\right)}{z-z^{\prime}}
$$

where $t_{\lambda}^{a}$ is a matrix in the $\lambda$ representation of $\mathfrak{s l}_{N}, r, s=1, \ldots, \operatorname{dim}|\lambda|$, and $a=1, \ldots, \operatorname{dim}\left(\mathfrak{s l}_{N}\right)$.

Since the $\Phi_{s}^{\lambda}(z)$ 's obey OPE relations with the quantum operators $J^{a}(z)$, it will mean that they, like the $J^{a}(z)$ 's, must exist as quantum bosonic operators of the sigma-model on $X$. And moreover, since (4.1) and the affine $S L(N)$ OPE algebra at the critical level generated by the $J^{a}(z)$ 's in the $\bar{Q}_{+}$-cohomology of the quantum sigma-model together form a closed OPE algebra, it will mean that the $\Phi_{s}^{\lambda}(z)$ 's are also local operators in the $\bar{Q}_{+}$-cohomology of the sigma-model on $X$ at the quantum level. From our $\bar{Q}_{+}$-Cech cohomology dictionary, 
this means that the $\Phi_{s}^{\lambda}(z)$ 's will correspond to classes in $H^{0}\left(X, \mathcal{O}_{X}^{c h}\right)$, i.e., the global sections of the sheaf $\mathcal{O}_{X}^{c h}$ of CDO's on $X$. Note that this observation is also consistent with (4.1) one can generate other global sections of the sheaf $\mathcal{O}_{X}^{c h}$ from the OPE's of existing global sections. (Recall that we did this to generate the $J_{3}(z)$ current from the OPE of the $J_{-}(z)$ and $J_{+}(z)$ currents of the affine $S L(2)$ OPE algebra when we studied the sigma-model on $S L(2) / B$ in $\S 2)$.

The fact that these operators can be described by global sections of the sheaf of CDO's on $X$ means that they reside within the purely bosonic sector of the holomorphic chiral algebra of the underlying sigma-model on $X$. As we shall see, this observation will serve as a platform for a physical interpretation of the Hecke eigensheaves.

\section{Space of Coinvariants}

Associated to the correlation functions of the above-described local primary field operators, is the concept of a space of coinvariants, which, in its interpretation as a sheaf over the moduli space of holomorphic $G$-bundles on $\Sigma$ that we will clarify below, is directly related to the Hecke eigensheaves that we are looking for. Hence, let us now turn our attention to describing this space of coinvariants.

Notice that if the twisted sigma-model were to be conformal, i.e., $\left\{\bar{Q}_{+}, T_{z z}\right\}=0$ even after quantum corrections, we would have a CFT operator-state isomorphism, such that any primary field operator $\Phi_{s}^{\lambda}(z)$ would correspond to a state $\left|\Phi_{s}^{\lambda}\right\rangle$ in the highest-weight representation of $\widehat{\mathfrak{g}}=\widehat{\mathfrak{s l}}_{N}$. However, since the twisted sigma-model on a complex flag manifold $S L(N) / B$ lacks a holomorphic stress tensor and is thus non-conformal, a $\Phi_{s}^{\lambda}(z)$ operator will not have a one-to-one correspondence with a state $\left|\Phi_{s}^{\lambda}\right\rangle$. Rather, the states just furnish a module of the chiral algebra spanned by the operators themselves.

Nevertheless, in the axiomatic CFT framework of a theory with an affine algebra $\widehat{\mathfrak{g}}$, the operator-state isomorphism is an axiom that is defined at the outset, and therefore, any primary field operator will be axiomatically associated to a state in the highest-weight representation of $\widehat{\mathfrak{g}}$. Bearing this in mind, now consider a general correlation function of $n$ primary field operators such as $\left\langle\Phi_{s}^{\lambda_{1}}\left(z_{1}\right) \ldots \Phi_{s}^{\lambda_{n}}\left(z_{n}\right)\right\rangle$. Note that it can be viewed, in the axiomatic CFT sense, as a map from a tensor product of $n$ highest-weight representations of $\widehat{\mathfrak{s l}}_{N}$ to a complex number. Next, consider a variation of the correlation function under a global $S L(N)$ transformation, i.e., $\delta_{\omega}\left\langle\Phi_{s}^{\lambda_{1}}\left(z_{1}\right) \ldots \Phi_{s}^{\lambda_{n}}\left(z_{n}\right)\right\rangle=\oint_{C} d z \sum_{a} \omega^{a}\left\langle J^{a}(z) \Phi_{s}^{\lambda_{1}}\left(z_{1}\right) \ldots \Phi_{s}^{\lambda_{n}}\left(z_{n}\right)\right\rangle$, where $\omega^{a}$ is a position-independent scalar transformation parameter, and where $C$ is a contour that encircles all the points $z_{1}, \ldots, z_{n}$ on $\Sigma$. Since all the $J^{a}(z)$ 's are dimension-one conserved currents in the $\bar{Q}_{+}$-cohomology of the twisted sigma-model on $S L(N) / B$, they will generate 
a symmetry of the theory. In other words, we will have $\delta_{\omega}\left\langle\Phi_{s}^{\lambda_{1}}\left(z_{1}\right) \ldots \Phi_{s}^{\lambda_{n}}\left(z_{n}\right)\right\rangle=0$, which is simply a statement of the global $S L(N)$-invariance of any theory with an affine $S L(N)$ algebra. This last statement, together with the one preceding it, means that a general correlation function of $n$ primary field operators $\left\langle\Phi_{s}^{\lambda_{1}}\left(z_{1}\right) \ldots \Phi_{s}^{\lambda_{n}}\left(z_{n}\right)\right\rangle$ will define a "conformal block" in the axiomatic CFT sense [6]. Proceeding from this mathematical definition of a "conformal block", the collection of operators $\Phi_{s}^{\lambda_{1}}\left(z_{1}\right) \ldots \Phi_{s}^{\lambda_{n}}\left(z_{n}\right)$ will define a vector $\Phi$ in the dual space of coinvariants $H_{\mathfrak{s l}_{N}}\left(\Phi_{s}^{\lambda_{1}}\left(z_{1}\right) \ldots \Phi_{s}^{\lambda_{n}}\left(z_{n}\right)\right)$, whereby the "conformal block" or correlation function $\left\langle\Phi_{s}^{\lambda_{1}}\left(z_{1}\right) \ldots \Phi_{s}^{\lambda_{n}}\left(z_{n}\right)\right\rangle$ can be computed as the square $|\Phi|^{2}$ of length of $\Phi$ with respect to a hermitian inner product on $H_{\mathfrak{s l}_{N}}\left(\Phi_{s}^{\lambda_{1}}\left(z_{1}\right) \ldots \Phi_{s}^{\lambda_{n}}\left(z_{n}\right)\right)$ [6]. All correlation functions of primary field operators can be computed once this inner product is determined.

\section{Sheaf of Coinvariants on Bun $\operatorname{SL}_{(N)}$}

As mentioned above, what will be directly related to the Hecke eigensheaves is the sheaf of coinvariants on the moduli space $\operatorname{Bun}_{G}$ of holomorphic $G$-bundles on the worldsheet $\Sigma$. Let us now describe how this sheaf of coinvariants arises. However, before we proceed, let us first explain how holomorphic $G$-bundles on $\Sigma$ can be consistently defined in the presence of an affine $G$-algebra in the sigma-model on $X=S L(N) / B$, where $G=S L(N)$ in our case.

Recall that for the sigma-model on $X=S L(N) / B$, we have the OPE

$$
J_{a}(z) J_{b}(w) \sim-\frac{N d_{a b}}{(z-w)^{2}}+\sum_{c} f_{a b}{ }^{c} \frac{J_{c}(w)}{(z-w)}
$$

where $d_{a b}$ is the Cartan-Killing metric of $\mathfrak{s l}_{N}$. Note also that since the above dimensionone current operators are holomorphic in $\Sigma$, they can be expanded in a Laurent expansion around the point $w$ on $\Sigma$ as

$$
J_{a}(z)=\sum_{n} J_{a}^{n}(w)(z-w)^{-n-1}
$$

Consequently, from the above OPE, we will have the commuator relation

$$
\left[J_{a}^{n}(w), J_{b}^{m}(w)\right]=\sum_{c} f_{a b}^{c} J_{c}^{n+m}(w)-\left(N d_{a b}\right) n \delta_{n+m, 0}
$$

such that the Lie algebra $\mathfrak{g}=\mathfrak{s l}_{N}$ generated by the zero-modes of the currents will be given by

$$
\left[J_{a}^{0}(w), J_{b}^{0}(w)\right]=\sum_{c} f_{a b}{ }^{c} J_{c}^{0}(w) .
$$


One can then exponentiate the above generators that span $\mathfrak{s l}_{N}$ to define an element of $G=$ $S L(N)$, and since these generators depend on the point $w$ in $\Sigma$, it will mean that one can, via this exponential map, consistently define a non-trivial principal $G$-bundle on $\Sigma$. Moreover, this bundle will be holomorphic as the underlying generators only vary holomorphically in $w$ on the worldsheet $\Sigma$.

Let us label the above-described holomorphic $S L(N)$-bundle on $\Sigma$ as $\mathcal{P}$. Then, the space $H_{\mathfrak{s l}_{N}}\left(\Phi_{s}^{\lambda_{1}}\left(z_{1}\right) \ldots \Phi_{s}^{\lambda_{n}}\left(z_{n}\right)\right)$ of coinvariants will vary non-trivially under infinitesimal deformations of $\mathcal{P}$. As such, one can define a sheaf on coinvariants over the space of all holomorphic $S L(N)$-bundles on $\Sigma$, i.e., $\operatorname{Bun}_{S L(N)}$. Let us justify this statement next.

Firstly, note that with our description of $\mathcal{P}$ via the affine $S L(N)$-algebra of the sigmamodel on $X$, there is a mathematical theorem [7] which states that $\operatorname{Bun}_{S L(N)}$ is locally uniformized by the affine $S L(N)$-algebra. What this means is that the tangent space $T_{\mathcal{P}} \operatorname{Bun}_{S L(N)}$ to the point in $\operatorname{Bun}_{S L(N)}$ which corresponds to an $S L(N)$-bundle on $\Sigma$ labelled by $\mathcal{P}$, will be isomorphic to the space $H^{1}(\Sigma$, End $\mathcal{P})$ [7]. Moreover, deformations of $\mathcal{P}$, which correspond to displacements from this point in $\operatorname{Bun}_{S L(N)}$, are generated by an element $\eta(z)=J^{a} \eta_{a}(z)$ of the loop algebra of $\mathfrak{s l}_{N}$, where $\eta_{a}(z)$ is a position-dependent scalar deformation parameter (see $\S 17.1$ of [7] and $\S 7.3$ of [6]). With this in mind, let us again consider the $n$-point correlation function $\left\langle\Phi_{s}^{\lambda_{1}}\left(z_{1}\right) \ldots \Phi_{s}^{\lambda_{n}}\left(z_{n}\right)\right\rangle$. By inserting $\eta(z)$ into this correlation function, and computing the contour integral around the points $z_{1}, \ldots, z_{n}$, we have $\left.\delta_{\eta}\left\langle\Phi_{s}^{\lambda_{1}}\left(z_{1}\right) \ldots \Phi_{s}^{\lambda_{n}}\left(z_{n}\right)\right)\right\rangle=\left\langle\oint_{C} d z \sum_{a} \eta_{a}(z) J^{a}(z) \Phi_{s}^{\lambda_{1}}\left(z_{1}\right) \ldots \Phi_{s}^{\lambda_{n}}\left(z_{n}\right)\right\rangle$, where $C$ is a contour which encircles the points $z_{1}, \ldots, z_{n}$ on $\Sigma$, and $\delta_{\eta}\left\langle\Phi_{s}^{\lambda_{1}}\left(z_{1}\right) \ldots \Phi_{s}^{\lambda_{n}}\left(z_{n}\right)\right\rangle$ will be the variation of $\left\langle\Phi_{s}^{\lambda_{1}}\left(z_{1}\right) \ldots \Phi_{s}^{\lambda_{n}}\left(z_{n}\right)\right\rangle$ under an infinitesimal deformation of $\mathcal{P}$ generated by $\eta(z)$ (see eqn. (7.9) of [6] and also [30]). Note that this variation does not vanish, since $\eta_{a}(z)$, unlike $\omega$ earlier, is a position-dependent parameter of a local $S L(N)$-transformation. Therefore, as explained above, since the correlation function $\left\langle\Phi_{s}^{\lambda_{1}}\left(z_{1}\right) \ldots \Phi_{s}^{\lambda_{n}}\left(z_{n}\right)\right\rangle$ is associated to $\Phi$ in the dual space of coinvariants $H_{\mathfrak{s l}_{N}}\left(\Phi_{s}^{\lambda_{1}}\left(z_{1}\right) \ldots \Phi_{s}^{\lambda_{n}}\left(z_{n}\right)\right)$, one can see that $\Phi$ must vary in $H_{\mathfrak{s l}_{N}}\left(\Phi_{s}^{\lambda_{1}}\left(z_{1}\right) \ldots \Phi_{s}^{\lambda_{n}}\left(z_{n}\right)\right)$ as one moves infinitesimally along a path in $\operatorname{Bun}_{S L(N)}$. Since $\Phi$ is just a vector in some basis of $H_{\mathfrak{s l}_{N}}\left(\Phi_{s}^{\lambda_{1}}\left(z_{1}\right) \ldots \Phi_{s}^{\lambda_{n}}\left(z_{n}\right)\right)$, one could instead interpret $\Phi$ to be fixed, while $H_{\mathfrak{s l}_{N}}\left(\Phi_{s}^{\lambda_{1}}\left(z_{1}\right) \ldots \Phi_{s}^{\lambda_{n}}\left(z_{n}\right)\right)$ varies as one moves infinitesimally along a path in $\operatorname{Bun}_{S L(N)}$, as $\mathcal{P}$ is subjected to infinitesimal deformations. Consequently, we have an interpretation of a sheaf of coinvariants on $\operatorname{Bun}_{S L(N)}$, where the fibre of this sheaf over each point in $\operatorname{Bun}_{S L(N)}$ is just the space $H_{\mathfrak{s l}_{N}}\left(\Phi_{s}^{\lambda_{1}}\left(z_{1}\right) \ldots \Phi_{s}^{\lambda_{n}}\left(z_{n}\right)\right)$ of coinvariants corresponding to a particular bundle $\mathcal{P}$ that one can consistently define over $\Sigma$ using the affine $S L(N)$-algebra of the sigma-model on $X=S L(N) / B$. Note howeover, that since we are dealing with an 
affine $S L(N)$ algebra at the critical level $k=-h^{\vee}$, the dimension of the space of coinvariants will be different over different points in $\operatorname{Bun}_{S L(N)}$. In other words, the sheaf of coinvariants on $\operatorname{Bun}_{S L(N)}$ does not have a structure of a vector bundle, since the fibre space of a vector bundle must have a fixed dimension over different points on the base. Put abstractly, this is because $\widehat{\mathfrak{s l}}_{N}$-modules at the critical level may only be exponentiated to a subgroup of the Kac-Moody group $\widehat{S L(N)}$. Nevertheless, the sheaf of coinvariants is a twisted $\mathcal{D}$-module on $\operatorname{Bun}_{S L(N)}$ [6].

From the above discussion, one can also make the following physical observation. Notice that the variation $\left.\delta_{\eta}\left\langle\Phi_{s}^{\lambda_{1}}\left(z_{1}\right) \ldots \Phi_{s}^{\lambda_{n}}\left(z_{n}\right)\right)\right\rangle=\left\langle\oint_{C} d z \sum_{a} \eta_{a}(z) J^{a}(z) \Phi_{s}^{\lambda_{1}}\left(z_{1}\right) \ldots \Phi_{s}^{\lambda_{n}}\left(z_{n}\right)\right\rangle$ in the correlation function as one moves along $\operatorname{Bun}_{S L(N)}$, can be interpreted, at the lowest order in sigma-model perturation theory, as a variation in the correlation function due to a marginal deformation of the sigma-model action by the term $\oint d z \eta(z)$. Since a deformation of the action by the dimensionless term $\oint d z \eta(z)$ is tantamount to a displacement in the moduli space of the sigma-model itself, it will mean that $\left.\delta_{\eta}\left\langle\Phi_{s}^{\lambda_{1}}\left(z_{1}\right) \ldots \Phi_{s}^{\lambda_{n}}\left(z_{n}\right)\right)\right\rangle$ is also the change in the correlation function as one varies the moduli of the sigma-model. This implies that $\operatorname{Bun}_{S L(N)}$ will at least span a subspace of the entire moduli space of the sigma-model on $X=S L(N) / B$. This should come as no surprise since $\mathcal{P}$ is actually associated to the affine $S L(N)$-algebra of the sigma-model on $X=S L(N) / B$ as explained above, and moreover, the affine $S L(N)$ - algebra does depend on the moduli of the sigma-model as mentioned in $\S 2$ and $\S 3$.

Last but not least, note that the sheaf of coinvariants can also be obtained purely mathematically [6] via a localisation functor $\Delta$, which maps the set $V_{\chi}$ - consisting of all polynomials $F(\mathcal{J}(z)$ ) (which exist in the chiral algebra of the twisted sigma-model on $S L(N) / B$ ) that are defined over the field of complex numbers and the $c$-number operators $S^{\left(s_{i}\right)}(z)$, and that are of arbitrary positive degree in the quantum operator $\mathcal{J}(z)=$ $\frac{1}{\left(-n_{1}-1\right) ! \ldots\left(-n_{m}-1\right) !}: \partial_{z}^{-n_{1}-1} J^{a_{1}}(z) \ldots \partial_{z}^{-n_{m}-1} J^{a_{m}}(z):$ - to the corresponding sheaf $\Delta\left(V_{\chi}\right)$ of coinvaraints on $\operatorname{Bun}_{S L(N)}$, where $\chi$ denotes a parameterisation of $V_{\chi}$ that depends on the choice of the set of $S^{\left(s_{i}\right)}(z)$ fields for $i=1, \ldots, \operatorname{rank}\left(\mathfrak{s l}_{N}\right)$. In other words, the sheaf of coinvariants will be parameterised by $\chi 12$ This observation is pivotal in the mathematical description of the correspondence between Hecke eignesheaves on $\operatorname{Bun}_{S L(N)}$ and flat holomorphic ${ }^{L} S L(N)$-bundles on $\Sigma$ via the algebraic CFT approach to the geometric Langlands program [6]. As we will explain below, this parameterisation of the sheaf of coinvariants on

\footnotetext{
${ }^{12}$ Note that in order to be consistent with the notation used in the mathematical literature, we have chosen to use the symbol $\chi$ to label the parameterisation of $V_{\chi}$. Hopefully, $\chi$ that appears here and henceforth will not be confused with the one-dimensional representation $\chi$ of $\widehat{\mathfrak{g}}^{\prime}$ in $\S 3$.
} 
$\operatorname{Bun}_{S L(N)}$ by the set of $S^{s_{i}}(z)$ fields can be shown to arise physically in the sigma-model as well.

$A \mathfrak{z}\left(\widehat{\mathfrak{s l}}_{N}\right)$-Dependent Realisation of the Affine $S L(N)$ Algebra at the Critical Level

Before one can understand how, within the context of the sigma-model on $X=S L(N) / B$, the sheaf of coinvariants can be parameterised by a choice of the set of $S^{s_{i}}(z)$ fields for $i=1, \ldots, \operatorname{rank}\left(\mathfrak{s l}_{N}\right)$, it will be necessary for us to understand how one can achieve a $\mathfrak{z}\left(\widehat{\mathfrak{s l}}_{N}\right)$ dependent realisation of the affine $S L(N)$ OPE algebra at $k=-h^{\vee}$ spanned by the set of $J^{a}(z)$ currents that correspond to classes in $H^{0}\left(X, \mathcal{O}_{X}^{c h}\right)$, where $X=S L(N) / B$.

To this end, let us start with the case of the affine $S L(2)$ OPE algebra at level $k=-2$, spanned by the currents $\left\{J_{+}, J_{-}, J_{3}\right\}$ in the holomorphic chiral algebra of the twisted sigmamodel on $X=S L(2) / B$, that correspond to classes in $H^{0}\left(X, \mathcal{O}_{X}^{c h}\right)$. Recall that the set $\left\{J_{+}, J_{-}, J_{3}\right\}$ can be expressed in terms of the fields of the free $\beta \gamma$ system associated to the sheaf $\mathcal{O}_{X}^{c h}$ of CDO's on $X=S L(2) / B$ in (2.7), (2.8) and (2.10) respectively. As explained, these are classes in $H^{0}\left(X, \mathcal{O}_{X}^{c h}\right)$, i.e., if the set $\left\{J_{+}, J_{-}, J_{3}\right\}$ were to be defined on the North pole of $X=S L(2) / B \simeq \mathbb{P}^{1}$, while the set $\left\{\widetilde{J}_{+}, \widetilde{J}_{-}, \widetilde{J}_{3}\right\}$ were to defined their corresponding counterparts on the South pole of $X=S L(2) / B \cong \mathbb{P}^{1}$, one will have $\widetilde{J}_{+}-J_{+}=0, \widetilde{J}_{-}-J_{-}=0$ and $\widetilde{J}_{3}-J_{3}=0$.

Let us now modify the expressions of $\left\{J_{+}, J_{-}, J_{3}\right\}$ as follows:

$$
\begin{aligned}
J_{+}^{\prime}(z) & =-: \gamma^{2}(z) \beta(z):+2 \partial_{z} \gamma(z)+\frac{1}{2} \gamma(z) c(z), \\
J_{-}^{\prime}(z) & =\beta(z) \\
J_{3}^{\prime}(z) & =-: \gamma(z) \beta(z):+\frac{1}{2} c(z)
\end{aligned}
$$

where $c(z)$ is a classical $c$-number field that is holomorphic in $z$ and of conformal dimension one, i.e., it has a Laurent expansion given by $c(z)=\sum_{n \in \mathbb{Z}} c_{n} z^{-n-1}$. Since $c(z)$ is a classical field, it will not participate as an interacting quantum field in any of the OPE's amongst the quantum operators $\left\{J_{+}^{\prime}, J_{-}^{\prime}, J_{3}^{\prime}\right\}$. Rather, it will just act as a simple multiplication on the $\gamma(z)$ and $\beta(z)$ fields, or functions thereof. Moreover, this means that $c(z)$, like $S(z)$, must also be trivial in the $\bar{Q}_{+}$-cohomology of the twisted sigma-model on $S L(2) / B$ at the quantum level, i.e., it can be expressed as a $\bar{Q}_{+}$-exact term $\left\{\bar{Q}_{+}, \ldots\right\}$ in the quantum theory. Now, recall that we had the (non quantum-corrected) geometrical gluing relation $\gamma=1 / \widetilde{\gamma}$, where $\gamma$ and $\widetilde{\gamma}$ are defined on the North and South poles of $X=S L(2) / B \simeq \mathbb{P}^{1}$ respectively. This expression means that $\gamma$ defines a global section of the sheaf $\widehat{\mathcal{O}}_{X}^{c h}$. From our $\bar{Q}_{+}$-Cech cohomology dictionary, this will mean that $\gamma(z)$ must correspond to an operator in the 
twisted sigma-model on $X$ that is annihilated by the quantum action of $\bar{Q}_{+}$. This, together with the fact that $c(z)$ can be expressed as $\left\{\bar{Q}_{+}, \ldots\right\}$, will mean that the term $\frac{1}{2} \gamma(z) c(z)$ in $J_{+}^{\prime}(z)$ of (4.6) above, can be written as a $\bar{Q}_{+}$-exact term $\left\{\bar{Q}_{+}, \ldots\right\}$. Likewise, the term $\frac{1}{2} c(z)$ in $J_{3}^{\prime}(z)$ of (4.8) can also be written as a $\bar{Q}_{+}$-exact term $\left\{\bar{Q}_{+}, \ldots\right\}$. Consequently, since $\bar{Q}_{+}^{2}=0$ even at the quantum level, $\left\{J_{+}^{\prime}, J_{-}^{\prime}, J_{3}^{\prime}\right\}$ continues to be a set of quantum operators that are $\bar{Q}_{+}$-closed and non- $\bar{Q}_{+}$-exact, which therefore correspond to classes in $H^{0}\left(X, \widehat{\mathcal{O}}_{X}^{c h}\right)$. Since the OPE's of $\bar{Q}_{+}$-exact terms such as $\frac{1}{2} \gamma(z) c(z)$ and $\frac{1}{2} c(z)$ with the other $\bar{Q}_{+}$-closed terms $\left\{-\gamma^{2} \beta+2 \partial_{z} \gamma, \beta,-\gamma \beta\right\}$ that correspond respectively to the set of original operators $\left\{J_{+}, J_{-}, J_{3}\right\}$ must again result in $\bar{Q}_{+}$-exact terms that are trivial in $\bar{Q}_{+}{ }^{-}$ cohomology, they can be discarded in the OPE's involving the set of operators $\left\{J_{+}^{\prime}, J_{-}^{\prime}, J_{3}^{\prime}\right\}$, i.e., despite being expressed differently from the set of original operators $\left\{J_{+}, J_{-}, J_{3}\right\}$, the set of operators $\left\{J_{+}^{\prime}, J_{-}^{\prime}, J_{3}^{\prime}\right\}$ will persist to generate an affine $S L(2)$ OPE algebra at the critical level $k=-2$. In other words, via the set of modified operators $\left\{J_{+}^{\prime}, J_{-}^{\prime}, J_{3}^{\prime}\right\}$ and their corresponding Laurent modes, we have a different realisation of the affine $S L(2)$ algebra at the critical level $k=-2$.

Obviously, from (4.6)-(4.8), we see that the above realisation depends on the choice of $c(z)$. What determines $c(z)$ then? To answer this, let us first recall that the SegalSugawara tensor $S^{\prime}(z)$ in the context of the modified operators $\left\{J_{+}^{\prime}, J_{-}^{\prime}, J_{3}^{\prime}\right\}$, can be expressed as $S^{\prime}(z)=:\left(J_{+}^{\prime} J_{-}^{\prime}+J_{3}^{\prime 2}\right)(z)$ : in the quantum theory. However, recall also that the original Segal-Sugawara tensor given by $S(z)=:\left(J_{+} J_{-}+J_{3}^{2}\right)(z)$ : acts by zero in the quantum theory. This means that the non-vanishing contributions to $S^{\prime}(z)$ come only from terms that involve the $c(z)$ field. A simple computation will show that $S^{\prime}(z)=\frac{1}{4} c(z)^{2}-\frac{1}{2} \partial_{z} c(z)$. As required, $S^{\prime}(z)$ is a classical holomorphic field of dimension-two. Clearly, a unique choice of $S^{\prime}(z)$ will determine a unique $c(z)$. In summary, we can generate different realisations of the affine $S L(2)$ OPE algebra at level $k=-h^{\vee}$ via the set of operators $\left\{J_{+}^{\prime}, J_{-}^{\prime}, J_{3}^{\prime}\right\}$, that are parameterised by the choice of the corresponding Segal-Sugawara tensor $S^{\prime}(z)$ in the classical holomorphic chiral algebra of the purely bosonic sector of the twisted $(0,2)$ sigma-model on $X=S L(2) / B$. Since the Laurent modes of $S^{\prime}(z)$ span the centre $\mathfrak{z}\left(\widehat{\mathfrak{s l}}_{2}\right)$ of the completed universal enveloping algebra of $\widehat{\mathfrak{s l}}_{2}$ at the critical level $k=-2$, we effectively have a $\mathfrak{z}\left(\widehat{\mathfrak{s l}}_{2}\right)$-dependent realisation of the affine $S L(2)$ (OPE) algebra at the critical level.

The above arguments can actually be extended to any $S L(N)$, not just $S L(2)$, i.e., for a twisted sigma-model on $X=S L(N) / B$, one can always find different realisations of an affine $S L(N)$ OPE algebra at the critical level $k=-h^{\vee}$ that are spanned by the local operators in the holomorphic chiral algebra of the sigma-model which correspond to classes 
in $H^{0}\left(X, \widehat{\mathcal{O}}_{X}^{c h}\right)$, that are $\mathfrak{z}\left(\widehat{\mathfrak{s l}}_{N}\right)$-dependent. We shall now import some important results in [31] to demonstrate this. Firstly, consider the set of local operators composed out of the $N(N-1) / 2$ (i.e. $\left.\operatorname{dim}_{\mathbb{C}} X\right)$ free $\beta_{i}(z)$ and $\gamma^{i}(z)$ fields of the $N(N-1) / 2$ linear $\beta \gamma$ systems associated to the sheaf of CDO's on $X$ :

$$
\begin{aligned}
& J_{-}^{i}(z)=\beta^{\alpha_{i}}(z)+\sum_{\varphi \in \Delta_{+}}: P_{\varphi}^{i}\left(\gamma^{\alpha}(z)\right) \beta^{\varphi}(z): \\
& J_{c}^{k}(z)=-\sum_{\varphi \in \Delta_{+}} \varphi\left(h^{k}\right): \gamma^{\varphi}(z) \beta^{\varphi}(z): \\
& J_{+}^{i}(z)=\sum_{\varphi \in \Delta_{+}}: Q_{\varphi}^{i}\left(\gamma^{\alpha}(z)\right) \beta^{\varphi}(z):+c_{i} \partial_{z} \gamma^{\alpha_{i}}(z),
\end{aligned}
$$

where the subscripts $\{ \pm, c\}$ denote a Cartan decomposition of the Lie algebra $\mathfrak{s l}_{N}$ under which the $J(z)$ local operators can be classified (as in $\S 2$ ), the superscript $\alpha_{i}$ denotes the free field that can be identified with the $i^{\text {th }}$ positive root of $\mathfrak{s l}_{N}$ where $i=1, \ldots, N(N-1) / 2$, $h^{k}$ is an element of the Cartan subalgebra of $\mathfrak{s l}_{N}$ where $k=1, \ldots, N-1, \varphi\left(h^{k}\right)$ is the $k^{t h}$ component of the root $\varphi$, the symbol $\Delta_{+}$denotes the set of positive roots of $\mathfrak{s l}_{N}$, the $c_{i}$ 's are complex constants, and lastly, $P_{\varphi}^{i}, Q_{\varphi}^{i}$ are some polynomials in the $\gamma^{\alpha}$ free fields.

Theorem 4.3 of [31] tells us that the Laurent modes of the above set of local operators $\left\{J_{ \pm}^{i}, J_{c}^{k}\right\}$ generate an affine $S L(N)$ algebra at the critical level $k=-h^{\vee}$, i.e., the set $\left\{J_{ \pm}^{i}, J_{c}^{k}\right\}$ will span an affine $S L(N)$ OPE algebra at the critical level $k=-h^{\vee}$. In fact, for the case of $S L(2)$, we have the identification $J_{ \pm}^{i} \leftrightarrow J_{ \pm}$and $J_{c}^{k} \leftrightarrow J_{3}$, where $\left\{J_{+}, J_{-}, J_{3}\right\}$ is the set of local currents in the holomorphic chiral algebra of the twisted sigma-model on $S L(2) / B$ in (2.7), (2.8) and (2.10) which generates an affine $S L(2)$ OPE algebra at the critical level $k=-2$. The fact that the currents $\left\{J_{ \pm}^{i}, J_{c}^{k}\right\}$ are composed purely out of free $\beta_{i}$ and $\gamma^{i}$ fields, and the fact that there will always be classes in $H^{0}\left(X, \mathcal{O}_{X}^{c h}\right)$ which correspond to operators that generate an affine $S L(N)$ OPE algebra [11], will together mean that the set of currents $\left\{J_{ \pm}^{i}, J_{c}^{k}\right\}$ must correspond (up to $\bar{Q}_{+}$-exact terms at worst) to classes in $H^{0}\left(X, \mathcal{O}_{X}^{c h}\right)$, i.e., the global sections of the sheaf $\mathcal{O}_{X}^{c h}$ of CDO's on $X=S L(N) / B$. Equivalently, this means that the set of local current operators $\left\{J_{ \pm}^{i}, J_{c}^{k}\right\}$ will be $\bar{Q}_{+}$-closed and hence lie in the holomorphic chiral algebra of the twisted sigma-model on $X=S L(N) / B$.

Proceeding as we did for the $S L(2)$ case discussed above, let us now consider a modifi- 
cation $\left\{J_{ \pm}^{i^{\prime}}, J_{c}^{k^{\prime}}\right\}$ of the set of currents $\left\{J_{ \pm}^{i}, J_{c}^{k}\right\}$, where

$$
\begin{aligned}
& J_{-}^{i^{\prime}}(z)=\beta^{\alpha_{i}}(z)+\sum_{\varphi \in \Delta_{+}}: P_{\varphi}^{i}\left(\gamma^{\alpha}(z)\right) \beta^{\varphi}(z): \\
& J_{c}^{k^{\prime}}(z)=-\sum_{\varphi \in \Delta_{+}} \varphi\left(h^{k}\right): \gamma^{\varphi}(z) \beta^{\varphi}(z):+b^{i}(z), \\
& J_{+}^{i^{\prime}}(z)=\sum_{\varphi \in \Delta_{+}}: Q_{\varphi}^{i}\left(\gamma^{\alpha}(z)\right) \beta^{\varphi}(z):+c_{i} \partial_{z} \gamma^{\alpha_{i}}(z)+b^{i}(z) \gamma^{\alpha_{i}}(z),
\end{aligned}
$$

and the $b^{i}(z)$ 's are just classical $c$-number functions that are holomorphic in $z$ and of conformal dimension one - it can be Laurent expanded as $b^{i}(z)=\sum_{n \in \mathbb{Z}} b_{n}^{i} z^{-n-1} 13$ Since the $b^{i}(z)$ 's are classical fields, they will not participate as interacting quantum fields in any of the OPE's amongst the quantum operators $\left\{J_{+}^{i^{\prime}}, J_{-}^{i^{\prime}}, J_{3}^{k^{\prime}}\right\}$. Rather, they will just act as a simple multiplication on the $\gamma^{\alpha_{i}}(z)$ and $\beta^{\alpha_{i}}(z)$ fields, or functions thereof. Moreover, this means that the $b^{i}(z)^{\prime}$ s, must be trivial in the $\bar{Q}_{+}$-cohomology of the twisted sigma-model on $S L(N) / B$ at the quantum level, i.e., it can be expressed as a $\bar{Q}_{+}$-exact term $\left\{\bar{Q}_{+}, \ldots\right\}$ in the quantum theory. Now, recall that we had the (non quantum-corrected) geometrical gluing relation $\gamma^{\alpha_{i}}=g^{\alpha_{i}}\left(\gamma^{\alpha}\right)$, where each $\gamma^{\alpha_{i}}$ and $g^{\alpha_{i}}\left(\gamma^{\alpha}\right)$ is defined in the open set $U_{1}$ and $U_{2}$ respectively of the intersection $U_{1} \cap U_{2}$ in $X$. This expression means that the $\gamma^{\alpha_{i}}$ 's define global sections of the sheaf $\widehat{\mathcal{O}}_{X}^{c h}$. From our $\bar{Q}_{+}$-Cech cohomology dictionary, this will mean that each $\gamma^{\alpha_{i}}(z)$ must correspond to an operator in the twisted sigma-model on $X$ that is annihilated by $\bar{Q}_{+}$at the quantum level. This, together with the fact that $b^{i}(z)$ 's can be expressed as $\left\{\bar{Q}_{+}, \ldots\right\}$, will mean that the $b^{i}(z) \gamma^{\alpha_{i}}(z)$ term in $J_{+}^{i^{\prime}}(z)$ of (4.14) above, can be written as a $\bar{Q}_{+}$-exact term $\left\{\bar{Q}_{+}, \ldots\right\}$. Likewise, the $b^{i}(z)$ term in $J_{c}^{k^{\prime}}(z)$ of (4.13) can also be written as a $\bar{Q}_{+}$-exact term $\left\{\bar{Q}_{+}, \ldots\right\}$. Consequently, since $\bar{Q}_{+}^{2}=0$ even at the quantum level, $\left\{J_{+}^{i^{\prime}}, J_{-}^{i^{\prime}}, J_{3}^{i^{\prime}}\right\}$ continues to be a set of quantum operators that are $\bar{Q}_{+}$-closed and non- $\bar{Q}_{+}$-exact, which therefore correspond to classes in $H^{0}\left(X, \widehat{\mathcal{O}}_{X}^{c h}\right)$. Since the OPE's of $\bar{Q}_{+}$-exact terms such as $b^{i}(z) \gamma^{\alpha_{i}}(z)$ and $b^{i}(z)$ with the other $\bar{Q}_{+}$-closed terms such as $\left(\sum_{\varphi \in \Delta_{+}}: Q_{\varphi}^{i}\left(\gamma^{\alpha}\right) \beta^{\varphi}:+c_{i} \partial_{z} \gamma^{\alpha_{i}}\right),\left(\beta^{\alpha_{i}}+\sum_{\varphi \in \Delta_{+}}: P_{\varphi}^{i}\left(\gamma^{\alpha}\right) \beta^{\varphi}:\right)$, and $\left(-\sum_{\varphi \in \Delta_{+}} \varphi\left(h^{k}\right): \gamma^{\varphi} \beta^{\varphi}:\right)$ that correspond respectively to the set of original operators $J_{+}^{i}, J_{-}^{i}$, and $J_{c}^{k}$, must again result in $\bar{Q}_{+}$-exact terms that are trivial in $\bar{Q}_{+}$-cohomology, they can be discarded in the OPE's involving the set of operators $\left\{J_{+}^{i^{\prime}}, J_{-}^{i^{\prime}}, J_{3}^{i^{\prime}}\right\}$, i.e., despite being expressed differently

\footnotetext{
${ }^{13}$ Note that the explicit expression of $b(z)$ cannot be arbitrary. It has to be chosen appropriately to ensure that the Segal-Sugawara tensor and its higher spin analogs given by the $S^{\left(s_{i}\right)}(z)$ 's, can be identified with the space of ${ }^{L} \mathfrak{s l}_{N}$-opers on the formal disc $D$ in $\Sigma$ as necessitated by the isomorphism $\mathfrak{z}\left(\widehat{\mathfrak{s l}}_{N}\right) \simeq \mathcal{W}_{\infty}\left({ }^{L} \widehat{\mathfrak{s l}}_{N}\right)$ from the duality of classical $\mathcal{W}$-algebras for $G=S L(N)$. For example, the expression of $b(z)$ as $\frac{1}{2} c(z)$ in the $G=S L(2)$ case ensures that $S^{\prime}(z)=\frac{1}{4} c^{2}(z)-\frac{1}{2} \partial_{z} c(z)$ can be identified with a projective connection on $D$ for each choice of $c(z)$.
} 
from the set of original operators $\left\{J_{+}^{i}, J_{-}^{i}, J_{c}^{k}\right\}$, the set of operators $\left\{J_{+}^{i^{\prime}}, J_{-}^{i^{\prime}}, J_{c}^{i^{\prime}}\right\}$ will persist to generate an affine $S L(N)$ OPE algebra at the critical level $k=-h^{\vee}$. In other words, via the set of modified operators $\left\{J_{ \pm}^{i^{\prime}}, J_{c}^{k^{\prime}}\right\}$ and their corresponding Laurent modes, we have a different realisation of the affine $S L(N)$ algebra at the critical level $k=-h^{\vee}$. This is consistent with Theorem 4.7 of [31], which states that the set $\left\{J_{ \pm}^{i^{\prime}}, J_{c}^{k^{\prime}}\right\}$ of modified operators will persist to generate an affine $S L(N)$ OPE algebra at the critical level $k=-h^{\vee}$.

Obviously, from (4.12)-(4.14), we see that the above realisation depends on the choice of the $b^{i}(z)$ 's. What determines the $b^{i}(z)$ 's then? To answer this, let us first recall that the SegalSugawara tensor $S^{(2)^{\prime}}(z)$ and its higher spin analogs $S^{\left(s_{i}\right)^{\prime}}(z)$ in the context of the modified operators $\left\{J_{+}^{i^{\prime}}, J_{-}^{i^{\prime}}, J_{c}^{k^{\prime}}\right\} \in\left\{J^{a^{\prime}}\right\}$, can be expressed as $S^{\left(s_{i}\right)^{\prime}}(z)=\tilde{d}_{a_{1} a_{2} \ldots a_{s_{i}}}: J^{a_{1}^{\prime}} J^{a_{2}^{\prime}} \ldots J^{a_{s_{i}}^{\prime}}(z)$ : in the quantum theory. However, recall also that the original Segal-Sugawara tensor and its higher spin analogs, expressed as $S^{\left(s_{i}\right)}(z)=\tilde{d}_{a_{1} a_{2} \ldots a_{s_{i}}}: J^{a_{1}} J^{a_{2}} \ldots J^{a_{s_{i}}}(z)$ : in terms of the original operators $\left\{J_{+}^{i}, J_{-}^{i}, J_{c}^{k}\right\} \in\left\{J^{a}\right\}$, act by zero in the quantum theory. This means that the non-vanishing contributions to any of the $S^{\left(s_{i}\right)}(z)$ 's come only from terms that involve the additional $b^{i}(z)$ fields. In fact, it is true that the $S^{\left(s_{i}\right)^{\prime}}(z)$ 's also act by zero in the quantum theory at $k=-h^{\vee}$, since they are also defined via a Sugawara-type construction which results in their quantum definition being $S^{\left(s_{i}\right)^{\prime}}(z)=\left(k+h^{\vee}\right) T^{\left(s_{i}\right)^{\prime}}(z)$. In other words, the $S^{\left(s_{i}\right)^{\prime}}(z)$ 's must be classical $c$-number fields of spin $s_{i}$ that are holomorphic in $z$. This implies that the $S^{\left(s_{i}\right)^{\prime}}(z)^{\prime}$ 's will be expressed solely in terms of the $c$-number $b^{i}(z)$ fields. An explicit example of this general statement has already been discussed earlier in the case of $S L(2)$ - for the $S L(2)$ case, we have the identification $J_{+}^{i^{\prime}} \leftrightarrow J_{+}^{\prime}, J_{-}^{i^{\prime}} \leftrightarrow J_{-}^{\prime} J_{c}^{k^{\prime}} \leftrightarrow J_{3}^{\prime}$, $S^{(2)^{\prime}}(z) \leftrightarrow S^{\prime}(z), b^{i}(z) \leftrightarrow \frac{1}{2} c(z)$ and $S^{(2)^{\prime}}(z)=\frac{1}{4} c^{2}(z)-\frac{1}{2} \partial_{z} c(z)$, whereby the choice of $S^{(2)^{\prime}}(z)$ determines $c(z)$. Consequently, a choice of the set of $S^{\left(s_{i}\right)^{\prime}}(z)$ fields will determine the $b^{i}(z)$ fields. Lastly, note that the $S^{\left(s_{i}\right)^{\prime}}(z)$ fields lie in the classical holomorphic chiral algebra of the purely bosonic sector of the twisted sigma-model on $X=S L(N) / B$, and their Laurent modes span the centre $\mathfrak{z}\left(\widehat{\mathfrak{s l}}_{N}\right)$ of the completed universal enveloping algebra of $\widehat{\mathfrak{s l}}_{N}$ at the critical level $k=-h^{\vee}$. Hence, we effectively have a $\mathfrak{z}\left(\widehat{\mathfrak{s l}}_{N}\right)$-dependent realisation of the affine $S L(N)(\mathrm{OPE})$ algebra at the critical level as claimed.

\section{$A \mathfrak{z}\left(\widehat{\mathfrak{s l}}_{N}\right)$-Dependent Parameterisation of the Sheaf of Coinvariants on Bun $\left.\operatorname{BL}_{S L}\right)$}

Now that we have seen how one can obtain a $\mathfrak{z}\left(\widehat{\mathfrak{s l}}_{N}\right)$-dependent realisation of the affine $S L(N)$ (OPE) algebra at the critical level, we can proceed to explain how, within the context of the sigma-model on $X=S L(N) / B$, the sheaf of coinvariants on $\operatorname{Bun}_{S L(N)}$ can be parameterised by a choice of the fields $S^{s_{i}}(z)$ for $i=1, \ldots, \operatorname{rank}\left(\mathfrak{s l}_{N}\right)$.

To this end, notice that since the primary field operators $\Phi_{s}^{\lambda}(z)$ are defined via the OPE's 
with the $J^{a}(z)$ currents of the $\widehat{\mathfrak{s l}}_{N}$ algebra at the critical level in (4.1), a different realisation of the $J^{a}(z)$ currents will also result in a different realisation of the $\Phi_{s}^{\lambda}(z)$ 's. Consequently, we will have a $\mathfrak{z}\left(\widehat{\mathfrak{s l}}_{N}\right)$-dependent realisation of the primary field operators $\Phi_{s}^{\lambda}(z)$. This amounts to a $\mathfrak{z}\left(\widehat{\mathfrak{s l}}_{N}\right)$-dependent realisation of their $n$-point correlation functions $\left\langle\Phi_{s}^{\lambda_{1}}\left(z_{1}\right) \ldots \Phi_{s}^{\lambda_{n}}\left(z_{n}\right)\right\rangle$. Since the correlation functions can be associated to a (vector in the) space of coinvariants as explained earlier, one will consequently have a $\mathfrak{z}\left(\widehat{\mathfrak{s l}}_{N}\right)$-dependent realisation of the sheaf of coinvariants on $\operatorname{Bun}_{S L(N)}$ as well, i.e., the sheaf of coinvariants will be parameterised by a choice of the fields $S^{s_{i}}(z)$ for $i=1, \ldots, \operatorname{rank}\left(\mathfrak{s l}_{N}\right)$.

A Correspondence Between Hecke Eigensheaves on Bun BL(N) $_{\text {and Flat }}{ }^{L} S L(N)$-Bundles on $\Sigma$

Finally, we shall now demonstrate that the above observation about a $\mathfrak{\mathfrak { z }}\left(\widehat{\mathfrak{s l}}_{N}\right)$-dependent realisation of the sheaf of coinvariants on $\operatorname{Bun}_{S L(N)}$, and the duality of classical $\mathcal{W}$-algebras for $G=S L(N)$ as an isomorphism of Poisson algebras $\mathfrak{z}\left(\widehat{\mathfrak{s l}}_{N}\right) \simeq \mathcal{W}_{\infty}\left({ }^{L} \widehat{\mathfrak{s l}}_{N}\right)$, will result in a correspondence between Hecke eigensheaves on $\operatorname{Bun}_{S L(N)}$ and flat holomorphic ${ }^{L} S L(N)$ bundles on the worldsheet $\Sigma$.

Firstly, note that the classsical $\mathcal{W}$-algebra $\mathcal{W}_{\infty}\left({ }_{\mathfrak{s l}_{N}}\right)$ is isomorphic to Fun $\mathrm{Op}_{L_{\mathfrak{s l}_{N}}}\left(D^{\times}\right)$, the algebra of functions on the space of ${ }^{L} \mathfrak{s l}_{N^{-}}$opers on the punctured disc $D^{\times}$in $\Sigma$, where an $\mathfrak{s l}_{N^{-}}$oper on $\Sigma$ is an $n^{\text {th }}$ order differential operator acting from $\Omega^{-(n-1) / 2}$ to $\Omega^{(n+1) / 2}$ (where $\Omega$ is the canonical line bundle on $\Sigma$ ) whose principal symbol is equal to 1 and subprincipal symbol is equal to 0 [6]. Roughly speaking, it may be viewed as a (flat) connection on an ${ }^{L} S L(N)$-bundle on $\Sigma$. In turn, Fun $\mathrm{Op}_{L_{\mathfrak{s} l_{N}}}\left(D^{\times}\right)$is related to the algebra Fun $\mathrm{Op}_{L_{\mathfrak{s l}}}(D)$ of functions on the space of ${ }^{L} \mathfrak{s l}_{N}$-opers on the formal disc $D$ in $\Sigma$, via Fun $\mathrm{Op}_{L_{\mathfrak{s l}}}\left(D^{\times}\right) \simeq$ $\widetilde{U}\left(\right.$ Fun $\left.\mathrm{Op}_{L_{\mathfrak{s l}}}(D)\right)$, where $\widetilde{U}$ is a functor from the category of vertex algebras to the category of Poisson algebras [31]. Since from the duality of classical $\mathcal{W}$-algebras for $G=S L(N)$, we have an isomorphism of Poisson algebras $\mathfrak{z}\left(\widehat{\mathfrak{s l}}_{N}\right) \simeq \mathcal{W}_{\infty}\left({ }^{L} \widehat{\mathfrak{s l}}_{N}\right)$, it will mean that the $S^{\left(s_{i}\right)}(z)$ 's will correspond to the components of the (numeric) ${ }^{L} \mathfrak{s l}_{N^{-}}$oper on the formal disc $D$ in $\Sigma[6$.

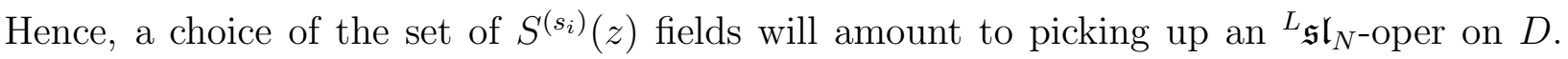
Since any ${ }^{L} \mathfrak{g}$-oper on $D$ can be extended to a regular ${ }^{L} \mathfrak{g}$-oper that is defined globally on $\Sigma$, it will mean that a choice of the set of $S^{\left(s_{i}\right)}(z)$ fields will determine a unique ${ }^{L} S L(N)$-bundle on $\Sigma$ (that admits a structure of an oper $\chi$ ) with a holomorphic connection.

Secondly, recall that we have a $\mathfrak{z}\left(\widehat{\mathfrak{s l}}_{N}\right)$-dependent realisation of the sheaf of coinvariants on $\operatorname{Bun}_{S L(N)}$ which depends on the choice of the fields $S^{s_{i}}(z)$ for $i=1, \ldots, \operatorname{rank}\left(\mathfrak{s l}_{N}\right)$. Hence, from the discussion in the previous paragraph, we see that we have a correspondence between a flat holomorphic ${ }^{L} S L(N)$-bundle on $\Sigma$ and a sheaf of coinvariants on $\operatorname{Bun}_{S L(N)}$. 
Lastly, recall that $\Delta\left(V_{\chi}\right)$ and therefore the sheaf of of coinvariants on $\operatorname{Bun}_{S L(N)}$ has a structure of a twisted $\mathcal{D}$-module on $\operatorname{Bun}_{S L(N)}$. The sought-after Hecke eigensheaf [6] is an untwisted holonomic $\mathcal{D}$-module $\Delta\left(V_{\chi}\right) \otimes K^{-1 / 2}$ on $\operatorname{Bun}_{S L(N)}$ with eigenvalue $E_{\chi}$, where $K$ is the canonical line bundle on $\operatorname{Bun}_{S L(N)}$, and $E_{\chi}$ is the unique ${ }^{L} S L(N)$-bundle corresponding to a particular choice of the set of $S^{\left(s_{i}\right)}(z)$ fields. In total, since tensoring with a globallydefined $K$ on $\operatorname{Bun}_{S L(N)}$ just maps, in a one-to-one fashion, $\Delta\left(V_{\chi}\right)$ to $\Delta\left(V_{\chi}\right) \otimes K^{-1 / 2}$, we find that we have a one-to-one correspondence between a Hecke eigensheaf on $\operatorname{Bun}_{S L(N)}$ and a flat holomorphic ${ }^{L} S L(N)$-bundle on $\Sigma$, where ${ }^{L} S L(N)=P S L(N)$, i.e., we have a geometric Langlands correspondence for $G=S L(N) 14$

\section{Physical Interpretation of the Hecke Eigensheaves on Bun $S L(N)$}

From all of our above results, we see that one can physically interpret the Hecke eigensheaf as follows. A local section of the fibre of the Hecke eigensheaf over a point $p$ in $\operatorname{Bun}_{S L(N)}$, will determine, for some holomorphic $S L(N)$-bundle on $\Sigma$ that corresponds to the point $p$ in the moduli space $\operatorname{Bun}_{S L(N)}$ of all holomorphic $S L(N)$-bundles on $\Sigma$, the value of any $n$-point correlation function $\left\langle\Phi_{s}^{\lambda_{1}}\left(z_{1}\right) \ldots \Phi_{s}^{\lambda_{n}}\left(z_{n}\right)\right\rangle$ of local bosonic operators in the holomorphic chiral algebra of the twisted $(0,2)$ sigma-model on $S L(N) / B$. And the geometric Langlands correspondence for our case of $G=S L(N)$ just tells us that for every flat, holomorphic $P S L(N)$-bundle that can be constructed over $\Sigma$, we have a unique way of characterising how an $n$-point correlation function of local bosonic primary operators in the holomorphic chiral algebra of a quasi-topological sigma-model with no boundaries like the twisted $(0,2)$ sigma-model on $S L(N) / B$, will vary under the local $S L(N)$-transformations generated by the affine $J^{a}(z)$ currents on the worldsheet described earlier.

\subsection{Hecke Operators and the Correlation Functions of Local Operators}

Consider the quantum operator $\mathcal{J}(z)=\frac{1}{\left(-n_{1}-1\right) ! \ldots\left(-n_{m}-1\right) !}: \partial_{z}^{-n_{1}-1} J^{a_{1}}(z) \ldots \partial_{z}^{-n_{m}-1} J^{a_{m}}(z):$. Note that since the $J^{a}(z)$ 's are $\bar{Q}_{+}$-closed and in the $\bar{Q}_{+}$-cohomology or holomorphic chiral algebra of the sigma-model on $S L(N) / B$, so will $\mathcal{J}(z)$ or polynomials $F(\mathcal{J}(z))$ of arbitrary

\footnotetext{
${ }^{14}$ Note that the above-mentioned flat holomorphic ${ }^{L} S L(N)$-bundles on $\Sigma$ are restricted to those that have a structure of an ${ }^{L} \mathfrak{g}$-oper on $\Sigma$. The space of connections of any such bundle only form a half-dimensional subspace in the moduli stack $\operatorname{Loc}_{L} G$ of the space of all connections on a particular flat ${ }^{L} G$-bundle, where $G=S L(N)$. Thus, our construction establishes the geometric Langlands correspondence only partially. However, it turns out that our construction can be generalised to include all flat ${ }^{L} G$-bundles on $\Sigma$ by considering in the correlation functions more general chiral operators that are labelled by finite-dimensional representations of $\mathfrak{g}$, which, in mathematical terms, is equivalent to making manifest the singular oper structure of any flat ${ }^{L} G$-bundle on $\Sigma[\underline{6}$.
} 
positive degree in $\mathcal{J}(z)$ (modulo polynomials of arbitrary positive degree in the $S^{\left(s_{i}\right)}(z)$ operators which necessarily act by zero and hence vanish in the quantum theory) 15

The set of local operators described by $F(\mathcal{J}(z))$ can be identified with the mathematically defined chiral vertex algebra $V_{-h^{\vee}}(\mathfrak{g})$ associated to $\widehat{\mathfrak{g}}$ at the critical level $k=-h^{\vee}$, where $\mathfrak{g}=\mathfrak{s l}_{N}$ in our present case. The action of the Hecke operator on a Hecke eigensheaf as defined in the axiomatic CFT sense, is equivalent to an insertion of an operator that lies in the chiral vertex algebra given by $m$ copies of $V_{-h^{\vee}}(\mathfrak{g})$, i.e., $\oplus_{m} V_{-h^{\vee}}(\mathfrak{g})$ [6]. Such an operator is again a polynomial operator of the form $F(\mathcal{J}(z))$. In short, the action of the Hecke operator is equivalent to inserting into the correlation functions of local primary field operators of the twisted $(0,2)$ sigma-model on $S L(N) / B$, other local operators that also lie in the holomorphic chiral algebra of the twisted $(0,2)$ sigma-model on $S L(N) / B$, which, as emphasised earlier, is a quasi-topological sigma-model with no boundaries. This is to be contrasted with the description of the Hecke operators (and Hecke eigensheaves) in the gauge-theoretic approach to the geometric Langlands program, where they are interpreted as 't Hooft line operators (and D-branes) in a topological sigma-model with boundaries. Our results therefore provide an alternative physical interpretation of these abstract objects of the geometric Langlands correspondence for $G=S L(N)$, to that furnished in the gaugetheoretic approach by Kapustin and Witten in [1].

\section{A The Twisted $(0,2)$ Sigma-Model and Sheaves of CDO's}

We shall review the relevant features of the twisted $(0,2)$ sigma-model considered by Witten in [10] and its relation to the theory of CDO's constructed by Malikov et al. in [11]. In our aim to keep this paper self-contained, we will present the relevant details in as comprehensive and coherent a manner as possible. The interested reader is encouraged to seek the original references for other details not covered in this appendix.

\section{A.1. The Sigma-Model with $(0,2)$ Supersymmetry}

Let us first recall the two-dimensional non-linear sigma-model with $(0,2)$ supersymmetry on a complex manifold $X$. It governs maps $\Phi: \Sigma \rightarrow X$, with $\Sigma$ being the worldsheet Riemann surface. By picking local coordinates $z, \bar{z}$ on $\Sigma$, and $\phi^{i}, \phi^{\bar{i}}$ on $X$, the map $\Phi$ can

\footnotetext{
${ }^{15}$ In order to show this, first note that $\partial_{z} J^{a}(z)=\left[L_{-1}, J^{a}(z)\right]$, where $L_{-1}=\oint d z T_{z z}(z)$. Since $\left[\bar{Q}_{+}, J^{a}(z)\right]=0$ even at the quantum level, it will mean that $\left[\bar{Q}_{+}, \partial_{z} J^{a}(z)\right]=\left[\left[\bar{Q}_{+}, L_{-1}\right], J^{a}(z)\right]=$ $\oint d z^{\prime}\left[\left[\bar{Q}_{+}, T_{z z}\left(z^{\prime}\right)\right], J^{a}(z)\right]=\oint d z^{\prime}\left[\partial_{z^{\prime}}\left(R_{i \bar{j}} \partial_{z^{\prime}} \phi^{i} \psi^{\bar{j}}\right), J^{a}(z)\right]=0$. One can proceed to repeat this argument and show that $\left[\bar{Q}_{+}, \partial_{z}^{m} J^{a}(z)\right]=0$ for any $m \geq 1$ at the quantum level always.
} 
then be described locally via the functions $\phi^{i}(z, \bar{z})$ and $\phi^{\bar{i}}(z, \bar{z})$. Let $\bar{K}$ be the anti-canonical bundle of $\Sigma$ (the bundle of one-forms of type $(0,1)$ ), whereby the right-moving spinor bundle of $\Sigma$ is given $\bar{K}^{1 / 2}$. Let $T X$ and $\overline{T X}$ be the holomorphic and anti-holomorphic tangent bundle of $X$. The right-moving fermi fields consist of $\psi^{i}$ and $\psi^{\bar{i}}$, which are smooth sections of the bundles $\bar{K}^{1 / 2} \otimes \Phi^{*} T X$ and $\bar{K}^{1 / 2} \otimes \Phi^{*} \overline{T X}$ respectively. Here, $\psi^{i}$ and $\psi^{\bar{i}}$ are superpartners of the scalar fields $\phi^{i}$ and $\phi^{\bar{i}}$. Let $g$ be the hermitian metric on $X$. The action is then given by

$$
S=\int_{\Sigma}\left|d^{2} z\right| \frac{1}{2} g_{i \bar{j}}\left(\partial_{z} \phi^{i} \partial_{\bar{z}} \phi^{\bar{j}}+\partial_{\bar{z}} \phi^{i} \partial_{z} \phi^{\bar{j}}\right)+g_{i \bar{j}} \psi^{i} D_{z} \psi^{\bar{j}}
$$

whereby $i, \bar{i}=1 \ldots, n=\operatorname{dim}_{\mathbb{C}} X,\left|d^{2} z\right|=i d z \wedge d \bar{z}$. In addition, $D_{z}$ is the $\partial$ operator on $\bar{K}^{1 / 2} \otimes \phi^{*} \overline{T X}$ using the pull-back of the Levi-Civita connection on $T X$. In formulas (using a local trivialisation of $\bar{K}^{1 / 2}$ ), we have 16

$$
D_{z} \psi^{\bar{j}}=\partial_{z} \psi^{\bar{j}}+\Gamma_{\overline{l k}}^{\bar{j}} \partial_{z} \phi^{\bar{l}} \psi^{\bar{k}}
$$

where $\Gamma_{\overline{l k}}^{\bar{j}}$ is the affine connection of $X$.

The infinitesimal transformation of the fields generated by the supercharge $\bar{Q}_{+}$under the first right-moving supersymmetry, is given by

$$
\begin{aligned}
\delta \phi^{i}=0, & \delta \phi^{\bar{i}}=\bar{\epsilon}_{-} \psi^{\bar{i}}, \\
\delta \psi^{\bar{i}}=0, & \delta \psi^{i}=-\bar{\epsilon}_{-} \partial_{\bar{z}} \phi^{i},
\end{aligned}
$$

while the infinitesimal transformation of the fields generated by the supercharge $Q_{+}$under the second right-moving supersymmetry, is given by

$$
\begin{array}{cl}
\delta \phi^{i}=\epsilon_{-} \psi^{i}, & \delta \psi^{\bar{i}}=-\epsilon_{-} \partial_{\bar{z}} \phi^{\bar{i}}, \\
\delta \psi^{i}=0, & \delta \phi^{\bar{i}}=0 .
\end{array}
$$

where $\left(\bar{\epsilon}_{-}\right) \epsilon_{-}$are (anti-)holomorphic sections of $\bar{K}^{-1 / 2}$.

\section{A.2. Twisting the Model}

Classically, the action (A.1) and therefore the model that it describes, possesses a rightmoving R-symmetry, giving rise to a $U(1)_{R}$ global symmetry group. Denoting $q_{R}$ to be the

\footnotetext{
${ }^{16}$ Note that we have used a flat metric and hence vanishing spin connection on the Riemann surface $\Sigma$ in writing these formulas.
} 
charge of the right-moving fermi fields under this symmetry group, we find that $\psi^{\bar{i}}$ and $\psi^{i}$ will have charge $q_{R}= \pm 1$ respectively. Quantum mechanically however, this symmetry is anomalous because of non-perturbative worldsheet instantons; the charge violations for the right-moving global symmetry is given by $\Delta q_{R}=\int_{\Sigma} \Phi^{*} c_{1}(T X)$.

In order to define a twisted variant of the model, the spins of the fermi fields need to be shifted by a multiple of their corresponding right-moving charge $q_{R}$ under the global $U(1)_{R}$ symmetry group; by considering a shift in the spin $S$ via $S \rightarrow S+\frac{1}{2}\left[(2 \bar{s}-1) q_{R}\right]$ (where $\bar{s}$ is a real number), the fermi fields of the twisted model will transform as smooth sections of the following bundles:

$$
\psi^{i} \in \Gamma\left(\bar{K}^{(1-\bar{s})} \otimes \Phi^{*} T X\right), \quad \psi^{\bar{i}} \in \Gamma\left(\bar{K}^{\bar{s}} \otimes \Phi^{*} \overline{T X}\right) .
$$

Notice that for $s=\bar{s}=\frac{1}{2}$, the fermi fields transform as smooth sections of the same tensored bundles defining the original $(0,2)$ sigma-model, i.e., we get back the untwisted model.

To make contact with the theory of CDO's, we shall consider the case where $\bar{s}=0$. Then, the fermi fields of the twisted model will transform as smooth sections of the following bundles:

$$
\psi_{\bar{z}}^{i} \in \Gamma\left(\bar{K}^{1} \otimes \Phi^{*} T X\right), \quad \psi^{\bar{i}} \in \Gamma\left(\Phi^{*} \overline{T X}\right)
$$

Notice that we have included additional indices in the above fields so as to reflect their new geometrical characteristics on $\Sigma$; the fermi field without a $\bar{z}$ index transform as a worldsheet scalar, while the fermi field with a $\bar{z}$ index transform as a $(0,1)$-form on the worldsheet. In addition, as reflected by the $i$, and $\bar{i}$ indices, all fields continue to be valued in the pull-back of the corresponding bundles on $X$. Thus, the action of the twisted variant of the $(0,2)$ sigma-model is given by

$$
S_{\mathrm{twist}}=\int_{\Sigma}\left|d^{2} z\right| \frac{1}{2} g_{i \bar{j}}\left(\partial_{z} \phi^{i} \partial_{\bar{z}} \phi^{\bar{j}}+\partial_{\bar{z}} \phi^{i} \partial_{z} \phi^{\bar{j}}\right)+g_{i \bar{j}} \psi_{\bar{z}}^{i} D_{z} \psi^{\bar{j}}
$$

A twisted theory is the same as an untwisted one when defined on a $\Sigma$ which is flat. Hence, locally (where one has the liberty to select a flat metric), the twisting does nothing at all. However, what happens non-locally may be non-trivial. In particular, note that globally, the supersymmetry parameters $\epsilon_{-}$and $\bar{\epsilon}_{-}$must now be interpreted as sections of different line bundles; in the twisted model, the transformation laws given by (A.3) and (A.4) are still valid, and because of the shift in the spins of the various fields, we find that for the laws to remain physically consistent, $\bar{\epsilon}_{-}$must now be a function on $\Sigma$ while $\epsilon_{-}$ must be a section of the non-trivial bundle $\bar{K}^{-1}$. One can therefore canonically pick $\bar{\epsilon}_{-}$ 
to be a constant and $\epsilon_{-}$to vanish, i.e., the twisted variant of the $(0,2)$ sigma-model has just one canonical global fermionic symmetry generated by the supercharge $\bar{Q}_{+}$. Hence, the infinitesimal transformation of the (twisted) fields under this single canonical symmetry must read (after setting $\bar{\epsilon}_{-}$to 1 )

$$
\begin{aligned}
& \delta \phi^{i}=0, \quad \delta \phi^{\bar{i}}=\psi^{\bar{i}}, \\
& \delta \psi^{\bar{i}}=0, \quad \delta \psi_{\bar{z}}^{i}=-\partial_{\bar{z}} \phi^{i} .
\end{aligned}
$$

From the $(0,2)$ supersymmetry algebra, we have $\bar{Q}_{+}^{2}=0$. In addition, (after twisting) $\bar{Q}_{+}$ transforms as a scalar. Consequently, we find that the symmetry is nilpotent i.e., $\delta^{2}=0$ (off-shell), and behaves as a BRST-like symmetry.

Note at this point that the transformation laws of (A.8) can be expressed in terms of the BRST operator $\bar{Q}_{+}$, whereby $\delta W=\left\{\bar{Q}_{+}, W\right\}$ for any field $W$. One can then show that the action (A.7) can be written as

$$
S_{\mathrm{twist}}=\int_{\Sigma}\left|d^{2} z\right|\left\{\bar{Q}_{+}, V\right\}+S_{\mathrm{top}}
$$

where

$$
V=-g_{i \bar{j}} \psi_{\bar{z}}^{i} \partial_{z} \phi^{\bar{j}}
$$

while

$$
S_{\mathrm{top}}=\frac{1}{2} \int_{\Sigma} g_{i \bar{j}}\left(\partial_{z} \phi^{i} \partial_{\bar{z}} \phi^{\bar{j}}-\partial_{\bar{z}} \phi^{i} \partial_{z} \phi^{\bar{j}}\right)
$$

is $\int_{\Sigma} \Phi^{*}(K)$, the integral of the pull-back to $\Sigma$ of the $(1,1)$-form $K=\frac{i}{2} g_{i \bar{j}} d \phi^{i} \wedge d \phi^{\bar{j}}$.

Notice that since $\bar{Q}_{+}^{2}=0$, the first term on the RHS of (A.9) is invariant under the transformation generated by $\bar{Q}_{+}$. In addition, as mentioned in the introduction, we will be studying the twisted model in perturbation theory, where one does an expansion in the inverse of the large-radius limit. Hence, only the degree-zero maps of the term $\int_{\Sigma} \Phi^{*}(K)$ contribute to the path integral factor $e^{-S_{\text {twist }}}$. Therefore, in the perturbative limit, one can set $\int_{\Sigma} \Phi^{*}(K)=0$, i.e., $S_{\text {top }}$ can be set to zero. Thus, the action given in (A.9) is invariant under the BRST symmetry as required. Moreover, for the transformation laws of (A.8) to be physically consistent, $\bar{Q}_{+}$must have charge $q_{R}=1$ under the global $U(1)_{R}$ gauge group. Since $V$ has a corresponding charge of $q_{R}=-1, S_{\text {twist }}$ in (A.9) continues to be invariant under the $U(1)_{R}$ symmetry group at the classical level. In summary, the effective action in perturbation theory reads

$$
S_{\text {pert }}=\int_{\Sigma}\left|d^{2} z\right| g_{i \bar{j}}\left(\partial_{z} \phi^{\bar{j}} \partial_{\bar{z}} \phi^{i}+\psi_{\bar{z}}^{i} D_{z} \psi^{\bar{j}}\right),
$$


where it can also written as

$$
S_{\text {pert }}=\int_{\Sigma}\left|d^{2} z\right|\left\{\bar{Q}_{+}, V\right\} .
$$

Note that the original symmetries of the theory persist despite limiting ourselves to perturbation theory; even though $S_{\text {top }}=0$, from (A.13), one finds that $S_{\text {pert }}$ is invariant under the nilpotent BRST symmetry generated by $\bar{Q}_{+}$. It is also invariant under the $U(1)_{R}$ global symmetry. $S_{\text {pert }}$ shall henceforth be the action of interest in all our subsequent discussions.

\section{A.3. Chiral Algebras from the Twisted Sigma-Model}

\section{The Chiral Algebra}

Classically, the model is conformally invariant. The trace of the stress tensor from $S_{\text {pert }}$ vanishes, i.e., $T_{z \bar{z}}=0$. The other non-zero components of the stress tensor, at the classical level, are given by

$$
T_{z z}=g_{i \bar{j}} \partial_{z} \phi^{i} \partial_{z} \phi^{\bar{j}}
$$

and

$$
T_{\bar{z} \bar{z}}=g_{i \bar{j}} \partial_{\bar{z}} \phi^{i} \partial_{\bar{z}} \phi^{\bar{j}}+g_{i \bar{j}} \psi_{\bar{z}}^{i}\left(\partial_{\bar{z}} \psi^{\bar{j}}+\Gamma_{\overline{l k}}^{\bar{j}} \partial_{\bar{z}} \phi^{\bar{l}} \psi^{\bar{k}}\right) .
$$

Furthermore, one can go on to show that

$$
T_{\bar{z} \bar{z}}=\left\{\bar{Q}_{+},-g_{i \bar{j}} \psi_{\bar{z}}^{i} \partial_{\bar{z}} \phi^{\bar{j}}\right\}
$$

and

$$
\begin{aligned}
{\left[\bar{Q}_{+}, T_{z z}\right] } & =g_{i j} \partial_{z} \phi^{i} D_{z} \psi^{\bar{j}} \\
& =0 \text { (on-shell). }
\end{aligned}
$$

From (A.17) and (A.16), we see that all components of the stress tensor are $\bar{Q}_{+}$-invariant; $T_{z z}$ is an operator in the $\bar{Q}_{+}$-cohomology while $T_{\bar{z} \bar{z}}$ is $\bar{Q}_{+}$-exact and thus trivial in $\bar{Q}_{+^{-}}$ cohomology. The fact that $T_{z z}$ is not $\bar{Q}_{+}$-exact even at the classical level implies that the twisted model is not a two-dimensional topological field theory; rather, it is a twodimensional conformal field theory. This because the original model has $(0,2)$ and not $(2,2)$ supersymmetry. On the other hand, the fact that $T_{\bar{z} \bar{z}}$ is $\bar{Q}_{+}$-exact has some non-trivial consequences on the nature of the local operators in the $\bar{Q}_{+}$-cohomology. Let us discuss this further. 
We say that a local operator $\mathcal{O}$ inserted at the origin has dimension $(n, m)$ if under a rescaling $z \rightarrow \lambda z, \bar{z} \rightarrow \bar{\lambda} z$ (which is a conformal symmetry of the classical theory), it transforms as $\partial^{n+m} / \partial z^{n} \partial \bar{z}^{m}$, that is, as $\lambda^{-n} \bar{\lambda}^{-m}$. Classical local operators have dimensions $(n, m)$ where $n$ and $m$ are non-negative integers 17 However, only local operators with $m=0$ survive in $\bar{Q}_{+}$-cohomology. The reason for the last statement is that the rescaling of $\bar{z}$ is generated by $\bar{L}_{0}=\oint d \bar{z} \bar{z} T_{\bar{z} \bar{z}}$. As we noted in the previous paragraph, $T_{\bar{z} \bar{z}}$ is of the form $\left\{\bar{Q}_{+}, \ldots\right\}$, so $\bar{L}_{0}=\left\{\bar{Q}_{+}, V_{0}\right\}$ for some $V_{0}$. If $\mathcal{O}$ is to be admissible as a local physical operator, it must at least be true that $\left\{\bar{Q}_{+}, \mathcal{O}\right\}=0$. Consequently, $\left[\bar{L}_{0}, \mathcal{O}\right]=\left\{\bar{Q}_{+},\left\{V_{0}, \mathcal{O}\right\}\right\}$. Since the eigenvalue of $\bar{L}_{0}$ on $\mathcal{O}$ is $m$, we have $\left[\bar{L}_{0}, \mathcal{O}\right]=m \mathcal{O}$. Therefore, if $m \neq 0$, it follows that $\mathcal{O}$ is $\bar{Q}_{+}$-exact and thus trivial in $\bar{Q}_{+}$-cohomology.

By a similar argument, we can show that $\mathcal{O}$, as an element of the $\bar{Q}_{+}$-cohomology, varies holomorphically with $z$. Indeed, since the momentum operator (which acts on $\mathcal{O}$ as $\left.\partial_{\bar{z}}\right)$ is given by $\bar{L}_{-1}$, the term $\partial_{\bar{z}} \mathcal{O}$ will be given by the commutator $\left[\bar{L}_{-1}, \mathcal{O}\right]$. Since $\bar{L}_{-1}=\oint d \bar{z} T_{\bar{z} \bar{z}}$, we will have $\bar{L}_{-1}=\left\{\bar{Q}_{+}, V_{-1}\right\}$ for some $V_{-1}$. Hence, because $\mathcal{O}$ is physical such that $\left\{\bar{Q}_{+}, \mathcal{O}\right\}=0$, it will be true that $\partial_{\bar{z}} \mathcal{O}=\left\{\bar{Q}_{+},\left\{V_{-1}, \mathcal{O}\right\}\right\}$ and thus vanishes in $\bar{Q}_{+}$-cohomology.

The observations that we have so far are based solely on classical grounds. The question that one might then ask is whether these observations will continue to hold when we eventually consider the quantum theory. The key point to note is that if it is true classically that a cohomology vanishes, it should continue to do so in perturbation theory, when quantum effects are small enough. Since the above observations were made based on the classical fact that $T_{\bar{z} \bar{z}}$ vanishes in $\bar{Q}_{+}$-cohomology, they will continue to hold at the quantum level. Let us look at the quantum theory more closely.

\section{The Quantum Theory}

Quantum mechanically, the conformal structure of the theory is violated by a non-zero one-loop $\beta$-function; renormalisation adds to the classical action $S_{\text {pert }}$ a term of the form:

$$
\Delta_{1-l o o p}=c_{1} R_{i \bar{j}} \partial_{z} \phi^{\bar{j}} \psi_{\bar{z}}^{i}
$$

for some divergent constants $c_{1}$, where $R_{i \bar{j}}$ is the Ricci tensor of $X$. In the Calabi-Yau case, one can choose a Ricci-flat metric such that $\Delta_{1-\text { loop }}$ vanishes and the original action is restored. In this case, the classical observations made above continue to hold true. On

\footnotetext{
${ }^{17}$ Anomalous dimensions under RG flow may shift the values of $n$ and $m$ quantum mechanically, but the spin given by $(n-m)$, being an intrinsic property, remains unchanged.
} 
the other hand, in the "massive models" where $c_{1}(X) \neq 0$, there is no way to set $\Delta_{1-\text { loop }}$ to zero. Conformal invariance is necessarily lost, and there $i s$ nontrivial RG running. However, one can continue to express $T_{\bar{z} \bar{z}}$ as $\left\{\bar{Q}_{+}, \ldots\right\}$, i.e., it remains $\bar{Q}_{+}$-exact, and thus continues to vanish in $\bar{Q}_{+}$-cohomology. Hence, the above observations about the holomorphic nature of the local operators having dimension $(n, 0)$ continue to hold in the quantum theory.

We would also like to bring to the reader's attention another important feature of the $\bar{Q}_{+}$-cohomology at the quantum level. Recall that classically, we had $\left[\bar{Q}_{+}, T_{z z}\right]=0$ via the classical equations of motion. Notice that the classical expression for $T_{z z}$ is not modified at the quantum level (at least up to one-loop), since even in the non-Calabi-Yau case, the additional term of $\Delta_{1-\text { loop }}$ in the quantum action does not contribute to $T_{z z}$. However, due to one-loop corrections to the action of $\bar{Q}_{+}$, we have, at the quantum level

$$
\left[\bar{Q}_{+}, T_{z z}\right]=\partial_{z}\left(R_{i \bar{j}} \partial_{z} \phi^{i} \psi^{\bar{j}}\right)
$$

Note that the term on the RHS of (A.19) cannot be eliminated through the equations of motion in the quantum theory. Neither can we modify $T_{z z}$ (by subtracting a total derivative term) such that it continues to be $\bar{Q}_{+}$-invariant. This implies that in a 'massive' model, operators do not remain in the $\bar{Q}_{+}$-cohomology after general holomorphic coordinate transformations on the worldsheet, i.e., the model is not conformal at the level of the $\bar{Q}_{+}$-cohomology 18 However, $T_{z z}$ continues to be holomorphic in $z$ up to $\bar{Q}_{+}$-trivial terms; from the conservation of the stress tensor, we have $\partial_{\bar{z}} T_{z z}=-\partial_{z} T_{z \bar{z}}$, and $T_{z \bar{z}}$, while no longer zero, is now given by $T_{z \bar{z}}=\left\{\bar{Q}_{+}, G_{z \bar{z}}\right\}$ for some $G_{z \bar{z}}$, i.e., $\partial_{z} T_{z \bar{z}}$ continues to be $\bar{Q}_{+}$-exact, and $\partial_{\bar{z}} T_{z z} \sim 0$ in $\bar{Q}_{+}$-cohomology. The holomorphy of $T_{z z}$, together with the relation (A.19), has further implications for the $\bar{Q}_{+}$-cohomology of local operators; by a Laurent expansion of $T_{z z}$, 19 one can use $(\underline{\mathrm{A} .19})$ to show that $\left[\bar{Q}_{+}, L_{-1}\right]=0$. This means that operators remain in the $\bar{Q}_{+}$-cohomology after global translations on the worldsheet. In addition, recall that $\bar{Q}_{+}$is a scalar with spin zero in the twisted model. As shown few paragraphs before, we have the condition $\bar{L}_{0}=0$. Let the spin be $S$, where $S=L_{0}-\bar{L}_{0}$. Therefore, $\left[\bar{Q}_{+}, S\right]=0$ implies that $\left[\bar{Q}_{+}, L_{0}\right]=0$. In other words, operators remain in the $\bar{Q}_{+}$-cohomology after global dilatations of the worldsheet coordinates.

One can also make the following observations about the correlation functions of these local operators. Firstly, note that $\left\langle\left\{\bar{Q}_{+}, W\right\}\right\rangle=0$ for any $W$, and recall that for any lo-

\footnotetext{
${ }^{18}$ In $\S 2$ and $\S 3$, we will examine more closely, from a different point of view, the one-loop correction to the action of $\bar{Q}_{+}$associated with the beta-function, where (A.19) will appear in a different guise.

${ }^{19}$ Since we are working modulo $\bar{Q}_{+}$-trivial operators, it suffices for $T_{z z}$ to be holomorphic up to $\bar{Q}_{+}$-trivial terms before an expansion in terms Laurent coefficients is permitted.
} 
cal physical operator $\mathcal{O}_{\alpha}$, we have $\left\{\bar{Q}_{+}, \mathcal{O}_{\alpha}\right\}=0$. Since the $\partial_{\bar{z}}$ operator on $\Sigma$ is given by $\bar{L}_{-1}=\oint d \bar{z} T_{\bar{z} \bar{z}}$, where $T_{\bar{z} \bar{z}}=\left\{\bar{Q}_{+}, \ldots\right\}$, we find that $\partial_{\bar{z}}\left\langle\mathcal{O}_{1}\left(z_{1}\right) \mathcal{O}_{2}\left(z_{2}\right) \ldots \mathcal{O}_{s}\left(z_{s}\right)\right\rangle$ is given by $\oint d \bar{z}\left\langle\left\{\bar{Q}_{+}, \ldots\right\} \mathcal{O}_{1}\left(z_{1}\right) \mathcal{O}_{2}\left(z_{2}\right) \ldots \mathcal{O}_{s}\left(z_{s}\right)\right\rangle=\oint d \bar{z}\left\langle\left\{\bar{Q}_{+}, \cdots \prod_{i} \mathcal{O}_{i}\left(z_{i}\right)\right\}\right\rangle=0$. Thus, the correlation functions are always holomorphic in $z$. Secondly, $T_{z \bar{z}}=\left\{\bar{Q}_{+}, G_{z \bar{z}}\right\}$ for some $G_{z \bar{z}}$ in the 'massive' models. Hence, the variation of the correlation functions due to a change in the scale of $\Sigma$ will be given by $\left\langle\mathcal{O}_{1}\left(z_{1}\right) \mathcal{O}_{2}\left(z_{2}\right) \ldots \mathcal{O}_{s}\left(z_{s}\right)\left\{\bar{Q}_{+}, G_{z \bar{z}}\right\}\right\rangle=\left\langle\left\{\bar{Q}_{+}, \prod_{i} \mathcal{O}_{i}\left(z_{i}\right) \cdot G_{z \bar{z}}\right\}\right\rangle=$ 0 . In other words, the correlation functions of local physical operators will continue to be invariant under arbitrary scalings of $\Sigma$. Thus, the correlation functions are always independent of the Kähler structure on $\Sigma$ and depend only on its complex structure.

\section{A Holomorphic Chiral Algebra $\mathcal{A}$}

Let $\mathcal{O}(z)$ and $\widetilde{\mathcal{O}}\left(z^{\prime}\right)$ be two $\bar{Q}_{+}$-closed operators such that their product is $\bar{Q}_{+}$-closed as well. Now, consider their operator product expansion or OPE:

$$
\mathcal{O}(z) \widetilde{\mathcal{O}}\left(z^{\prime}\right) \sim \sum_{k} f_{k}\left(z-z^{\prime}\right) \mathcal{O}_{k}\left(z^{\prime}\right)
$$

in which the explicit form of the coefficients $f_{k}$ must be such that the scaling dimensions and $U(1)_{R}$ charges of the operators agree on both sides of the OPE. In general, $f_{k}$ is not holomorphic in $z$. However, if we work modulo $\bar{Q}_{+}$-exact operators in passing to the $\bar{Q}_{+}{ }^{-}$ cohomology, the $f_{k}$ 's which are non-holomorphic and are thus not annihilated by $\partial / \partial \bar{z}$, drop out from the OPE because they multiply operators $\mathcal{O}_{k}$ which are $\bar{Q}_{+}$-exact. This is true because $\partial / \partial \bar{z}$ acts on the LHS of (A.20) to give terms which are cohomologically trivial 20 In other words, we can take the $f_{k}$ 's to be holomorphic coefficients in studying the $\bar{Q}_{+}{ }^{-}$ cohomology. Thus, the OPE of (A.20) has a holomorphic structure.

In summary, we have established that the $\bar{Q}_{+}$-cohomology of holomorphic local operators has a natural structure of a holomorphic chiral algebra (as defined in the mathematical literature) which we shall henceforth call $\mathcal{A}$; it is always preserved under global translations and dilatations, though (unlike the usual physical notion of a chiral algebra) it may not be preserved under general holomorphic coordinate transformations on the Riemann surface $\Sigma$ depending on whether $c_{1}(X)$ vanishes or not. Likewise, the OPEs of the chiral algebra of local operators obey the usual relations of holomorphy, associativity, and invariance under translations and scalings of $z$, but not necessarily invariance under arbitrary holomorphic reparameterisations of $z$. The local operators are of dimension $(\mathrm{n}, 0)$ for $n \geq 0$, and the chiral

\footnotetext{
${ }^{20}$ Since $\left\{\bar{Q}_{+}, \mathcal{O}\right\}=0$, we have $\partial_{\bar{z}} \mathcal{O}=\left\{\bar{Q}_{+}, V(z)\right\}$ for some $V(z)$, as argued before. Hence $\partial_{\bar{z}} \mathcal{O}(z) \cdot \widetilde{\mathcal{O}}\left(z^{\prime}\right)=$ $\left\{\bar{Q}_{+}, V(z) \widetilde{\mathcal{O}}\left(z^{\prime}\right)\right\}$.
} 
algebra of such operators requires a flat metric up to scaling on $\Sigma$ to be defined. Therefore, the chiral algebra that we have obtained can either be globally-defined on a $\Sigma$ of genus-one, or locally-defined on an arbitrary but curved $\Sigma$. The sigma-model is also plagued by anomalies of the form $\frac{1}{2} c_{1}(\Sigma) c_{1}(X)$ and $\frac{1}{2} p_{1}(X)$, where $p_{1}(X)$ is the first Pontryagin class of $T X$. However, for the flag manifolds $X$ considered in this paper, $p_{1}(X)$ vanishes. In addition, since the chiral algebra that we will be analysing depends only on a local coordinate $z$ on $\Sigma$, i.e., we will only be working locally on $\Sigma$, the first anomaly will also be irrelevant in our context. Therefore, we shall henceforth have nothing more to say about these anomalies. Last but not least, as is familiar for chiral algebras, the correlation functions of these operators depend on $\Sigma$ only via its complex structure. The correlation functions are holomorphic in the parameters of the theory and are therefore protected from perturbative corrections.

\section{A.4. Local Operators as Perturbative Observables}

\section{Local Operators}

In general, a local operator is an operator $\mathcal{F}$ that is a function of the physical fields $\phi^{i}$, $\phi^{\bar{i}}, \psi_{\bar{z}}^{i}, \psi^{\bar{i}}$, and their derivatives with respect to $z$ and $\bar{z} 21$ However, as we saw in $\S$ A.3, the $\bar{Q}_{+}$-cohomology vanishes for operators of dimension $(n, m)$ with $m \neq 0$. Since $\psi_{\bar{z}}^{i}$ and the derivative $\partial_{\bar{z}}$ both have $m=1$ (and recall from $\S$ A.3 that a physical operator cannot have negative $m$ or $n), \bar{Q}_{+}$-cohomology classes can be constructed from just $\phi^{i}, \phi^{\bar{i}}, \psi^{\bar{i}}$ and their derivatives with respect to $z$. Note that the equation of motion for $\psi^{\bar{i}}$ is $D_{z} \psi^{\bar{i}}=0$. Thus, we can ignore the $z$-derivatives of $\psi^{\bar{i}}$, since it can be expressed in terms of the other fields and their corresponding derivatives. Therefore, a chiral (i.e. $\bar{Q}_{+}$-invariant) operator which represents a $\bar{Q}_{+}$-cohomology class is given by $\mathcal{F}\left(\phi^{i}, \partial_{z} \phi^{i}, \partial_{z}^{2} \phi^{i}, \ldots ; \phi^{\bar{i}}, \partial_{z} \phi^{\bar{i}}, \partial_{z}^{2} \phi^{\bar{i}}, \ldots ; \psi^{\bar{i}}\right)$, where we have tried to indicate that $\mathcal{F}$ might depend on $z$ derivatives of $\phi^{i}$ and $\phi^{\bar{i}}$ of arbitrarily high order, though not on derivatives of $\psi^{\bar{i}}$. If the scaling dimension of $\mathcal{F}$ is bounded, it will mean that $\mathcal{F}$ depends only on the derivatives of fields up to some finite order or is a polynomial of bounded degree in those. Notice that $\mathcal{F}$ will always be a polynomial of finite degree in $\psi^{\bar{i}}$, simply because $\psi^{\bar{i}}$ is fermionic and can only have a finite number of components before they vanish due to their anticommutativity. However, the dependence of $\mathcal{F}$ on $\phi^{i}, \phi^{\bar{i}}$ (as opposed to their derivatives) need not have any simple form. Nevertheless, we can make the following observation - from the $U(1)_{R}$ charges of the fields listed in $\S$ A.2, we see that if

\footnotetext{
${ }^{21}$ Note here that since we are interested in local operators which define a holomorphic chiral algebra on the Riemann surface $\Sigma$, we will work locally on $\Sigma$ with local parameter $z$. Hence, we need not include in our operators the dependence on the scalar curvature of $\Sigma$.
} 
$\mathcal{F}$ is homogeneous of degree $k$ in $\psi^{\bar{i}}$, then it has $U(1)_{R^{-c h a r g e}} q_{R}=k$.

A general $q_{R}=k$ operator $\mathcal{F}\left(\phi^{i}, \partial_{z} \phi^{i}, \ldots ; \phi^{\bar{i}}, \partial_{z} \phi^{\bar{i}}, \ldots ; \psi^{\bar{i}}\right)$ can be interpreted as a $(0, k)$-form on $X$ with values in a certain tensor product bundle. In order to illustrate the general idea behind this interpretation, we will make things explicit for operators of dimension $(0,0)$ and $(1,0)$. Similar arguments will likewise apply for operators of higher dimension. For dimension $(0,0)$, the most general operator takes the form $\mathcal{F}\left(\phi^{i}, \phi^{\bar{i}} ; \psi^{\bar{j}}\right)=$ $f_{\bar{j}_{1}, \ldots, \bar{j}_{k}}\left(\phi^{i}, \phi^{\bar{i}}\right) \psi^{\bar{j}_{i}} \ldots \psi^{\bar{j}_{k}}$; thus, $\mathcal{F}$ may depend on $\phi^{i}$, and $\phi^{\bar{i}}$, but not on their derivatives, and is $k^{\text {th }}$ order in $\psi^{\bar{j}}$. Mapping $\psi^{\bar{j}}$ to $d \phi^{\bar{j}}$ (which one can do so as both $\psi^{\bar{j}}$ and $d \phi^{\bar{j}}$ are anticommuting quantities), such an operator corresponds to an ordinary $(0, k)$-form $f_{\bar{j}_{1}, \ldots, \bar{j}_{k}}\left(\phi^{i}, \phi^{\bar{i}}\right) d \phi^{\bar{j}_{1}} \ldots d \phi^{\bar{j}_{k}}$ on $X$. For dimension $(1,0)$, there are two general cases. In the first case, we have an operator $\mathcal{F}\left(\phi^{l}, \partial_{z} \phi^{i} ; \phi^{\bar{l}} ; \psi^{\bar{j}}\right)=f_{i, \bar{j}_{1}, \ldots, \bar{j}_{k}}\left(\phi^{l}, \phi^{\bar{l}}\right) \partial_{z} \phi^{i} \psi^{\bar{j}_{1}} \ldots \psi^{\bar{j}_{k}}$ that is linear in $\partial_{z} \phi^{i}$ and does not depend on any other derivatives. It is a $(0, k)$-form on $X$ with values in the bundle $T^{*} X$; alternatively, it is a $(1, k)$-form on $X$. Similarly, in the second case, we have an operator $\mathcal{F}\left(\phi^{l} ; \phi^{\bar{l}}, \partial_{z} \phi^{\bar{s}} ; \psi^{\bar{j}}\right)=f_{\bar{j}_{1}, \ldots, \bar{j}_{k}}\left(\phi^{l}, \phi^{\bar{l}}\right) g_{i \bar{s}} \partial_{z} \phi^{\bar{s}} \psi^{\bar{j}_{i}} \ldots \psi^{\bar{j}_{k}}$ that is linear in $\partial_{z} \phi^{\bar{s}}$ and does not depend on any other derivatives. It is a $(0, k)$-form on $X$ with values in the bundle $T X$. In a similar fashion, for any integer $n>0$, the operators of dimension $(n, 0)$ and charge $q_{R}=k$ can be interpreted as $(0, k)$-forms with values in a certain bundle over $X$. This structure persists in quantum perturbation theory, but there may be perturbative corrections to the complex structure of the bundle.

The Quantum Action of $\bar{Q}_{+}$

The action of $\bar{Q}_{+}$on such operators can be easily described at the classical level. If we interpret $\psi^{\bar{i}}$ as $d \phi^{\bar{i}}$, then $\bar{Q}_{+}$acts on functions of $\phi^{i}$ and $\phi^{\bar{i}}$, and is simply the $\bar{\partial}$ operator on $X$. This follows from the transformation laws $\delta \phi^{\bar{i}}=\psi^{\bar{i}}, \delta \phi^{i}=0, \delta \psi^{\bar{i}}=0$. The interpretation of $\bar{Q}_{+}$as the $\bar{\partial}$ operator will remain valid when $\bar{Q}_{+}$acts on a more general operator $\mathcal{F}\left(\phi^{i}, \partial_{z} \phi^{i}, \ldots ; \phi^{\bar{i}}, \partial_{z} \phi^{\bar{i}}, \ldots ; \psi^{\bar{i}}\right)$ that does depend on the derivatives of $\phi^{i}$ and $\phi^{\bar{i}}$. The reason for this is because we have the equation of motion $D_{z} \psi^{\bar{i}}=0$. This means that one can neglect the action of $\bar{Q}_{+}$on derivatives $\partial_{z}^{m} \phi^{\bar{i}}$ with $m>0$.

Perturbatively however, there will be corrections to the action of $\bar{Q}_{+}$. In fact, as briefly mentioned in $\S$ A.3 earlier, (A.19) provides such an example - the holomorphic stress tensor $T_{z z}$, though not corrected at 1-loop, is no longer $\bar{Q}_{+}$-closed because the action of $\bar{Q}_{+}$has received perturbative corrections. The fact that $\bar{Q}_{+}$does not always act as the $\bar{\partial}$ operator at the quantum level suggests that one needs a more general framework than just ordinary Dolbeault or $\bar{\partial}$-cohomology to describe the $\bar{Q}_{+}$-cohomology of the twisted $(0,2)$ sigma-model. Indeed, as we will show shortly, the appropriate description of the $\bar{Q}_{+}$-cohomology of local 
operators spanning the chiral algebra will be given in terms of the more abstract notion of Cech cohomology.

\section{A.5. A Sheaf of Chiral Algebras}

We shall now explain the idea of a "sheaf of chiral algebras" on $X$. To this end, note that both the $\bar{Q}_{+}$-cohomology of local operators (i.e., operators that are local on the Riemann surface $\Sigma$ ), and the fermionic symmetry generator $\bar{Q}_{+}$, can be described locally on $X$. Hence, one is free to restrict the local operators to be well-defined not throughout $X$, but only on a given open set $U \subset X$. Since in perturbation theory, we are considering trivial maps $\Phi: \Sigma \rightarrow X$ with no multiplicities, an operator defined in an open set $U$ will have a sensible operator product expansion with another operator defined in $U$. From here, one can naturally proceed to restrict the definition of the operators to smaller open sets, such that a global definition of the operators can be obtained by gluing together the open sets on their unions and intersections. From this description, in which one associates a chiral algebra, its OPEs, and chiral ring to every open set $U \subset X$, we get what is known mathematically as a "sheaf of chiral algebras". We shall call this sheaf $\widehat{\mathcal{A}}$.

\section{Description of $\mathcal{A}$ via Cech Cohomology}

In perturbation theory, one can also describe the $\bar{Q}_{+}$-cohomology classes by a form of Cech cohomology. This alternative description will take us to the mathematical point of view on the subject [11, 32]. In essence, we will show that the chiral algebra $\mathcal{A}$ of the $\bar{Q}_{+}$-cohomology classses of the twisted $(0,2)$ sigma-model on $X$, can be represented, in perturbation theory, by the classes of the Cech cohomology of the sheaf $\widehat{\mathcal{A}}$ of locallydefined chiral operators. To this end, we shall demonstrate an isomorphism between the $\bar{Q}_{+}$-cohomology classes and the classes of the Cech cohomology of $\widehat{\mathcal{A}}$.

Let us start by considering an open set $U \subset X$ that is isomorphic to a contractible space such as an open ball in $\mathbb{C}^{n}$, where $n=\operatorname{dim}_{\mathbb{C}}(X)$. Because $U$ is a contractible space, any bundle over $U$ will be trivial. In the absence of perturbative corrections at the classical level, any operator $\mathcal{F}$ in the $\bar{Q}_{+}$-cohomology will be classes of $H_{\bar{\partial}}^{0, k}(U, \widehat{F})$ on $U$ as explained earlier. Since $\widehat{F}$ will be a trivial bundle over $U$, which means that $\widehat{F}$ will always possess a global section, i.e., it corresponds to a soft sheaf, and because the higher Cech cohomologies of a soft sheaf are trivial [33], we will have $H_{\mathrm{Cech}}^{k}(U, \widehat{F})=0$ for $k>0$. Mapping this back to Dolbeault cohomology via the Cech-Dolbeault isomorphism, we find that $H_{\bar{\partial}}^{0, k}(U, \widehat{F})=0$ for $k>0$. Note that small quantum corrections in the perturbative limit can only annihilate 
cohomology classes and not create them. Hence, in perturbation theory, it follows that the local operators $\mathcal{F}$ with positive values of $q_{R}$, must vanish in $\bar{Q}_{+}$-cohomology on $U$.

Now consider a good cover of $X$ by open sets $\left\{U_{a}\right\}$. Since the intersection of open sets $\left\{U_{a}\right\}$ also give open sets (isomorphic to open balls in $\mathbb{C}^{n}$ ), $\left\{U_{a}\right\}$ and all of their intersections have the same property as $U$ described above: $\bar{\partial}$-cohomology and hence $\bar{Q}_{+}$-cohomology vanishes for positive values of $q_{R}$ on $\left\{U_{a}\right\}$ and their intersections.

Let the operator $\mathcal{F}_{1}$ on $X$ be a $\bar{Q}_{+}$-cohomology class with $q_{R}=1$. It is here that we shall import the usual arguments relating a $\bar{\partial}$ and Cech cohomology, to demonstrate an isomorphism between the $\bar{Q}_{+}$-cohomology and a Cech cohomology. When restricted to an open set $U_{a}$, the operator $\mathcal{F}_{1}$ must be trivial in $\bar{Q}_{+}$-cohomology, i.e., $\mathcal{F}_{1}=\left\{\bar{Q}_{+}, \mathcal{C}_{a}\right\}$, where $\mathcal{C}_{a}$ is an operator of $q_{R}=0$ that is well-defined in $U_{a}$.

Now, since $\bar{Q}_{+}$-cohomology classes such as $\mathcal{F}_{1}$ can be globally-defined on $X$, we have $\mathcal{F}_{1}=\left\{\bar{Q}_{+}, \mathcal{C}_{a}\right\}=\left\{\bar{Q}_{+}, \mathcal{C}_{b}\right\}$ over the intersection $U_{a} \cap U_{b}$, so $\left\{\bar{Q}_{+}, \mathcal{C}_{a}-\mathcal{C}_{b}\right\}=0$. Let $\mathcal{C}_{a b}=$ $\mathcal{C}_{a}-\mathcal{C}_{b}$. For each $a$ and $b, \mathcal{C}_{a b}$ is defined in $U_{a} \cap U_{b}$. Therefore, for all $a, b, c$, we have

$$
\mathcal{C}_{a b}=-\mathcal{C}_{b a}, \quad \mathcal{C}_{a b}+\mathcal{C}_{b c}+\mathcal{C}_{c a}=0
$$

Moreover, for $\left(q_{R}=0\right)$ operators $\mathcal{K}_{a}$ and $\mathcal{K}_{b}$, whereby $\left\{\bar{Q}_{+}, \mathcal{K}_{a}\right\}=\left\{\bar{Q}_{+}, \mathcal{K}_{b}\right\}=0$, we have an equivalence relation

$$
\mathcal{C}_{a b} \sim \mathcal{C}^{\prime}{ }_{a b}=\mathcal{C}_{a b}+\mathcal{K}_{a}-\mathcal{K}_{b}
$$

Note that the collection $\left\{C_{a b}\right\}$ are operators in the $\bar{Q}_{+}$-cohomology with well-defined operator product expansions.

Since the local operators with positive values of $q_{R}$ vanish in $\bar{Q}_{+}$-cohomology on an arbitrary open set $U$, the sheaf $\widehat{\mathcal{A}}$ of the chiral algebra of operators has for its local sections the $\psi^{\bar{i}}$-independent (i.e. $\left.q_{R}=0\right)$ operators $\widehat{\mathcal{F}}\left(\phi^{i}, \partial_{z} \phi^{i}, \ldots ; \phi^{\bar{i}}, \partial_{z} \phi^{\bar{i}}, \ldots\right)$ that are annihilated by $\bar{Q}_{+}$. Each $C_{a b}$ with $q_{R}=0$ is thus a section of $\widehat{\mathcal{A}}$ over the intersection $U_{a} \cap U_{b}$. From (A.21) and (A.22), we find that the collection $\left\{C_{a b}\right\}$ defines the elements of the first Cech cohomology group $H_{\text {Cech }}^{1}(X, \widehat{\mathcal{A}})$.

Next, note that the $\bar{Q}_{+}$-cohomology classes are defined as those operators which are $\bar{Q}_{+}$-closed, modulo those which can be globally written as $\left\{\bar{Q}_{+}, \ldots\right\}$ on $X$. In other words, $\mathcal{F}_{1}$ vanishes in $\bar{Q}_{+}$-cohomology if we can write it as $\mathcal{F}_{1}=\left\{\bar{Q}_{+}, \mathcal{C}_{a}\right\}=\left\{\bar{Q}_{+}, \mathcal{C}_{b}\right\}=\left\{\bar{Q}_{+}, \mathcal{C}\right\}$, i.e., $\mathcal{C}_{a}=\mathcal{C}_{b}$ and hence $\mathcal{C}_{a b}=0$. Therefore, a vanishing $\bar{Q}_{+}$-cohomology with $q_{R}=1$ corresponds to a vanishing first Cech cohomology. Thus, we have obtained a map between the $\bar{Q}_{+}$-cohomology with $q_{R}=1$ and a first Cech cohomology. Similar to the case of relating 
a $\bar{\partial}$ and Cech cohomology, one can also run everything backwards and construct an inverse of this map [10]. Since there is nothing unique about the $q_{R}=1$ case, we can repeat the above procedure for operators with $q_{R}>1$. In doing so, we find that the $\bar{Q}_{+}$-cohomology

coincides with the Cech cohomology of $\widehat{\mathcal{A}}$ for all $q_{R}$. Hence, the chiral algebra $\mathcal{A}$ of the twisted $(0,2)$ sigma-model will be given by $\bigoplus_{q_{R}} H_{\text {Cech }}^{q_{R}}(X, \widehat{\mathcal{A}})$ as a vector space. As there will be no ambiguity, we shall henceforth omit the label "Cech" when referring to the cohomology of $\widehat{\mathcal{A}}$.

Note that in the mathematical literature, the sheaf $\widehat{\mathcal{A}}$, also known as a sheaf of vertex algebras, is studied purely from the Cech viewpoint; the field $\psi^{\bar{i}}$ is omitted and locally on $X$, one considers operators constructed only from $\phi^{i}, \phi^{\bar{i}}$ and their $z$-derivatives. The chiral algebra $\mathcal{A}$ of $\bar{Q}_{+}$-cohomology classes with positive $q_{R}$ are correspondingly constructed as Cech $q_{R}$-cocycles. However, in the physical description via a Lagrangian and $\bar{Q}_{+}$operator, the sheaf $\widehat{\mathcal{A}}$ and its cohomology are given a $\bar{\partial}$-like description, where Cech $q_{R^{-} \text {-cycles are }}$ represented by operators that are $q_{R}^{t h}$ order in the field $\psi^{\bar{i}}$. Notice that the mathematical description does not involve any form of perturbation theory at all. Instead, it utilises the abstraction of Cech cohomology to define the spectrum of operators in the quantum sigmamodel. It is in this sense that the study of the sigma-model is given a rigorous foundation in the mathematical literature.

\section{A.6. Relation to a Free $\beta \gamma$ System}

Now, we shall express in a physical language a few key points that are made in the mathematical literature [32] starting from a Cech viewpoint. Let us start by providing a convenient description of the local structure of the sheaf $\widehat{\mathcal{A}}$. To this end, we will describe in a new way the $\bar{Q}_{+}$-cohomology of operators that are regular in a small open set $U \subset X$. We assume that $U$ is isomorphic to an open ball in $\mathbb{C}^{n}$ and is thus contractible.

Notice from $S_{\text {pert }}$ in (A.13) and $V$ in (A.10), that the hermitian metric on $X$ only appears inside a term of the form $\left\{\bar{Q}_{+}, \ldots\right\}$ in the action. Thus, any shift in the metrics will also appear inside $\bar{Q}_{+}$-exact (i.e. $\bar{Q}_{+}$-trivial) terms. Consequently, for our present purposes, we can arbitrarily redefine the value of the hermitian metric on $X$, since it does not affect the analysis of the $\bar{Q}_{+}$-cohomology. Therefore, to describe the local structure, we can pick a hermitian metric that is flat when restricted to $U$. Thus, the local action (derived from the 
flat hermitian metric) of the twisted $(0,2)$ sigma-model on $U$ is

$$
I=\frac{1}{2 \pi} \int_{\Sigma}\left|d^{2} z\right| \sum_{i, \bar{j}} \delta_{i \bar{j}}\left(\partial_{z} \phi^{\bar{j}} \partial_{\bar{z}} \phi^{i}+\psi_{\bar{z}}^{i} \partial_{z} \psi^{\bar{j}}\right)
$$

Now let us describe the $\bar{Q}_{+}$-cohomology classes of operators regular in $U$. As explained earlier, these are operators of dimension $(n, 0)$ that are independent of $\psi^{\bar{i}}$. In general, such operators are of the form $\widehat{\mathcal{F}}\left(\phi^{i}, \partial_{z} \phi^{i}, \ldots ; \phi^{\bar{i}}, \partial_{z} \phi^{\bar{i}}, \ldots\right)$. Recall that $\bar{Q}_{+}$will act as the $\bar{\partial}$ operator at the classical level. Because perturbative corrections to the action of $\bar{Q}_{+}$can be ignored on a flat open set $U$ [10], on the classes of operators in $U, \bar{Q}_{+}$will continue to act as $\bar{\partial}=\psi^{\bar{i}} \partial / \partial \phi^{\bar{i}}$, and the condition that $\widehat{\mathcal{F}}$ is annihilated by $\bar{Q}_{+}$is precisely that, as a function of $\phi^{i}, \phi^{\bar{i}}$, and their $z$-derivatives, it is independent of $\phi^{\bar{i}}$ (as opposed to its derivatives), and depends only on the other variables, namely $\phi^{i}$, and the derivatives of $\phi^{i}$ and $\phi^{\bar{i}}$.22 Hence, the $\bar{Q}_{+}$-invariant operators are of the form $\widehat{\mathcal{F}}\left(\phi^{i}, \partial_{z} \phi^{i}, \ldots ; \partial_{z} \phi^{\bar{i}}, \partial_{z}^{2} \phi^{\bar{i}}, \ldots\right)$. In other words, the operators, in their dependence on the center of mass coordinate of the string whose worldsheet theory is the twisted $(0,2)$ sigma-model, is holomorphic. The local sections of $\widehat{\mathcal{A}}$ are just given by the operators in the $\bar{Q}_{+}$-cohomology of the local, twisted $(0,2)$ sigma-model with action (A.23).

Let us set $\beta_{i}=\delta_{i \bar{j}} \partial_{z} \phi^{\bar{j}}$ and $\gamma^{i}=\phi^{i}$, whereby $\beta_{i}$ and $\gamma^{i}$ are bosonic operators of dimension $(1,0)$ and $(0,0)$ respectively. Then, the $\bar{Q}_{+}$-cohomology of operators regular in $U$ can be represented by arbitrary local functions of $\beta$ and $\gamma$ of the form $\widehat{\mathcal{F}}\left(\gamma, \partial_{z} \gamma, \partial_{z}^{2} \gamma, \ldots, \beta, \partial_{z} \beta, \partial_{z}^{2} \beta, \ldots\right)$. The operators $\beta$ and $\gamma$ have the operator products of a standard $\beta \gamma$ system. The products $\beta \cdot \beta$ and $\gamma \cdot \gamma$ are non-singular, while

$$
\beta_{i}(z) \gamma^{j}\left(z^{\prime}\right)=-\frac{\delta_{i j}}{z-z^{\prime}}+\text { regular. }
$$

These statements can be deduced from the flat action (A.23) by standard field-theoretic methods. We can write down an action for the fields $\beta$ and $\gamma$, regarded as free elementary fields, which reproduces these OPE's. It is simply the following action of a $\beta \gamma$ system:

$$
I_{\beta \gamma}=\frac{1}{2 \pi} \int\left|d^{2} z\right| \sum_{i} \beta_{i} \partial_{\bar{z}} \gamma^{i}
$$

Hence, we find that the local $\beta \gamma$ system above reproduces the $\bar{Q}_{+}$-cohomology of $\psi^{\bar{i}}$-independent operators of the sigma-model on $U$, i.e., the local sections of the sheaf $\widehat{\mathcal{A}}$.

\footnotetext{
${ }^{22}$ We can again ignore the action of $\bar{Q}_{+}$on $z$-derivatives of $\phi^{\bar{i}}$ because of the equation of motion $\partial_{z} \psi^{\bar{i}}=0$ and the symmetry transformation law $\delta \phi^{\bar{i}}=\psi^{\bar{i}}$.
} 
At this juncture, one can make another important observation concerning the relationship between the local twisted $(0,2)$ sigma-model with action (A.23) and the local version of the $\beta \gamma$ system of (A.25). To begin with, note that the holomorphic stress tensor $\widehat{T}(z)=-2 \pi T_{z z}$ of the local sigma-model is given by

$$
\widehat{T}(z)=-\delta_{i \bar{j}} \partial_{z} \phi^{\bar{j}} \partial_{z} \phi^{i}
$$

(Here and below, normal ordering is understood for $\widehat{T}(z)$ ). Via the respective identification of the fields $\beta$ and $\gamma$ with $\partial_{z} \phi$ and $\phi$, we find that $\widehat{T}(z)$ can be written in terms of the $\beta$ and $\gamma$ fields as

$$
\widehat{T}(z)=-\beta_{i} \partial_{z} \gamma^{i}
$$

$\widehat{T}(z)$, as given by (A.27), coincides with the holomorphic stress tensor of the local $\beta \gamma$ system. Simply put, the twisted $(0,2)$ sigma-model and the $\beta \gamma$ system have the same local holomorphic stress tensor. This means that locally on $X$, the sigma-model and the $\beta \gamma$ system have the same generators of general holomorphic coordinate transformations on the worldsheet.

One may now ask the following question: does the $\beta \gamma$ system reproduce the $\bar{Q}_{+}{ }^{-}$ cohomology of $\psi^{\bar{i}}$-independent operators globally on $X$, or only in a small open set $U$ ? Well, the $\beta \gamma$ system will certainly reproduce the $\bar{Q}_{+}$-cohomology of $\psi^{\bar{i}}$-independent operators globally on $X$ if there is no obstruction to defining the system globally on $X$, i.e., one finds, after making global sense of the action (A.25), that the corresponding theory remains anomaly-free. Let's look at this more closely.

First and foremost, the classical action (A.25) makes sense globally if we interpret the bosonic fields $\beta$, $\gamma$ correctly. $\gamma$ defines a map $\gamma: \Sigma \rightarrow X$, and $\beta$ is a $(1,0)$-form on $\Sigma$ with values in the pull-back $\gamma^{*}\left(T^{*} X\right)$. With this interpretation, (A.25) becomes the action of what one might call a non-linear $\beta \gamma$ system. However, by choosing $\gamma^{i}$ to be local coordinates on a small open set $U \subset X$, one can make the action linear. In other words, a local version of (A.25) represents the action of a linear $\beta \gamma$ system.

Now that we have made global sense of the action of the $\beta \gamma$ system at the classical level, we move on to discuss what happens at the quantum level. The anomalies that enter in the twisted $(0,2)$ sigma model also appear in the nonlinear $\beta \gamma$ system as follows. Expand around a classical solution of the nonlinear $\beta \gamma$ system, represented by a holomorphic map $\gamma_{0}: \Sigma \rightarrow X$. Setting $\gamma=\gamma_{0}+\gamma^{\prime}$, the action, expanded to quadratic order about this solution, is $(1 / 2 \pi)\left(\beta, \bar{D} \gamma^{\prime}\right) \cdot \gamma^{\prime}$, being a deformation of the coordinate $\gamma_{0}$ on $X$, is a section of the pull-back $\gamma_{0}^{*}(T X)$. Thus, the kinetic operator of the $\beta$ and $\gamma$ fields is the $\bar{D}$ operator 
on sections of $\gamma_{0}^{*}(T X)$; it is the complex conjugate of the $D$ operator of the fermion kinetic term of the twisted sigma-model action $S_{\text {pert }}$ that results in its observed anomalies. Complex conjugation reverses the sign of the anomalies, but here the fields are bosonic, while in the twisted sigma-model, they are fermionic; this gives a second sign change.23 Hence, the non-linear $\beta \gamma$ system has exactly the same anomalies as the underlying twisted $(0,2)$ sigmamodel. And if the anomalies vanish, the $\beta \gamma$ system will reproduce the $\bar{Q}_{+}$-cohomology of $\psi^{\bar{i}}$-independent operators globally on $X$. In other words, one can find a global section of $\widehat{\mathcal{A}}$ in such a case.

However, note that the $\beta \gamma$ system lacks the presence of right-moving fermions and thus the $U(1)_{R}$ charge $q_{R}$ carried by the fields $\psi_{\bar{z}}^{i}$ and $\psi^{\bar{i}}$ of the underlying twisted $(0,2)$ sigmamodel. Locally, the $\bar{Q}_{+}$-cohomology of the sigma model is non-vanishing only for $q_{R}=0$. Globally however, there can generically be cohomology in higher degrees. Since the chiral algebra of operators furnished by the linear $\beta \gamma$ system gives the correct description of the $\bar{Q}_{+}$-cohomology of $\psi^{\bar{i}}$-independent operators on $U$, one can then expect the globally-defined chiral algebra of operators furnished by the non-linear $\beta \gamma$ system to correctly describe the $\bar{Q}_{+}$-cohomology classes of zero degree (i.e. $q_{R}=0$ ) on $X$. How then can one use the nonlinear $\beta \gamma$ system to describe the higher cohomology? The answer lies in the analysis carried out in the $\S$ A.5. In the $\beta \gamma$ description, we do not have a close analog of $\bar{\partial}$ cohomology at our convenience. Nevertheless, we can use the more abstract notion of Cech cohomology. As before, we begin with a good cover of $X$ by small open sets $\left\{U_{a}\right\}$, and, as explained in $\S$ A.5, we can then describe the $\bar{Q}_{+}$-cohomology classes of positive degree (i.e. $q_{R}>0$ ) by Cech $q_{R}$-cocycles, i.e., they can be described by the $q_{R}^{\text {th }}$ Cech cohomology of the sheaf $\widehat{\mathcal{A}}$ of the chiral algebra of the linear $\beta \gamma$ system with action being a linearised version of (A.25). Although unusual from a physicist's perspective, this Cech cohomology approach has been taken as a starting point for the present subject in the mathematical literature [11, 32].

\section{A.7. Local Symmetries, Gluing the Free $\beta \gamma$ Systems, and the Sheaf of CDO's}

\section{Conserved Currents and Local Symmetries}

So far, we have obtained an understanding of the local structure of the $\bar{Q}_{+}$-cohomology. We shall now proceed towards our real objective of obtaining an understanding of its global structure, since after all, the sigma-model is defined on all of $X$, and not just some open set

\footnotetext{
${ }^{23}$ Notice that the $D$ operator in $S_{\text {pert }}$ acts on sections of the pull-back of the anti-holomorphic bundle $\overline{T X}$ instead of the holomorphic bundle $T X$. However, this difference is irrelevant with regard to anomalies since $p_{1}(\overline{T X})=p_{1}(T X)$.
} 
$U$. In order to do, we will need to glue the local descriptions that we have studied above together, so that we will appropriately have a globally-defined $\beta \gamma$ system and its chiral algebra at our disposal.

To this end, we must first cover $X$ by small open sets $\left\{U_{a}\right\}$. Recall here that in each $U_{a}$, the $\bar{Q}_{+}$-cohomology is described by the chiral algebra of local operators of a free $\beta \gamma$ system on $U_{a}$. Next, we will need to glue these local descriptions together over the intersections $\left\{U_{a} \cap U_{b}\right\}$, so as to describe the global structure of the $\bar{Q}_{+}$-cohomology in terms of a globallydefined sheaf of chiral algebras over the entire manifold $X$.

Note that the gluing has to be carried out using the automorphisms of the free $\beta \gamma$ system. Thus, one must first ascertain the underlying symmetries of the system, which are in turn divided into geometrical and non-geometrical symmetries. The geometrical symmetries are used in gluing together the local sets $\left\{U_{a}\right\}$ into the entire manifold $X$. The non-geometrical symmetries on the other hand, are used in gluing the local descriptions at the algebraic level.

As usual, the generators of these symmetries will be given by the charges of the conserved currents of the free $\beta \gamma$ system. Since the conserved charges must also be conformallyinvariant, it will mean that they must be given by an integral of a dimension one current, modulo total derivatives. The dimension one currents of the free $\beta \gamma$ system can be constructed as follows.

Let us describe the currents which are associated with the geometrical symmetries first. Firstly, if we have a holomorphic vector field $V$ on $X$ where $V=V^{i}(\gamma) \frac{\partial}{\partial \gamma^{2}}$, we can construct a dimension one current $J_{V}=-V^{i} \beta_{i}$. The corresponding conserved charge is then given by $K_{V}=\oint J_{V} d z$. A computation of the operator product expansion with the elementary fields $\gamma$ gives

$$
J_{V}(z) \gamma^{k}\left(z^{\prime}\right) \sim \frac{V^{k}\left(z^{\prime}\right)}{z-z^{\prime}} .
$$

Under the symmetry transformation generated by $K_{V}$, we have $\delta \gamma^{k}=i \epsilon\left[K_{V}, \gamma^{k}\right]$, where $\epsilon$ is a infinitesinal transformation parameter. Thus, we see from (A.28) that $K_{V}$ generates the infinitesimal diffeomorphism $\delta \gamma^{k}=i \epsilon V^{k}$ of $U$. In other words, $K_{V}$ generates the holomorphic diffeomorphisms of the target space $X$. For finite diffeomorphisms, we will have a coordinate transformation $\tilde{\gamma}^{k}=g^{k}(\gamma)$, where each $g^{k}(\gamma)$ is a holomorphic function in the $\gamma^{k}$ s. Since we are using the symmetries of the $\beta \gamma$ system to glue the local descriptions over the intersections $\left\{U_{a} \cap U_{b}\right\}$, on an arbitrary intersection $U_{a} \cap U_{b}, \gamma^{k}$ and $\tilde{\gamma}^{k}$ must be defined in $U_{a}$ and $U_{b}$ respectively. 
We shall now determine the current associated with the non-geometrical symmetries. The charge of the current should not generate any transformations on the $\gamma^{i}$ s at all since these fields have a geometrical interpretation as the coordinates on $X$. In other words, the current must be constructed out of the $\gamma^{i}$ s and their derivatives only. Thus, a suitable dimension one current would be given by $J_{B}=B_{i}(\gamma) \partial_{z} \gamma^{i}$, where the $B_{i}(\gamma)$ 's are just holomorphic functions in the $\gamma^{i}$ s. The conserved charge is then given by $K_{B}=\oint J_{B} d z$. As explained in [10], the $B_{i}(\gamma)$ 's must be the components of an arbitrary holomorphic $(1,0)$ form $B=\sum_{i} B_{i}(\gamma) d \gamma^{i}$ on $X$ that is non-exact, i.e., for every non-vanishing $K_{B}$, there is a $(2,0)$-form $C=\partial B$, that is $\partial$-closed (since $\partial^{2}=0$ ). Thus, $C$ corresponds to a sheaf $\Omega_{X}^{2, c l}$ of $\partial$-closed $(2,0)$-forms on $X$, which is related via the first Cech cohomology group $H^{1}\left(X, \Omega_{X}^{2, c l}\right)$ to the moduli of the chiral algebra of the sigma-model [10, 11]. This point will be important in our forthcoming paper, where we will investigate the physical interpretation of a "quantum" geometric Langlands correspondence in a similar context, albeit with fluxes that correspond to the moduli of the chiral algebra turned on.

\section{Local Field Transformations and Gluing the Free $\beta \gamma$ Systems}

Let us now describe how the different fields of the free $\beta \gamma$ system on any $U$ will transform under the geometrical and non-geometrical symmetries generated by $K_{V}$ and $K_{B}$ respectively. Via a computation of the relevant OPEs, we have

$$
\begin{aligned}
& \tilde{\gamma}^{i}=g^{i}(\gamma) \\
& \tilde{\beta}_{i}=\beta_{k} D_{i}^{k}+\partial_{z} \gamma^{j} E_{i j},
\end{aligned}
$$

where $i, j, k=1,2, \ldots, N=\operatorname{dim}_{\mathbb{C}} X$. Here, $D$ and $E$ are $N \times N$ matrices such that $[D]^{T}=$ $[\partial g]^{-1}$ and $[E]=[\partial B]$, that is, $\left[\left(D^{T}\right)^{-1}\right]_{i}{ }^{k}=\partial_{i} g^{k}$ and $[E]_{i j}=\partial_{i} B_{j}$.

Note that in order to consistently glue a pair of free $\beta \gamma$ systems in any overlap region $U_{a} \cap U_{b}$, one will need to use the relations in (A.29) (A.30) to glue their free fields together. As required, (A.29) - (A.30) defines an automorphism of the free $\beta \gamma$ system - the $\tilde{\gamma}^{i}$ and $\tilde{\beta}^{i}$ fields produce the correct OPE's amongst themselves.

\section{$A$ Sheaf of $C D O$ 's}

Last but not least, note that A.29) - A.30) actually define the automorphism relations of a sheaf $\widehat{\mathcal{O}}_{X}^{c h}$ of Chiral Differential Operators or CDO's on $X[11]$. In other words, $\widehat{A} \simeq \widehat{\mathcal{O}}_{X}^{c h}$. Hence, the $\bar{Q}_{+}$-cohomology and therefore the holomorphic chiral algebra $\mathcal{A}$ of the twisted $(0,2)$ sigma-model will be given by $\bigoplus_{q_{R}} H^{q_{R}}\left(X, \widehat{\mathcal{O}}_{X}^{c h}\right)$ - the sum of all Cech cohomology groups of the sheaf of CDO's on $X$, as a vector space. 


\section{Acknowledgements:}

I'm especially indebted to E. Frenkel for explaining many things in connection with the geometric Langlands program to me over our often tedious email correspondences. I'm also thankful to I. Bakas, D. Ben-Zvi, S. Gukov, F. Malikov and V. Pestun for illuminating exchanges. Last but not least, I would like to thank H. Sati for encouragement, E. Witten for many constructive criticisms, and the JHEP referee for the suggested clarifications. This work is supported by the Institute for Advanced Study and the NUS - Overseas Postdoctoral Fellowship.

\section{References}

[1] A. Kapustin and E. Witten, "Electric-Magnetic Duality and the Geometric Langlands Program", arXiv:hep-th/0604151.

[2] E. Frenkel, "Affine Algebras, Langlands Duality and Bethe Ansatz", arXiv:q-alg/9506003.

[3] E. Frenkel and D. Gaitsgory, "Local Geometric Langlands Correspondence and Affine Kac-Moody Algebras", arXiv:math/0508382.

[4] A. Chervov and D. Talalaev, "Quantum Spectral Curves, Quantum Integrable Systems and the Geometric Langlands Correspondence", arXiv:hep-th/0604128.

[5] A.V. Stoyanovsky, "Quantum Langlands Duality and Conformal Field Theory", arXiv:math/0610974.

[6] E. Frenkel, "Lectures on the Langlands Program and Conformal Field Theory", arXiv:hep-th/0512172.

[7] E. Frenkel and D. Ben-Zvi, "Vertex Algebras and Algebraic Curves", American Mathematical Society, 2001.

[8] B. Feigin and E. Frenkel, "Affine Kac-Moody algebras at the critical level and GelfandDikii algebras", Int. J. Mod. Phys. A7S1A, 197-215.

[9] E. Frenkel, "W-algebras and Langlands -Drinfeld Correspondence", in New Symmetries in Quantum Field Theory, eds. J.Fröhlich, e.a., pp. 437-447, Plenum Press, New York, 1992. 
[10] E. Witten, "Two-Dimensional Models with (0,2) Supersymmetry: Perturbative Aspects", ATMP Vol. 11 - Number 1, arXiv:hep-th/0504078.

[11] F. Malikov, V. Schechtman, and A. Vaintrob, "Chiral de Rham Complex," arXiv:math.AG/9803041.

[12] P. Bouwknegt and K. Schoutens, "W-Symmetry in Conformal Field Theory", Phys.Rept. 223 (1993) 183-276, |arXiv:hep-th/9210010|.

[13] J. Balog, L. Feher, P. Forgacs, L. O'Raifeartaigh and A. Wipf, "Liouville and Toda theories as conformally reduced WZNW theories", Phys. Lett. 227B (1989) 214.

[14] J. Balog, L. Feher, L. O’Raifeartaigh, P. Forgacs and A. Wipf, "Toda theory and Walgebra from a gauged WZNW point of view", Ann. Phys. 203 (1990) 76.

[15] J. Polchinski, "String Theory Vol 2: Superstring Theory and Beyond", (Cambridge Monographs on Mathematical Physics), Cambridge Press.

[16] S.V. Ketov, "Conformal Field Theory", World Scientific Press.

[17] J. Fuchs, "Affine Lie Algebras and Quantum Groups: An Introduction, with Applications in Conformal Field Theory", (Cambridge Monographs on Mathematical Physics), Cambridge Press.

[18] U. Lindström, M. Zabzine, "Tensionless strings, WZW models at critical level and massless higher spin fields", Phys. Lett. 584B, 178-185. arXiv:hep-th/0305098.

[19] I. Bakas, C. Sourdis, "On the tensionless limit of gauged WZW models", JHEP 0406 (2004) 049. arXiv:hep-th/0403165].

[20] M. Bershadsky, H. Ooguri, "Hidden $S L(N)$ symmetry in conformal field theories", Commun. Math. Phys 129 (1989) pp. 49-83.

[21] L. Feher, L. O'raifeartaigh, P. Ruelle, I. Tsutsui, A. Wipf, "On the General Structure of Hamiltonian Reductions of the WZNW Theory", arXiv:hep-th/9112068.

[22] A. Arvanitoyeorgos, "An introduction to Lie groups and the geometry of homogenous spaces", Student Mathematical Library Vol. 22, AMS.

[23] M. Wakimoto, "Fock Space Representations of the Affine Lie Algebra $A_{1}^{(1)}$ ", Commun. Math. Phys. 104 (1986) 609. 
[24] A.M. Polyakov and P.B. Wiegmann, Phys. Lett. 131B (1983) 121.

[25] F.A. Bais and P. Bouwknegt, K. Schoutens and M.Surridge, "Extensions of the Virasoro algebra constructed from Kac-Moody algebras using higher order Casimir invariants", Nucl. Phys. B304 (1988) 348.

[26] Fateev, V., Zamolodchikov, A.: "Conformal quantum field theory models in two dimensions having $Z_{3}$ Symmetry", Nucl. Phys. B248, 551 (1984).

[27] B.L. Feigin and E. Frenkel, "Quantisation of the Drinfeld-Sokolov reduction", Phys. Lett. 246B (1990) 75.

[28] J. de Boer, T. Tjin, "The relation between quantum $\mathcal{W}$-algebras and Lie algebras", Commun. Math. Phys. bf 160, 317 (1994).

[29] P. Di Francesco, P. Mathieu, D. Senechal, "Conformal Field Theory", Springer-Verlag (1999).

[30] T.Eguchi and H.Ooguri, "Conformal and current algebras on a general Riemann surface", Nucl. Phys. B282 (1987) 308-328.

[31] E. Frenkel, "Lectures on Wakimoto modules, opers and the center at the critical level", arXiv:math/0210029.

[32] V. Gorbounov, F. Malikov, and V. Schechtman, Gerbes Of Chiral Differential Operators, [arXiv:math.AG/9906117].

[33] Wells R.O., "Differential Analysis on Complex Manifolds", Springer-Verlag, New York, (1980). 